Article

\title{
The Existence of rG Family and tG Family, and Their Geometric Invariants
}

\author{
Norio Ejiri ${ }^{1, \dagger}$ and Toshihiro Shoda ${ }^{2, *, \ddagger}$ \\ 1 Department of Mathematics, Meijo University, Tempaku, Nagoya 468-8502, Japan; ejiri@meijo-u.ac.jp \\ 2 Faculty of Education, Saga University, 1 Honjo-machi, Saga 840-8502, Japan \\ * Correspondence: tshoda@cc.saga-u.ac.jp \\ $+\quad$ Partly supported by JSPS Grant-in-Aid for Scientific Research (C) 15K04859. \\ $\ddagger \quad$ Partly supported by JSPS Grant-in-Aid for Scientific Research (C) $20 \mathrm{~K} 03616$.
}

Received: 2 September 2020; Accepted: 21 September 2020; Published: 2 October 2020

check for updates

\begin{abstract}
In the 1990s, physicists constructed two one-parameter families of compact oriented embedded minimal surfaces in flat three-tori by using symmetries of space groups, called the $\mathrm{rG}$ family and $\mathrm{tG}$ family. The present work studies the existence of the two families via the period lattices. Moreover, we will consider two kinds of geometric invariants for the two families, namely, the Morse index and the signature of a minimal surface. We show that Schwarz P surface, D surface, Schoen's gyroid, and the Lidinoid belong to a family of minimal surfaces with Morse index 1.
\end{abstract}

Keywords: minimal surfaces; flat tori; Morse index; signature

\section{Introduction}

A triply periodic minimal surface in $\mathbb{R}^{3}$ is concerned with natural phenomena, and it has been studied in physics, chemistry, crystallography, and so on. It can be replaced by a compact oriented minimal surface in a flat three-torus $f: M \rightarrow \mathbb{R}^{3} / \Lambda$ and the conformal structure induced by the immersion $f$ makes $M$ a Riemann surface. We usually call such $f$ a conformal minimal immersion. Applying the complex function theory gives us a useful description of a triply periodic minimal surface. In fact, the following is one of basic tools.

Theorem 1 (Weierstrass representation formula). Let $f: M \rightarrow \mathbb{R}^{3} / \Lambda$ be a conformal minimal immersion. Then, up to translations, $f$ can be represented by the following path-integrals:

$$
f(p)=\Re \int_{p_{0}}^{p} t\left(\omega_{1}, \omega_{2}, \omega_{3}\right) \bmod \Lambda,
$$

where $p_{0}$ is a fixed point on $M$ and the $\omega_{i}$ 's are holomorphic differentials on $M$ satisfying the following three conditions.

$$
\begin{aligned}
& \omega_{1}^{2}+\omega_{2}^{2}+\omega_{3}^{2}=0, \\
& \omega_{1}, \omega_{2}, \omega_{3} \text { have no common zeros, } \\
& \left\{\Re \int_{C}{ }^{t}\left(\omega_{1}, \omega_{2}, \omega_{3}\right) \mid C \in H_{1}(M, \mathbb{Z})\right\} \text { is a sublattice of } \Lambda .
\end{aligned}
$$

Conversely, the real part of path-integrals of holomorphic differentials satisfying the above three conditions defines a conformal minimal immersion. 
In 1970, Schoen [1] discovered a triply periodic minimal surface in $\mathbb{R}^{3}$ which is called the gyroid, and it has been studied in natural sciences. In 1993, Fogden, Haeberlein, and Hyde [2] constructed the $r G$ family and the $t G$ family which are two one-parameter families including the gyroid. They gave their representation formulae as follows.

Example 1 (rG family). For $a=s e^{i \phi}\left(0<s<1, \phi \in\left(-\frac{\pi}{6}, \frac{\pi}{3}\right]\right)$, let $M$ be the hyperelliptic Riemann surface of genus three defined by $w^{2}=z\left(z^{3}-a^{3}\right)\left(z^{3}+\frac{1}{a^{3}}\right)$. Set $f(p)=\Re\left\{e^{i \theta} \int_{p_{0}}^{p}\left(1-z^{2}, i\left(1+z^{2}\right), 2 z\right) \frac{d z}{w}\right\}$. For $(\phi, s, \theta)=\left(\frac{\pi}{3}, 0.494722 \ldots, 0\right), f$ belongs to the rPD family. $f$ must be the Lidinoid for $(\phi, s, \theta)=\left(\frac{\pi}{6}, 0.5361,0.335283 \ldots\right)$. Moreover, $f$ must be the gyroid for $(\phi, s, \theta)=\left(0, \frac{1}{\sqrt{2}}, 0.663483 \ldots\right)$.

Example 2 (tG family). For $b=s e^{i \phi}\left(0<s<1,|\phi|<\frac{\pi}{4}\right)$, let $M$ be the hyperelliptic Riemann surface of genus three defined by $w^{2}=z^{8}+a z^{4}+1=\left(z^{4}+b^{4}\right)\left(z^{4}+\frac{1}{b^{4}}\right)$. Set $f(p)=$ $\Re\left\{i e^{i \theta} \int_{p_{0}}^{p_{t}}\left(1-z^{2}, i\left(1+z^{2}\right), 2 z\right) \frac{d z}{w}\right\}$. For $(\phi, s, \theta)=\left(0, \frac{\sqrt{3}-1}{\sqrt{2}}, 0.663483 \ldots\right), f$ must be the gyroid. $f$ tends to be an element of the $t D$ family as $(\phi, s, \theta) \rightarrow\left(-\frac{\pi}{4}, 0.431882, \frac{\pi}{2}\right)$.

Note that we have to determine $\phi, s, \theta$ such that $f$ is well-defined in Examples 1 and 2 . Fogden, Haeberlein, and Hyde used the computer simulation to show that $s$ and $\theta$ are functions of $\phi$, that is, $s=s(\phi)$ and $\theta=\theta(\phi)$. Hence, the $\mathrm{rG}$ family and the $\mathrm{tG}$ family can be considered as one-parameter families by $\phi$. After that, Fogden and Hyde [3] reconstructed the two families via symmetries of space groups. In this paper, we will consider the existence of them by period calculations and obtain sufficient conditions for it (see Theorems 2 and 3). As their applications, we have numerical evidence that the rG family and the $t G$ family are defined as one-parameter families of compact oriented embedded minimal surfaces of genus three in flat three-tori (Theorem 5).

Next, we will consider the Morse index and the signature of the $\mathrm{rG}$ family and the $\mathrm{tG}$ family. The Morse index (resp. the nullity) of a compact oriented minimal surface in a flat three-torus is defined as the number of negative eigenvalues (resp. zero eigenvalues) of the area, counted with multiplicities. In this case, it is easy to verify that the Morse index is greater than or equal to 1. Ros [4] proved that every compact oriented minimal surface in a flat three-torus with Morse index 1 has genus three. This result suggests that compact oriented minimal surfaces of genus three in flat three-tori are important objects. Recently, the first author has established an algorithm to compute the Morse index and the nullity of a minimal surface (see [5,6], see also [7] for an explanation of the algorithm). By applying the algorithm to the genus three case, the Morse index can be translated into the number of negative eigenvalues of an $18 \times 18$ real symmetric matrix $W_{2}-W_{1}$ defined in Section 3 and the nullity can be translated into the number of zero eigenvalues of a $9 \times 9$ Hermitian matrix $W$ defined in Section 3, counted with multiplicities. The two key matrices consist of periods of the Abelian differentials of the second kind on a minimal surface. We now call a pair of the number of positive eigenvalues and that of negative eigenvalues of $W$, counted with multiplicities the signature of a minimal surface. In the previous paper [8], we carried out the algorithm for five one-parameter families (the $\mathrm{H}$ family, the rPD family, the tP family, the tD family, the tCLP family) and computed their Morse indices and signatures. We will continue this work and give explicit descriptions of $W$ and $W_{2}-W_{1}$ for the $\mathrm{rG}$ family and the $\mathrm{tG}$ family in Section 3. We then numerically compute the Morse indices and the signatures of the rG family and the $\mathrm{tG}$ family (Theorems 7 and 9). Moreover, we can show that there exists a path with the following two properties (Theorem 10): (i) it connects the P surface, the D surface, the gyroid, the Lidinoid ( $\mathrm{G} \rightarrow \mathrm{L} \rightarrow \mathrm{P} \rightarrow \mathrm{D}$ ), (ii) it consists of minimal surfaces with Morse index 1. Recall that this family is called the rGPD union in [3], and our result implies that the rGPD union consists of minimal surfaces with Morse index 1.

The outline of the paper is as follows. In Section 2, we discuss of well-definedness and embeddedness for the $\mathrm{rG}$ family and the $\mathrm{t} G$ family. We shall give sufficient conditions that the rG family and the $\mathrm{tG}$ family are defined as two families of compact oriented minimal surfaces of genus 
three in flat three-tori. After that, we use numerical arguments and show that the two families are defined as one-parameter families of embedded minimal surfaces as in Fogden-Haeberlein-Hyde's paper. Section 3 gives explicit descriptions of the two key matrices to compute the Morse indices and the signatures for the $\mathrm{rG}$ family and the $\mathrm{t} G$ family. Moreover, we shall numerically compute the Morse indices and the signatures of the $\mathrm{rG}$ family and the $\mathrm{t} G$ family. The above two sections need period calculations of the two families and finally in Appendix A there is a collection of the details of period calculations.

All figures except for Figures 6, 9 and 10 and all numerical arguments in Sections 2 and 3 are generated by Mathematica [9].

\section{The Existence and Embeddedness of the rG Family and tG Family}

In this section, we shall give two sufficient conditions that (i) the rG family can be obtained as a deformation from an element of the rPD family, (ii) the $\mathrm{t} G$ family can be obtained as a deformation from the gyroid. As their applications, we can show the existence of the $\mathrm{rG}$ family and the $\mathrm{t} G$ family by numerical arguments. Moreover, we will discuss the embeddedness of the $\mathrm{rG}$ family and the $\mathrm{t} G$ family.

We first review fundamental arguments. Recall that a $3 \times 6$ real matrix $L$ generates a lattice in $\mathbb{R}^{3}$ if and only if there exist a $3 \times 3$ real regular matrix $X$, a $3 \times 6$ matrix $g$ with integer entries, and a $6 \times 3$ matrix $h$ with integer entries such that $L h=X$ and $X g=L$. Thus, we have the following key fact (see $\S 6$ in [5] and $\S 6.2 .2$ in [6]).

Proposition 1. Let $L$ be a $3 \times 6$ real matrix which generates a lattice in $\mathbb{R}^{3}$. Suppose that $L(t)$ is a smooth deformation of $L$ with $L(0)=L$ and $\operatorname{rank}_{\mathbb{R}}(L(t))=3$. Then, $L(t)$ generates a lattice in $\mathbb{R}^{3}$ if and only if there exist a $3 \times 6$ matrix $g$ with integer entries and a $6 \times 3$ matrix $h$ with integer entries such that $g h=E_{3}$ and $L(t) h g=L(t)$.

Next we shall consider the existence of the $\mathrm{rG}$ family and the $\mathrm{t} G$ family by Proposition 1.

\section{1. rG Family}

For $a=s e^{i \phi}\left(0<s<1, \phi \in\left(-\frac{\pi}{6}, \frac{\pi}{3}\right]\right)$, let $M$ be the hyperelliptic Riemann surface of genus three defined by $w^{2}=z\left(z^{3}-a^{3}\right)\left(z^{3}+\frac{1}{a^{3}}\right)$. Define $G_{1}={ }^{t}\left(\frac{1-z^{2}}{w} d z, \frac{i\left(1+z^{2}\right)}{w} d z, \frac{2 z}{w} d z\right)$ as a column vector which consists of a basis of holomorphic differentials on $M$. We now use the notation as in Appendix A.1 of Appendix A. Let $\left\{A_{j}, B_{j}\right\}_{j=1}^{3}$ be the canonical homology basis on $M$ as in Appendix A.1.1 of Appendix A, and set

$$
L=\Re\left\{e^{i \theta}\left(\int_{A_{1}} G_{1} \quad \int_{A_{2}} G_{1} \quad \int_{A_{3}} G_{1} \int_{B_{1}} G_{1} \quad \int_{B_{2}} G_{1} \quad \int_{B_{3}} G_{1}\right)\right\} .
$$

We assume $\theta=0$ for $\phi=\frac{\pi}{3}$. In this case,

$$
\Re \int_{p_{0}}^{p} e^{i \theta} G_{1}
$$

defines a conformal minimal immersion of $M$ into a flat three-torus $\mathbb{R}^{3} / \Lambda$. It belongs to the rPD family and $\Lambda$ is given by

$$
\begin{aligned}
\Lambda= & \left(\begin{array}{ccc}
3 \alpha & 3 \alpha & 2 \alpha \\
\sqrt{3} \alpha & -\sqrt{3} \alpha & 0 \\
0 & 0 & -\beta
\end{array}\right), \\
& \quad\left(\alpha=\frac{1}{s} \int_{0}^{1} \frac{1-s^{2} t^{2}}{\sqrt{t\left(1-t^{3}\right)} \sqrt{s^{3} t^{3}+\frac{1}{s^{3}}}} d t, \beta=4 \int_{0}^{1} \frac{t}{\sqrt{t\left(1-t^{3}\right)} \sqrt{s^{3} t^{3}+\frac{1}{s^{3}}}} d t\right) .
\end{aligned}
$$


In fact, by setting $(Z, W)=\left(e^{\frac{\pi}{3} i} z, e^{\frac{\pi}{6} i} w\right), M$ and $G_{1}$ can be rewritten as

$$
W^{2}=Z\left(Z^{3}-s^{3}\right)\left(Z^{2}+\frac{1}{s^{3}}\right), \quad G_{1}=i\left(\begin{array}{ccc}
-\frac{1}{2} & -\frac{\sqrt{3}}{2} & 0 \\
\frac{\sqrt{3}}{2} & -\frac{1}{2} & 0 \\
0 & 0 & -1
\end{array}\right)\left(\begin{array}{c}
1-Z^{2} \\
i\left(1+Z^{2}\right) \\
2 Z
\end{array}\right) \frac{d Z}{W} .
$$

So it belongs to the $\operatorname{rPD}$ family (see $\S 1$ in [8]). By choosing the branch $\sqrt{a^{3} t^{3}+\frac{1}{a^{3}}}=$ $-i \sqrt{s^{3} t^{3}+\frac{1}{s^{3}}} \in-i \mathbb{R}_{>0}$ and (A12) from Appendix A, we find

$$
L=\left(\begin{array}{cccccc}
-\alpha & 0 & 0 & \alpha & 3 \alpha & 2 \alpha \\
-\sqrt{3} \alpha & 0 & 0 & \sqrt{3} \alpha & \sqrt{3} \alpha & 0 \\
-\beta & 3 \beta & -3 \beta & -2 \beta & 0 & -\beta
\end{array}\right)
$$

for $\phi=\frac{\pi}{3}$. Thus, setting

$$
g=\left(\begin{array}{cccccc}
-1 & 1 & -1 & 0 & 1 & 0 \\
0 & 1 & -1 & -1 & 0 & 0 \\
1 & -3 & 3 & 2 & 0 & 1
\end{array}\right), \quad h=\left(\begin{array}{ccc}
0 & 0 & 0 \\
0 & 0 & 0 \\
0 & 0 & 0 \\
0 & -1 & 0 \\
1 & 0 & 0 \\
0 & 2 & 1
\end{array}\right)
$$

we have $L h=\Lambda, \Lambda g=L$, and $g h=E_{3}$. We now apply Proposition 1 to $L$ given by (1) for $\phi \in\left(-\frac{\pi}{6}, \frac{\pi}{3}\right]$.

Set $L=\left(L_{1}, L_{2}, L_{3}, L_{4}, L_{5}, L_{6}\right)$, where $L_{j}$ is a $3 \times 1$ real matrix. Then, by (A12), we obtain

$$
\begin{aligned}
\operatorname{Lhg}=L & \Longleftrightarrow L_{1}=-L_{5}+L_{6}, L_{2}=-L_{3}=-L_{4}+L_{5}-L_{6} \\
& \Longleftrightarrow \Re\left\{e^{i \theta}(A+\sqrt{3} i B)\right\}=\Re\left\{e^{i \theta}(i C+D)\right\}=0 \\
& \Longleftrightarrow \tan \theta=\frac{\Re(A+\sqrt{3} i B)}{\Im(A+\sqrt{3} i B)}=\frac{\Re(i C+D)}{\Im(i C+D)} .
\end{aligned}
$$

Therefore, we find the following result.

Theorem 2. Let $\phi \in\left(-\frac{\pi}{6}, \frac{\pi}{3}\right]$. Suppose that there exist s and $\phi$ such that

$$
\{\Re(A+\sqrt{3} i B)\}\{\Im(i C+D)\}-\{\Im(A+\sqrt{3} i B)\}\{\Re(i C+D)\}=0 .
$$

Set $\theta=\arctan \frac{\Re(A+\sqrt{3} i B)}{\Im(A+\sqrt{3} i B)}, \lambda=\frac{2}{\sqrt{3}}(\Re A-\tan \theta \Im A), \mu=-\Re(i C)+\tan \theta \Im(i C)$ for $s$ and $\phi$ as the above. Then

$$
\Re \int_{p_{0}}^{p} e^{i \theta} G_{1}
$$

defines a conformal minimal immersion of $M$ into a flat three-torus $\mathbb{R}^{3} / \Lambda$, where $\Lambda=\cos \theta\left(\begin{array}{ccc}3 \lambda & 3 \lambda & 2 \lambda \\ \sqrt{3} \lambda & -\sqrt{3} \lambda & 0 \\ 0 & 0 & -\mu\end{array}\right)$. In particular, for $\phi=\frac{\pi}{3}$, it belongs to the $r P D$ family. 
Proof. It is sufficient to show that $L$ defined by (1) can be transformed to $\Lambda$ by $g$ and $h$ as in (2). From (A12), we have

$$
\begin{aligned}
\frac{L}{\cos \theta}=\Re\left(\begin{array}{cccccc}
2 i B & -\frac{2}{\sqrt{3}} A-2 i B & -\frac{A}{\sqrt{3}}-i B & \frac{2}{\sqrt{3}} A & \sqrt{3} A-3 i B & \frac{2}{\sqrt{3}} A-2 i B \\
-2 A & 0 & A+\sqrt{3} i B & -2 \sqrt{3} i B & A-\sqrt{3} i B & 0 \\
i C & -2 i C+D & 2 i C-D & i C-D & 0 & -D
\end{array}\right) \\
\quad-\tan \theta \Im\left(\begin{array}{cccccc}
2 i B & -\frac{2}{\sqrt{3}} A-2 i B & -\frac{A}{\sqrt{3}}-i B & \frac{2}{\sqrt{3}} A & \sqrt{3} A-3 i B & \frac{2}{\sqrt{3}} A-2 i B \\
-2 A & 0 & A+\sqrt{3} i B & -2 \sqrt{3} i B & A-\sqrt{3} i B & 0 \\
i C & -2 i C+D & 2 i C-D & i C-D & 0 & -D
\end{array}\right) .
\end{aligned}
$$

By $\tan \theta=\frac{\Re(A+\sqrt{3} i B)}{\Im(A+\sqrt{3} i B)}=\frac{\Re(i C+D)}{\Im(i C+D)}, L$ can be rewritten as

$$
L=\cos \theta\left(\begin{array}{cccccc}
-\lambda & 0 & 0 & \lambda & 3 \lambda & 2 \lambda \\
-\sqrt{3} \lambda & 0 & 0 & \sqrt{3} \lambda & \sqrt{3} \lambda & 0 \\
-\mu & 3 \mu & -3 \mu & -2 \mu & 0 & -\mu
\end{array}\right)
$$

So the theorem follows.

By using Mathematica, we find the locus of $(\phi, s)$ which satisfies (3) as in Figure 1. Set $\mathcal{D}=\left\{\left(\phi, s, x_{3}\right) \mid \phi \in\left(-\frac{\pi}{6}, \frac{\pi}{3}\right], 0.4<s<1, x_{3} \in \mathbb{R}\right\}$. The green domain is the plane $x_{3}=0$ and the blue domain is the graph of $x_{3}=\{\Re(A+\sqrt{3} i B)\}\{\Im(i C+D)\}-\{\Im(A+\sqrt{3} i B)\}\{\Re(i C+D)\}$ in $\mathcal{D}$. Hence, their intersection is precisely the locus of $(\phi, s)$ which satisfies (3) in the $(\phi, s)$-plane. Thus we obtain a numerical evidence for the existence of $(\phi, s)$ which satisfies (3), and rG family can be defined as one-parameter family of compact oriented minimal surfaces in flat three-tori, that is, $s=s(\phi)$ and $\theta=\theta(\phi)$. For $\phi=0$ and $s=\frac{1}{\sqrt{2}}$, we find $\theta=0.663483 \ldots$ and it leads to the surface known as the gyroid. For $\phi=\frac{\pi}{6}$ and $s=0.5361$, we have $\theta=0.335283 \ldots$ and it leads to the surface known as the Lidinoid. Remark that the intersection curve in Figure 1 is given in [2] (see p. 2381 in [2]). For $\phi=\frac{\pi}{3}$, we find $s=0.494722$. Numerical Result 2 in [8] implies that it corresponds to a minimal surface with nullity 4 which belongs to the rPD family.

We finally note that the green domain intersects the blue domain at the line $\phi=\frac{\pi}{3}$ in the $(\phi, s)$-plane, and it corresponds to the rPD family.
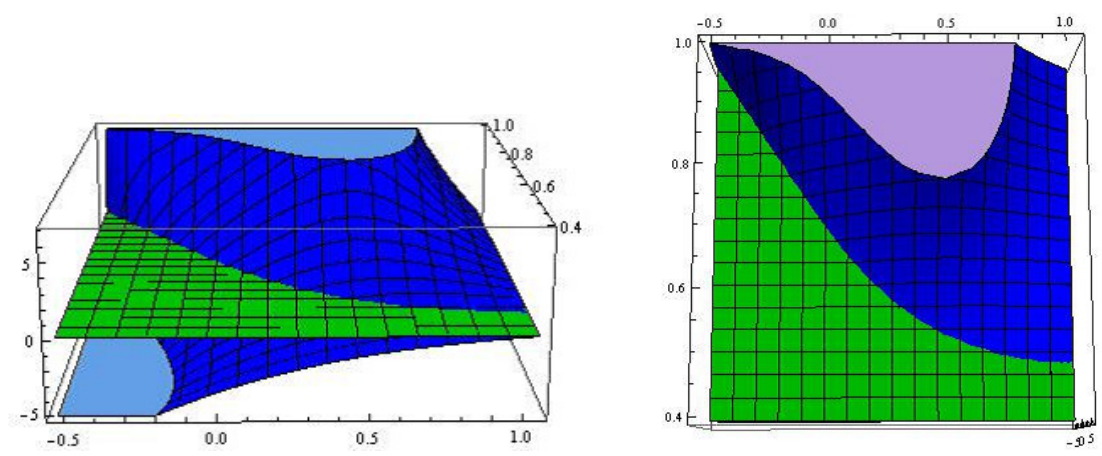

Figure 1. The locus of $(\phi, s)$ which satisfies (3) (the intersection curve of the green domain and the blue domain). 


\section{2. $t$ G Family}

For $b=s e^{i \phi}\left(0<s<1,|\phi|<\frac{\pi}{4}\right)$, let $M$ be the hyperelliptic Riemann surface of genus three defined by $w^{2}=z^{8}+a z^{4}+1=\left(z^{4}+b^{4}\right)\left(z^{4}+\frac{1}{b^{4}}\right)$. Define $G_{1}={ }^{t}\left(\frac{1-z^{2}}{w} d z, \frac{i\left(1+z^{2}\right)}{w} d z, \frac{2 z}{w} d z\right)$ as a column vector which consists of a basis of holomorphic differentials on $M$. We now use the notation as in Appendix A.2 of Appendix A. Let $\left\{A_{j}, B_{j}\right\}_{j=1}^{3}$ be the canonical homology basis on $M$ given in Appendix A.2, and set

$$
L=\Re\left\{i e^{i \theta}\left(\int_{A_{1}} G_{1} \quad \int_{A_{2}} G_{1} \quad \int_{A_{3}} G_{1} \int_{B_{1}} G_{1} \quad \int_{B_{2}} G_{1} \quad \int_{B_{3}} G_{1}\right)\right\} .
$$

We assume $s=\frac{\sqrt{3}-1}{\sqrt{2}}$ and $\theta=\arctan \frac{B}{A} \approx 0.663483 \ldots$ for $\phi=0$. In this case,

$$
\Re \int_{p_{0}}^{p} i e^{i \theta} G_{1}
$$

defines a conformal minimal immersion of $M$ into a flat three-torus $\mathbb{R}^{3} / \Lambda$ known as the gyroid, and $\Lambda$ is given by $\Lambda=\frac{A B}{\sqrt{A^{2}+B^{2}}}\left(\begin{array}{ccc}1 & 1 & 0 \\ 1 & -1 & 0 \\ 1 & 1 & 2\end{array}\right)$. In fact, we first observe that

$$
\begin{aligned}
& A=4 \int_{0}^{\infty} \frac{d x}{\sqrt{16 x^{4}-16 x^{2}+2+a}} \quad\left(x=\frac{1}{2}\left(t+\frac{1}{t}\right)\right), \\
& B=4 \int_{0}^{\infty} \frac{d x}{\sqrt{16 x^{4}+16 x^{2}+2+a}} \quad\left(x=\frac{1}{2}\left(-t+\frac{1}{t}\right)\right), \\
& C=4 \int_{0}^{\infty} \frac{d x}{\sqrt{(a+2) x^{4}+(-2 a+12) x^{2}+2+a}} \quad\left(x=\frac{1-t^{2}}{1+t^{2}}\right) .
\end{aligned}
$$

Hence, if we substitute $s=\frac{\sqrt{3}-1}{\sqrt{2}}$ and $\phi=0$, that is, $a=14$, then we find $A=C$ and $B=D$. By (A32) from Appendix A and $\tan \theta=\frac{B}{A}$, we have

$$
L=B \cos \theta\left(\begin{array}{cccccc}
1 & 1 & -1 & 1 & 2 & 1 \\
-1 & -1 & 1 & -1 & 0 & 1 \\
1 & -1 & 1 & -1 & 0 & -1
\end{array}\right)
$$

for $\phi=0$. Thus, setting

$$
g=\left(\begin{array}{cccccc}
0 & 0 & 0 & 0 & 1 & 1 \\
1 & 1 & -1 & 1 & 1 & 0 \\
0 & -1 & 1 & -1 & -1 & -1
\end{array}\right), \quad h=\left(\begin{array}{ccc}
0 & 1 & 1 \\
-1 & 0 & -1 \\
0 & 0 & 0 \\
0 & 0 & 0 \\
1 & 0 & 0 \\
0 & 0 & 0
\end{array}\right)
$$

we have $L h=\Lambda, \Lambda g=L$, and $g h=E_{3}$. We now apply Proposition 1 to $L$ given by (4) for $\phi \in\left(-\frac{\pi}{4}, \frac{\pi}{4}\right)$.

Set $L=\left(L_{1}, L_{2}, L_{3}, L_{4}, L_{5}, L_{6}\right)$, where $L_{j}$ is a $3 \times 1$ real matrix. Then, by (A32), we obtain

$$
\begin{aligned}
\text { Lhg }=L & \Longleftrightarrow L_{1}=L_{5}-L_{6}, L_{2}=-L_{3}=L_{4} \\
& \Longleftrightarrow \Re\left\{e^{i \theta}(i A+B)\right\}=\Re\left\{e^{i \theta}(i C+D)\right\}=0 \\
& \Longleftrightarrow \tan \theta=\frac{-\Im A+\Re B}{\Re A+\Im B}=\frac{-\Im C+\Re D}{\Re C+\Im D} .
\end{aligned}
$$


Therefore, we find the following result.

Theorem 3. Let $|\phi|<\frac{\pi}{4}$. Suppose that there exist s and $\phi$ such that

$$
(-\Im A+\Re B)(\Re C+\Im D)-(\Re A+\Im B)(-\Im C+\Re D)=0 .
$$

Set $\theta=\arctan \frac{-\Im A+\Re B}{\Re A+\Im B}, \lambda=\Im A+\tan \theta \Re A, \mu=\Im C+\tan \theta \Re C$ for $s$ and $\phi$ as the above. Then

$$
\Re \int_{p_{0}}^{p} i e^{i \theta} G_{1}
$$

defines a conformal minimal immersion of $M$ into a flat three-torus $\mathbb{R}^{3} / \Lambda$, where $\Lambda=\cos \theta\left(\begin{array}{ccc}\lambda & \lambda & 0 \\ \lambda & -\lambda & 0 \\ \mu & \mu & 2 \mu\end{array}\right)$. In particular, for $\phi=0$, it must be the gyroid.

Proof. It is sufficient to show that $L$ defined by (4) can be transformed to $\Lambda$ by $g$ and $h$ as in (5). From (A32), we have

$$
\frac{L}{\cos \theta}=\Re\left(\begin{array}{cccccc}
B & -i A & -B & B & 2 B & B \\
i A & -B & -i A & -B & 0 & B \\
D & -D & D & i C & 0 & i C
\end{array}\right)-\tan \theta \Im\left(\begin{array}{cccccc}
B & -i A & -B & B & 2 B & B \\
i A & -B & -i A & -B & 0 & B \\
D & -D & D & i C & 0 & i C
\end{array}\right) .
$$

By $\tan \theta=\frac{-\Im A+\Re B}{\Re A+\Im B}=\frac{-\Im C+\Re D}{\Re C+\Im D}, L$ can be rewritten as

$$
L=\cos \theta\left(\begin{array}{cccccc}
\lambda & \lambda & -\lambda & \lambda & 2 \lambda & \lambda \\
-\lambda & -\lambda & \lambda & -\lambda & 0 & \lambda \\
\mu & -\mu & \mu & -\mu & 0 & -\mu
\end{array}\right)
$$

So the theorem follows.

By using Mathematica, we find the locus of $(\phi, s)$ which satisfies (6) as in Figure 2. For $\mathcal{D}=\left\{\left(\phi, s, x_{3}\right)|| \phi \mid<\frac{\pi}{4}, 0.4<s<1, x_{3} \in \mathbb{R}\right\}$, the green domain is the plane $x_{3}=0$ and the blue domain is the graph of $x_{3}=(-\Im A+\Re B)(\Re C+\Im D)-(\Re A+\Im B)(-\Im C+\Re D)$ in $\mathcal{D}$. Hence the intersection of them is precisely the locus of $(\phi, s)$ which satisfies $(6)$ in the $(\phi, s)$-plane. Thus we obtain a numerical evidence for the existence of $(\phi, s)$ which satisfies (6), and $t G$ family can be defined as one-parameter family of compact oriented minimal surfaces in flat three-tori, that is, $s=s(\phi)$ and $\theta=\theta(\phi)$. For $\phi=0$ and $s=\frac{\sqrt{3}-1}{\sqrt{2}}$, we find $\theta=0.663483 \ldots$ and it must be the gyroid. For $\phi \rightarrow-\frac{\pi}{4}$ and $s \rightarrow 0.431882\left(s^{4}+\frac{1}{s^{4}} \approx 28.7783\right)$, we have $\theta \rightarrow \frac{\pi}{2}$ and it belongs to the tD family. Numerical Result 3 in [8] implies that it corresponds to a minimal surface with nullity 4 which belongs to the tD family. Remark that Figure 2 is given in [2] (see p. 2381 in [2]).
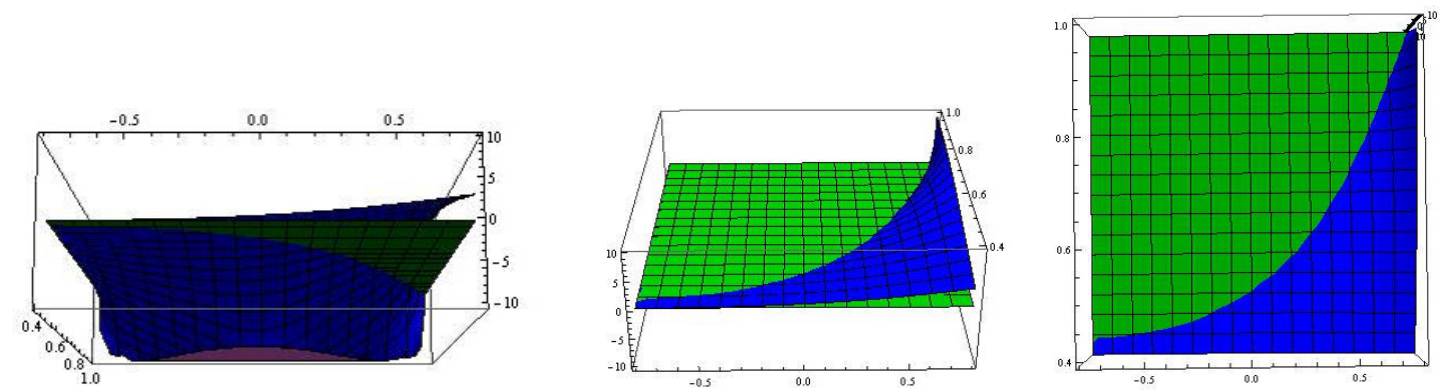

Figure 2. The locus of $(\phi, s)$ which satisfies (6) (the intersection curve of the green domain and the blue domain). 


\subsection{Embeddedness}

The embeddedness of the rG family and the $\mathrm{tG}$ family is an immediate consequence of the following theorem, which has essentially been proven by Meeks (see the proof of Theorem 7.1 in [10]).

Theorem 4. If a one-parameter family of compact oriented minimal surfaces of genus three in flat three-tori contains an embedded minimal surface in a flat three-torus, then every element of the one-parameter family must be an embedded minimal surface in a flat three-torus.

Recall that the rPD family consists of only embedded minimal surfaces in flat three-tori because it satisfies the assumption of Theorem 7.1 in [10]. The rG family contains an element of the rPD family (the case $\phi=\frac{\pi}{3}$ ), and also, the rG family meets the tG family at the gyroid. Therefore, we conclude

Theorem 5. The $r G$ family and the $t G$ family are one-parameter families of compact oriented embedded minimal surfaces of genus three in flat three-tori.

This gives an alternative proof of the following result which is obtained in [11]: the gyroid and the Lidinoid are embedded minimal surfaces in flat three-tori. In fact, the rG family contains the gyroid (the case $\phi=0$ ) and the Lidinoid (the case $\phi=\frac{\pi}{6}$ ).

\section{The Morse Indices of rG Family and tG Family}

In this section, we shall consider the Morse indices of the $\mathrm{rG}$ family and the $\mathrm{tG}$ family. The Morse index can be translated into the number of negative eigenvalues of a $18 \times 18$ real symmetric matrix counted with multiplicities and the nullity can be translated into the number of zero eigenvalues of a $9 \times 9$ Hermitian matrix counted with multiplicities (see Theorems 6 and 8). We will describe explicitly the two key matrices for the $\mathrm{rG}$ family and the $\mathrm{t} G$ family by periods of the Abelian differentials of the second kind. After that, we can compute the Morse index of the $\mathrm{rG}$ family and the tG family by numerical arguments. Let index $x_{a}$ denote the Morse index of a minimal surface.

\section{1. $r$ G Family}

We can apply the same arguments as the rPD family to the rG family since a type of a Riemann surface of the rG family coincides with that of the rPD family (see $\S 3.2$ in [8]).

We shall use the notation as in Appendix A.1 of Appendix A. Set

$$
\begin{aligned}
& P_{1}=\left(\begin{array}{ccc}
1 & 0 & -1 \\
i & 0 & i \\
0 & 2 & 0
\end{array}\right), P_{2}=\left(\begin{array}{cccccc}
\frac{1}{2} & -\frac{i}{2} & 0 & 0 & 0 & 0 \\
0 & 0 & \frac{1}{2} & 0 & 0 & 0 \\
-\frac{1}{2} & -\frac{i}{2} & 0 & 0 & 0 & 0 \\
0 & 0 & 0 & \frac{1}{2} & -\frac{i}{2} & 0 \\
0 & 0 & 0 & 0 & 0 & 1 \\
0 & 0 & 0 & -\frac{1}{2} & -\frac{i}{2} & 0
\end{array}\right), \\
& P_{a_{i}}=\left(\begin{array}{cccccc}
-\frac{5}{6 a_{i}} & -\frac{1}{2 a_{i}^{2}} & -\frac{1}{6 a_{i}^{3}} & \frac{1}{2}\left(a_{i}^{2}+\frac{1}{a_{i}^{4}}\right) & \frac{1}{2}\left(a_{i}+\frac{1}{a_{i}^{5}}\right) & \frac{1}{2}\left(1+\frac{1}{a_{i}^{6}}\right) \\
\frac{1}{6} & -\frac{1}{2 a_{i}} & -\frac{1}{6 a_{i}^{2}} & \frac{1}{2}\left(a_{i}^{3}+\frac{1}{a_{i}^{3}}\right) & \frac{1}{2}\left(a_{i}^{2}+\frac{1}{a_{i}^{4}}\right) & \frac{1}{2}\left(a_{i}+\frac{1}{a_{i}^{5}}\right) \\
\frac{a_{i}}{6} & \frac{1}{2} & -\frac{1}{6 a_{i}} & \frac{1}{2}\left(a_{i}^{4}+\frac{1}{a_{i}^{2}}\right) & \frac{1}{2}\left(a_{i}^{3}+\frac{1}{a_{i}^{3}}\right) & \frac{1}{2}\left(a_{i}^{2}+\frac{1}{a_{i}^{4}}\right)
\end{array}\right),
\end{aligned}
$$




$$
\Omega_{\mathrm{rG}}=\left(\int_{A_{1}}\left(\begin{array}{l}
G_{1} \\
G_{2}
\end{array}\right) \quad \int_{A_{2}}\left(\begin{array}{l}
G_{1} \\
G_{2}
\end{array}\right) \quad \int_{A_{3}}\left(\begin{array}{l}
G_{1} \\
G_{2}
\end{array}\right) \quad \int_{B_{1}}\left(\begin{array}{l}
G_{1} \\
G_{2}
\end{array}\right) \quad \int_{B_{2}}\left(\begin{array}{l}
G_{1} \\
G_{2}
\end{array}\right) \int_{B_{3}}\left(\begin{array}{l}
G_{1} \\
G_{2}
\end{array}\right)\right)
$$

and choose $a_{1}=a, a_{2}=e^{\frac{2}{3} \pi i} a, a_{3}=e^{\frac{4}{3} \pi i} a, a_{4}=-1 / a, a_{5}=-e^{\frac{2}{3} \pi i} / a$. We define $\left\{T_{j}\right\}_{j=1}^{5}:=\left\{\frac{1}{2} P_{1} P_{a_{j}} P_{2} \Omega_{\mathrm{rG}}\right\}_{j=1}^{5}$.

Setting $C_{1}$ and $C_{2}$ are $3 \times 3$ complex matrices given by

$$
\left(C_{1}, C_{2}\right)=\left(\begin{array}{cccccc}
2 i B & -\frac{2}{\sqrt{3}} A-2 i B & -\frac{A}{\sqrt{3}}-i B & \frac{2}{\sqrt{3}} A & \sqrt{3} A-3 i B & \frac{2}{\sqrt{3}} A-2 i B \\
-2 A & 0 & A+\sqrt{3} i B & -2 \sqrt{3} i B & A-\sqrt{3} i B & 0 \\
i C & -2 i C+D & 2 i C-D & i C-D & 0 & -D
\end{array}\right)
$$

we have the Riemann matrix $\tau=C_{1}^{-1} C_{2}$ and define

$$
\begin{aligned}
& T_{6}:=\left(C_{1}, C_{2}\right), \quad T_{7}:=\left(\begin{array}{ccc}
0 & 1 & 0 \\
-1 & 0 & 0 \\
0 & 0 & 0
\end{array}\right)\left(C_{1}, C_{2}\right) \\
& T_{8}:=\left(\begin{array}{ccc}
0 & 0 & 1 \\
0 & 0 & 0 \\
-1 & 0 & 0
\end{array}\right)\left(C_{1}, C_{2}\right), \quad T_{9}:=\left(\begin{array}{ccc}
0 & 0 & 0 \\
0 & 0 & 1 \\
0 & -1 & 0
\end{array}\right)\left(C_{1}, C_{2}\right) .
\end{aligned}
$$

Decompose $T_{j}=\left(Z_{j 1}, Z_{j 2}\right)$ into two $3 \times 3$ complex matrices. We introduce

$$
\eta\left(\left(Z_{j 1}, Z_{j 2}\right),\left(Z_{j 1}, Z_{j 2}\right)\right)=-i \operatorname{tr}\left({ }^{t} Z_{j 2} \overline{Z_{j 1}}-{ }^{t} Z_{j 1} \overline{Z_{j 2}}\right)
$$

and set $W=\left(\eta\left(T_{i}, T_{j}\right)\right) . W$ is a $9 \times 9$ Hermitian matrix and one of the two key matrices. Let $(p, q)$ denote the signature of $W$.

For a decomposition $T_{j}=\left(Z_{j 1}, Z_{j 2}\right)$, we set

$$
K_{j}^{\prime}=\Re Z_{j 1}+i\left\{\Re Z_{j 1} \Re \tau-\Re Z_{j 2}\right\}(\Im \tau)^{-1}, \quad K_{j}^{\prime \prime}=\Re\left(i Z_{j 1}\right)+i\left\{\Re\left(i Z_{j 1}\right) \Re \tau-\Re\left(i Z_{j 2}\right)\right\}(\Im \tau)^{-1} .
$$

Define

$$
U_{j}=\left\{\begin{array}{ll}
T_{j} & (1 \leq j \leq 9) \\
i T_{j-9} & (10 \leq j \leq 18)
\end{array} \quad \quad V_{j}= \begin{cases}\left(K_{j}^{\prime}, K_{j}^{\prime} \tau\right) & (1 \leq j \leq 9) \\
\left(K_{j-9}^{\prime \prime}, K_{j-9}^{\prime \prime} \tau\right) & (10 \leq j \leq 18)\end{cases}\right.
$$

and $W_{1}=\left(\Re\left(\eta\left(U_{i}, U_{j}\right)\right)\right), W_{2}=\left(\Re\left(\eta\left(V_{i}, V_{j}\right)\right)\right) . W_{2}-W_{1}$ is a $18 \times 18$ real symmetric matrix and the another key matrix.

The next theorem follows from Theorem 7.10 and $\S 14$ in [5], Theorems 5.4, 5.5, and 6.3 in [6] (see also $\S 2.6$ in [7]).

Theorem 6. The nullity of the $r G$ family is equal to the number of zero eigenvalues of $W$, counted with multiplicities plus 3. Moreover, if the number of zero eigenvalues of $W_{2}-W_{1}$ is equal to 8 , then the Morse index of the $r G$ family is equal to the number of negative eigenvalues of $W_{2}-W_{1}$, counted with multiplicities plus 1.

For $\mathcal{D}=\left\{\left(\phi, s, x_{3}\right) \mid \phi \in\left(-\frac{\pi}{6}, \frac{\pi}{3}\right], 0.4<s<1, x_{3} \in \mathbb{R}\right\}$, by using Mathematica, we obtain the graph of $x_{3}=-\operatorname{det}(W)$ and the plane $x_{3}=0$ in $\mathbb{R}^{3}$ (see Figures 3 and 4 ). The intersection of them consists of the points whose nullities are at least 4 . 
The intersection of the three graphs of $x_{3}=-\operatorname{det}(W)$, the plane $x_{3}=0$, and $x_{3}=\{\Re(A+$ $\sqrt{3} i B)\}\{\Im(i C+D)\}-\{\Im(A+\sqrt{3} i B)\}\{\Re(i C+D)\}$ in $\mathcal{D}$ is given in Figure 5.

From Figure 5, we can show that the locus of $(\phi, s)$ which satisfies (3) contains two connected components (see Figure 6). In fact, we will numerically compute the eigenvalues of $W$ and $W_{2}-W_{1}$.

We first consider the eigenvalues of $W$. Substituting $(\phi, s)=\left(-\frac{\pi}{6.01}, 0.45\right),\left(-\frac{\pi}{6.01}, 0.6\right)$, $\left(-\frac{\pi}{6.01}, 0.9\right)$ to $W$ yields the following sets of the eigenvalues:

$$
\begin{aligned}
& \{152.006,83.3364,71.4652,61.5538,-4.94045,-3.35369,0.49079,-0.144368,-0.0369606\}, \\
& \{188.14,111.028,110.934,94.906,-4.81546,-4.40164,-1.81147,-0.204606,-0.0535919\}, \\
& \{369.902,254.671,215.325,179.236,-51.9581,-38.125,-24.2347,1.33455,0.354338\} .
\end{aligned}
$$
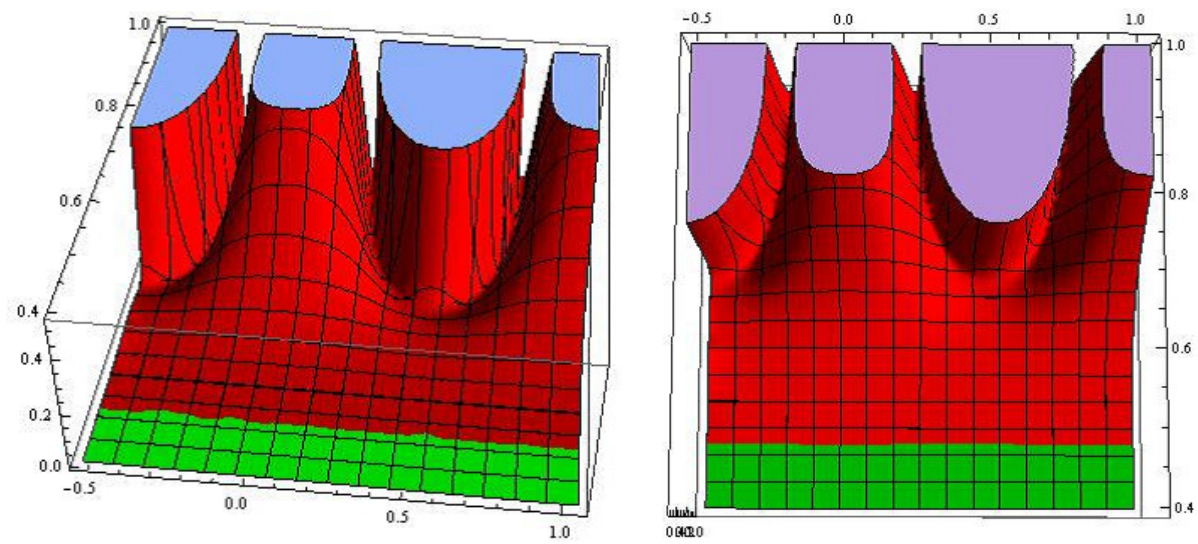

Figure 3. The graph of $x_{3}=-\operatorname{det} W$ (the red domain) and the plane $x_{3}=0$ (the green domain) in $\mathcal{D}$. They intersect at a curve which looks like a straight line.
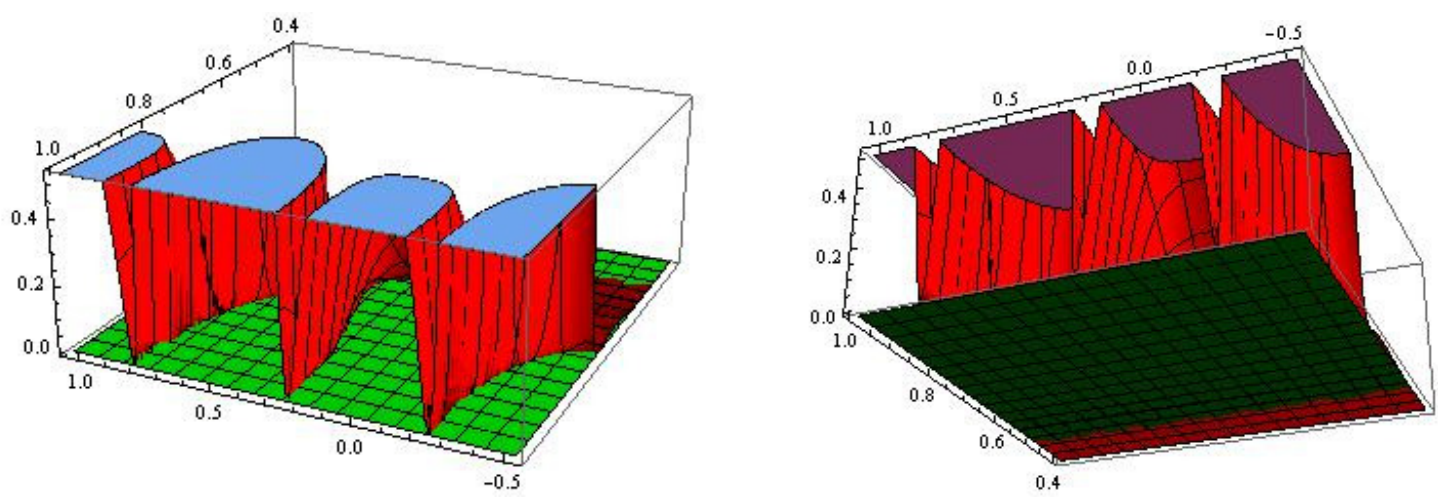

Figure 4. The graph of $x_{3}=-\operatorname{det} W$ (the red domain) and the plane $x_{3}=0$ (the green domain) in $\mathcal{D}$. The former seems to be tangent to the latter at some curves which look like parabolic curves. 

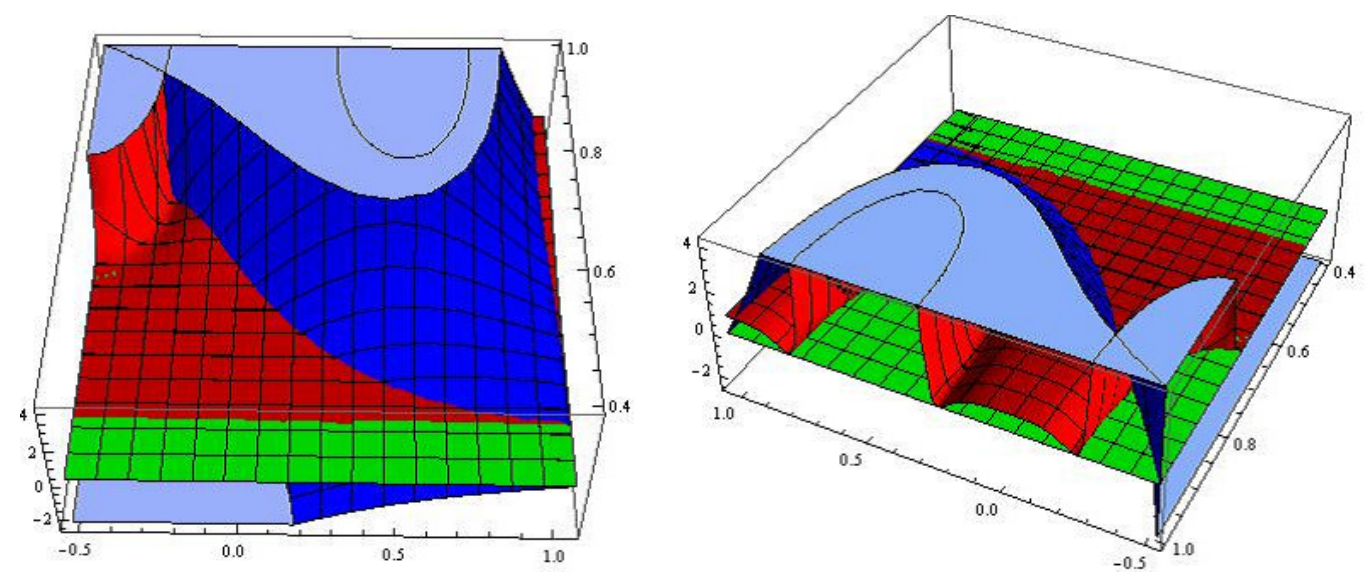

Figure 5. The graph of $x_{3}=-\operatorname{det} W$ (the red domain), the plane $x_{3}=0$ (the green domain), and $x_{3}=$ $\{\Re(A+\sqrt{3} i B)\}\{\Im(i C+D)\}-\{\Im(A+\sqrt{3} i B)\}\{\Re(i C+D)\}$ (the blue domain) in $\mathcal{D}$.

Next, we substitute $(\phi, s)=(0,0.45),(0,0.9)$ to $W$ and obtain the following sets of the eigenvalues:

$$
\begin{aligned}
& \{152.402,82.803,70.8832,61.6518,-4.7351,-3.21415,0.459665,-0.163676,-0.0419736\}, \\
& \{265.411,151.782,145.946,134.336,-7.37701,-3.5439,-2.57836,-0.805086,-0.181996\} .
\end{aligned}
$$

Moreover, substituting $(\phi, s)=\left(\frac{\pi}{6}, 0.45\right),\left(\frac{\pi}{6}, 0.6\right),\left(\frac{\pi}{6}, 0.9\right)$ to $W$, we have the following sets of the eigenvalues:

$\{145.716,73.5343,59.8217,51.8676,-5.45245,-3.07576,0.836401,-0.106013,-0.0253394\}$,

$\{188.14,111.028,110.934,94.906,-4.81547,-4.40165,-1.81147,-0.204605,-0.0535916\}$,

$\{369.906,254.676,215.327,179.237,-51.961,-38.1271,-24.236,1.33459,0.354349\}$.

Finally, we substitute $(\phi, s)=\left(\frac{\pi}{3}, 0.45\right),\left(\frac{\pi}{3}, 0.9\right)$ to $W$ and find the following sets of the eigenvalues:

$\{145.971,73.3025,59.586,51.9706,-5.34193,-3.0093,0.815662,-0.113851,-0.0272483\}$,

$\{265.411,151.782,145.946,134.336,-7.37701,-3.5439,-2.57836,-0.805086,-0.181996\}$.

We now consider the eigenvalues of $W_{2}-W_{1}$. Substituting $(\phi, s)=\left(-\frac{\pi}{6.01}, 0.45\right),\left(-\frac{\pi}{6.01}, 0.6\right)$, $\left(-\frac{\pi}{6.01}, 0.9\right)$ to $W_{2}-W_{1}$ yields the following sets of the eigenvalues:

$\{14.5758,14.549,10.0379,9.97935,8.89303,-0.709172,0.543324,0.245049,0.128432$, $0.0581318,0,0,0,0,0,0,0,0\}$,

$\{16.6566,16.5459,14.8343,10.5963,10.293,2.65305,1.62215,0.520714,0.2287$, $0.0775775,0,0,0,0,0,0,0,0\}$,

$\{146.521,129.728,114.312,106.675,70.2955,70.1246,4.00972,1.3252,-1.06449$, $-0.357892,0,0,0,0,0,0,0,0\}$. 
Next, we substitute $(\phi, s)=(0,0.45),(0,0.9)$ to $W_{2}-W_{1}$ and obtain the following sets of the eigenvalues:

$\{14.1866,14.1478,9.71217,9.70707,8.64341,-0.670382,0.496307,0.269501,0.131996$, $0.0715017,0,0,0,0,0,0,0,0\}$,

$\{18.0216,14.5156,14.1481,13.3706,10.1915,8.73066,1.82703,1.44482,0.580926$, $0.434436,0,0,0,0,0,0,0,0\}$.

Moreover, substituting $(\phi, s)=\left(\frac{\pi}{6}, 0.45\right),\left(\frac{\pi}{6}, 0.6\right),\left(\frac{\pi}{6}, 0.9\right)$ to $W_{2}-W_{1}$, we have the following sets of eigenvalues:

$\{14.5758,14.549,10.0379,9.97935,8.89303,-0.709172,0.543324,0.245049,0.128432$, $0.0581317,0,0,0,0,0,0,0,0\}$,

$\{16.6566,16.5459,14.8343,10.5963,10.293,2.65306,1.62215,0.520714,0.228698$, $0.077577,0,0,0,0,0,0,0,0\}$,

$\{146.529,129.736,114.319,106.682,70.2996,70.1288,4.00972,1.3252,-1.06451$, $-0.3579,0,0,0,0,0,0,0,0\}$.

Finally, we substitute $(\phi, s)=\left(\frac{\pi}{3}, 0.45\right),\left(\frac{\pi}{3}, 0.9\right)$ to $W_{2}-W_{1}$ and find the following sets of the eigenvalues:

$\{14.1866,14.1478,9.71217,9.70707,8.64341,-0.670382,0.496307,0.269501,0.131996$, $0.0715017,0,0,0,0,0,0,0,0\}$,

$\{18.0216,14.5156,14.1481,13.3706,10.1915,8.73066,1.82703,1.44482,0.580926$,

$0.434436,0,0,0,0,0,0,0,0\}$.

Therefore, we conclude that (i) $(p, q)=(6,3)$ and index $x_{a}=3$ on the domain $A$, (ii) $(p, q)=(4,5)$ and index $x_{a}=1$ on the domain $B$, (iii) $(p, q)=(5,4)$ and index $_{a}=2$ on the domain $C$ in Figure 6 . Hence, we obtain the following result.

Theorem 7. The $r G$ family contains minimal surfaces with $(p, q)=(6,3)$, index $x_{a}=3$ and minimal surfaces with $(p, q)=(4,5)$, index $x_{a}=1$.
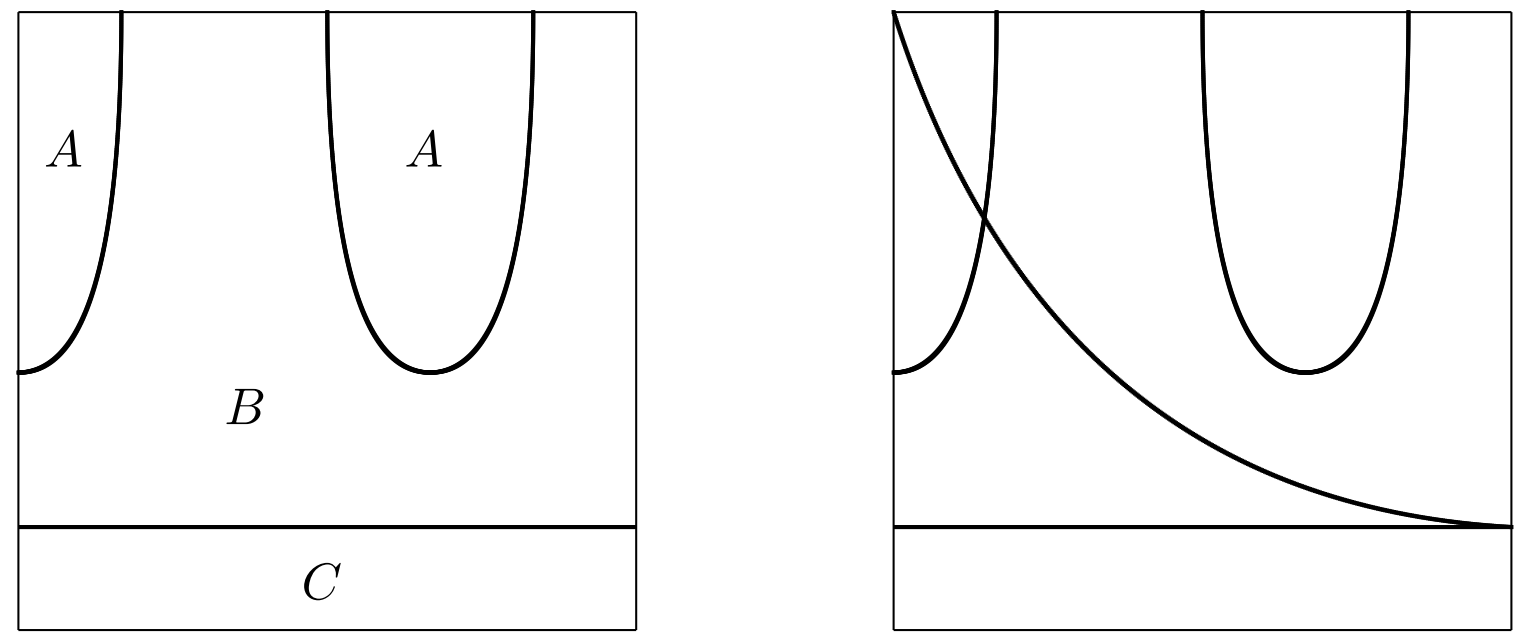

Figure 6. The locus $\operatorname{det}(W)=0$ and that of $(\phi, s)$ which satisfies (3) in $\left\{(\phi, s) \mid \phi \in\left(-\frac{\pi}{6}, \frac{\pi}{3}\right], 0.4<s<1\right\}$. 
Remark 1. It is clear to see that the graph of $x_{3}=\operatorname{det}(W)$ intersects the plane $x_{3}=0$ at a curve which looks like a straight line as in Figure 3 by Mathematica. The intersection curve is the locus at which the jump of the Morse index is equal to 1 . On the other hand, the situation of Figure 4 is quite different. We obtain numerical evidence of the existence of two kinds of minimal surfaces, namely, the minimal surface with Morse index 1 and the minimal surface with Morse index 3. So there must exist a boundary at which the jump of the Morse index is equal to 2, and it remains an important problem to study such boundaries, which are more complicated than the above. Hence there might be error terms of the numerical approximation, and it is not clear to check the graph of $x_{3}=\operatorname{det}(W)$ is tangent to the plane $x_{3}=0$ at some curves which look like parabolic curves as in Figure 4 by Mathematica.

\section{2. $t$ G Family}

We can apply the same arguments as the tP family to the $t G$ family since a type of Riemann surface of the $t G$ family coincides with that of the $t P$ family (see $\S 3.3$ in [8]).

We shall use the notation as in Appendix A.2 of Appendix A. Set

$$
\begin{aligned}
& P_{1}=\left(\begin{array}{ccc}
1 & 0 & -1 \\
i & 0 & i \\
0 & 2 & 0
\end{array}\right), \quad P_{2}=\left(\begin{array}{cccccc}
\frac{1}{2} & -\frac{i}{2} & 0 & 0 & 0 & 0 \\
0 & 0 & \frac{1}{2} & 0 & 0 & 0 \\
-\frac{1}{2} & -\frac{i}{2} & 0 & 0 & 0 & 0 \\
0 & 0 & 0 & \frac{1}{2} & -\frac{i}{2} & 0 \\
0 & 0 & 0 & 0 & 0 & 1 \\
0 & 0 & 0 & -\frac{1}{2} & -\frac{i}{2} & 0
\end{array}\right), \\
& P_{a_{i}}=\left(\begin{array}{cccccc}
-\frac{3}{4 a_{i}} & -\frac{1}{2 a_{i}^{2}} & -\frac{1}{4 a_{i}^{3}} & \frac{a}{2 a_{i}}+a_{i}^{3} & \frac{a}{2 a_{i}^{2}}+a_{i}^{2} & \frac{a}{2 a_{i}^{3}}+a_{i} \\
\frac{1}{4} & -\frac{1}{2 a_{i}} & -\frac{1}{4 a_{i}^{2}} & -\frac{a}{2}-\frac{1}{a_{i}^{4}} & \frac{a}{2 a_{i}}+a_{i}^{3} & \frac{a}{2 a_{i}^{2}}+a_{i}^{2} \\
\frac{a_{i}}{4} & \frac{1}{2} & -\frac{1}{4 a_{i}} & -\frac{a}{2} a_{i}-\frac{1}{a_{i}^{3}} & -\frac{a}{2}-\frac{1}{a_{i}^{4}} & \frac{a}{2 a_{i}}+a_{i}^{3}
\end{array}\right), \\
& \Omega_{\mathrm{tG}}=\left(\int_{A_{1}}\left(\begin{array}{l}
G_{1} \\
G_{2}
\end{array}\right) \int_{A_{2}}\left(\begin{array}{l}
G_{1} \\
G_{2}
\end{array}\right) \int_{A_{3}}\left(\begin{array}{l}
G_{1} \\
G_{2}
\end{array}\right) \int_{B_{1}}\left(\begin{array}{l}
G_{1} \\
G_{2}
\end{array}\right) \int_{B_{2}}\left(\begin{array}{l}
G_{1} \\
G_{2}
\end{array}\right) \int_{B_{3}}\left(\begin{array}{l}
G_{1} \\
G_{2}
\end{array}\right)\right)
\end{aligned}
$$

and choose $a_{1}=e^{\frac{\pi}{4} i} b, a_{2}=e^{\frac{3}{4} \pi i} b, a_{3}=e^{-\frac{\pi}{4} i} b, a_{4}=e^{-\frac{3}{4} \pi i} b$, and $a_{5}=e^{\frac{\pi}{4} i} / b$. We define $\left\{T_{j}\right\}_{j=1}^{5}:=\left\{\frac{1}{2} P_{1} P_{a_{j}} P_{2} \Omega_{\mathrm{tG}}\right\}_{j=1}^{5}$.

Setting $C_{1}$ and $C_{2}$ are $3 \times 3$ complex matrices given by

$$
\left(C_{1}, C_{2}\right)=\left(\begin{array}{cccccc}
-i B & -A & i B & -i B & -2 i B & -i B \\
A & i B & -A & i B & 0 & -i B \\
-i D & i D & -i D & C & 0 & C
\end{array}\right)
$$

we have the Riemann matrix $\tau=C_{1}^{-1} C_{2}$ and define

$$
\begin{aligned}
& T_{6}:=\left(C_{1}, C_{2}\right), \quad T_{7}:=\left(\begin{array}{ccc}
0 & 1 & 0 \\
-1 & 0 & 0 \\
0 & 0 & 0
\end{array}\right)\left(C_{1}, C_{2}\right), \\
& T_{8}:=\left(\begin{array}{ccc}
0 & 0 & 1 \\
0 & 0 & 0 \\
-1 & 0 & 0
\end{array}\right)\left(C_{1}, C_{2}\right), \quad T_{9}:=\left(\begin{array}{ccc}
0 & 0 & 0 \\
0 & 0 & 1 \\
0 & -1 & 0
\end{array}\right)\left(C_{1}, C_{2}\right) .
\end{aligned}
$$


Decompose $T_{j}=\left(Z_{j 1}, Z_{j 2}\right)$ into two $3 \times 3$ complex matrices. We introduce

$$
\eta\left(\left(Z_{j 1}, Z_{j 2}\right),\left(Z_{j 1}, Z_{j 2}\right)\right)=-i \operatorname{tr}\left({ }^{t} Z_{j 2} \overline{Z_{j 1}}-{ }^{t} Z_{j 1} \overline{Z_{j 2}}\right)
$$

and set $W=\left(\eta\left(T_{i}, T_{j}\right)\right)$. Let $(p, q)$ denote the signature of $W$.

For a decomposition $T_{j}=\left(Z_{j 1}, Z_{j 2}\right)$, we set

$$
K_{j}^{\prime}=\Re Z_{j 1}+i\left\{\Re Z_{j 1} \Re \tau-\Re Z_{j 2}\right\}(\Im \tau)^{-1}, \quad K_{j}^{\prime \prime}=\Re\left(i Z_{j 1}\right)+i\left\{\Re\left(i Z_{j 1}\right) \Re \tau-\Re\left(i Z_{j 2}\right)\right\}(\Im \tau)^{-1} .
$$

Define

$$
U_{j}=\left\{\begin{array}{ll}
T_{j} & (1 \leq j \leq 9) \\
i T_{j-9} & (10 \leq j \leq 18)
\end{array}, \quad V_{j}= \begin{cases}\left(K_{j}^{\prime}, K_{j}^{\prime} \tau\right) & (1 \leq j \leq 9) \\
\left(K_{j-9}^{\prime \prime}, K_{j-9}^{\prime \prime} \tau\right) & (10 \leq j \leq 18)\end{cases}\right.
$$

and $W_{1}=\left(\Re\left(\eta\left(U_{i}, U_{j}\right)\right)\right), W_{2}=\left(\Re\left(\eta\left(V_{i}, V_{j}\right)\right)\right)$.

Theorem 8. The nullity of the $t G$ family is equal to the number of zero eigenvalues of $W$, counted with multiplicities plus 3. Moreover, if the number of zero eigenvalues of $W_{2}-W_{1}$ is equal to 8 , then the Morse index of the $t G$ family is equal to the number of negative eigenvalues of $W_{2}-W_{1}$, counted with multiplicities plus 1 .

For $\mathcal{D}=\left\{\left(\phi, s, x_{3}\right)|| \phi \mid<\frac{\pi}{4}, 0.4<s<1, x_{3} \in \mathbb{R}\right\}$, by using Mathematica, we obtain the graph of $x_{3}=\operatorname{det}(W)$ and the plane $x_{3}=0$ in $\mathcal{D}$ (see Figure 7). Their intersection consists of the points whose nullities are at least 4 .
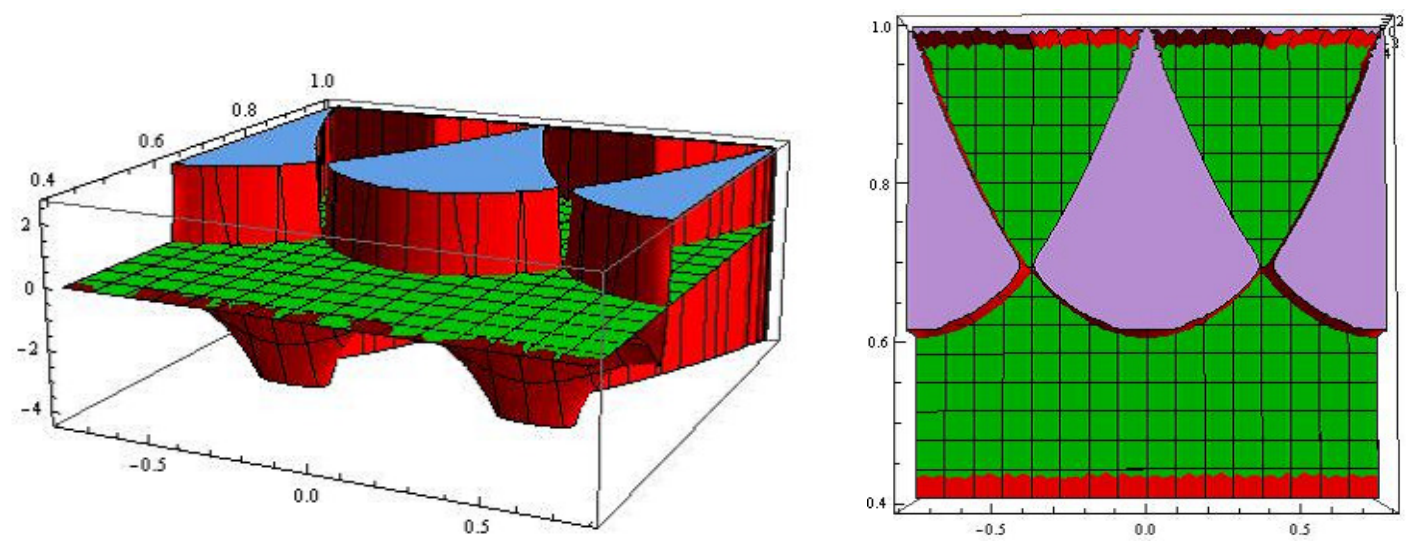

Figure 7. The graph of $x_{3}=\operatorname{det} W$ (the red domain) and the plane $x_{3}=0$ (the green domain) in $\mathcal{D}$.

They intersect at a curve which looks like a straight line and curves which look like parabolic curves.

The intersection of the three graphs of $x_{3}=\operatorname{det}(W)$, the plane $x_{3}=0$, and $x_{3}=(-\Im A+\Re B)(\Re C+\Im D)-(\Re A+\Im B)(-\Im C+\Re D)$ in $\mathcal{D}$ is given in Figure 8.

From Figure 8, we can show that the locus of $(\phi, s)$ which satisfies (6) contains two connected components. In fact, we will numerically compute the eigenvalues of $W$ and $W_{2}-W_{1}$.

We first consider the eigenvalues of $W$. Substituting $(\phi, s)=\left( \pm \frac{\pi}{4.1}, 0.4\right),\left( \pm \frac{\pi}{4.1}, 0.5\right),\left( \pm \frac{\pi}{4.1}, 0.8\right)$ to $W$ yields the following sets of the eigenvalues:

$\{35.6189,22.208,20.1174,15.8479,-2.53191,-1.47707,0.0950535,-0.042007,-0.00973469\}$,

$\{51.9452,38.3399,31.0118,27.6411,-2.29972,-2.08639,-0.350738,-0.143488,-0.0187563\}$ ，

$\{140.144,119.05,87.6919,73.3903,-9.2786,-7.68567,-3.04764,1.18144,-0.372079\}$. 
Next, we substitute $(\phi, s)=\left( \pm \frac{\pi}{8}, 0.4\right),\left( \pm \frac{\pi}{8}, 0.5\right),\left( \pm \frac{\pi}{8}, 0.8\right)$ to $W$ and obtain the following sets of the eigenvalues:

$\{35.6372,22.1863,20.103,15.8582,-2.52123,-1.47505,0.0946025,-0.0420784,-0.00980311\} ，$ $\{52.0196,38.1231,30.8936,27.6668,-2.27561,-2.0269,-0.347043,-0.143621,-0.0201313\}$ ， $\{131.882,96.7425,81.38,65.5126,-5.37746,-4.23261,-1.94126,1.42518,0.0835739\}$.
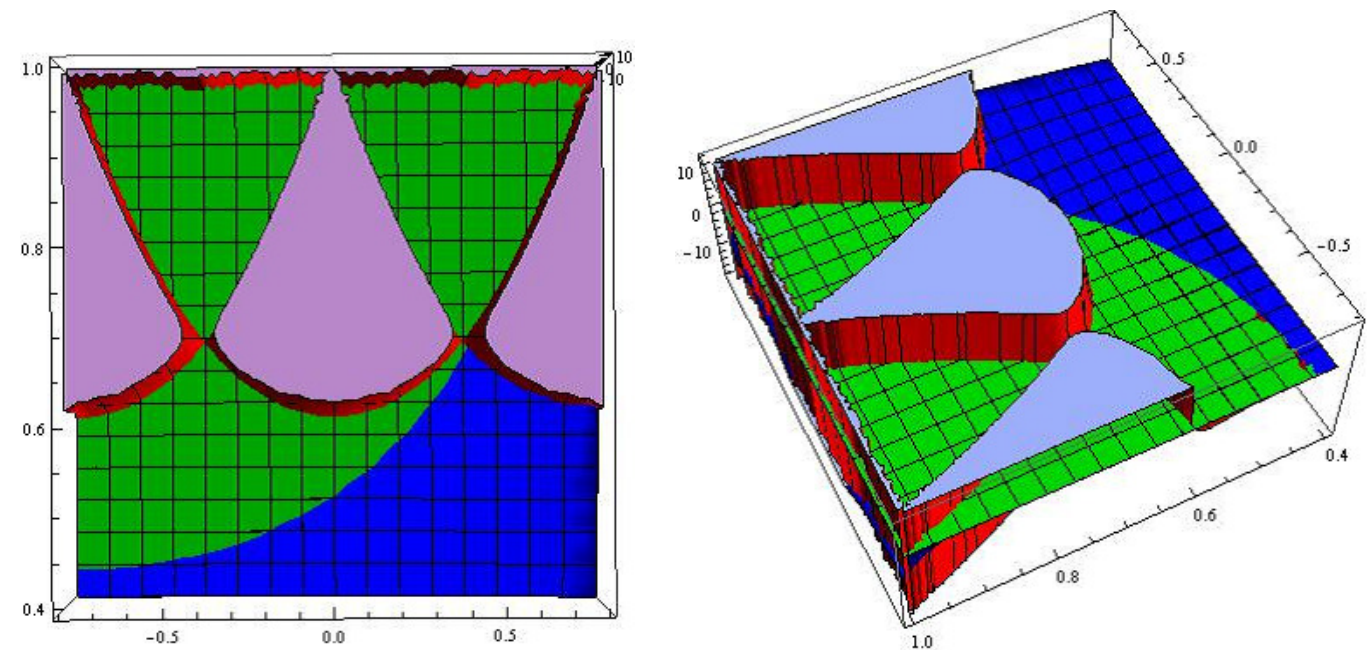

Figure 8. The graph of $x_{3}=\operatorname{det} W$ (the red domain), the plane $x_{3}=0$ (the green domain), and $x_{3}=(-\Im A+\Re B)(\Re C+\Im D)-(\Re A+\Im B)(-\Im C+\Re D)$ (the blue domain) in $\mathcal{D}$.

Moreover, substituting $(\phi, s)=(0,0.4),(0,0.5),(0,0.8)$ to $W$, we have the following sets of eigenvalues:

$\{35.6188,22.2081,20.1175,15.8479,-2.53197,-1.47708,0.0950562,-0.0420066,-0.00973429\}$,

$\{51.9447,38.3412,31.0125,27.641,-2.29986,-2.08675,-0.350761,-0.143487,-0.0187482\}$,

$\{140.187,119.276,87.7299,73.461,-9.32263,-7.72632,-3.0591,1.17902,-0.379217\}$.

We now consider the eigenvalues of $W_{2}-W_{1}$. Substituting $(\phi, s)=\left( \pm \frac{\pi}{4.1}, 0.4\right),\left( \pm \frac{\pi}{4.1}, 0.5\right)$, $\left( \pm \frac{\pi}{4.1}, 0.8\right)$ to $W_{2}-W_{1}$ yields the following sets of eigenvalues:

$\{7.77508,4.38731,4.37729,3.7917,2.34541,-0.145468,0.112206,0.102314,0.0342688$, $0.0177381,0,0,0,0,0,0,0,0\}$,

$\{8.96534,6.10147,6.05441,4.04023,2.79856,0.544306,0.394305,0.367723,0.0998041$, $0.0287088,0,0,0,0,0,0,0,0\}$,

$\{24.074,21.1387,19.5594,19.2666,14.565,8.19564,7.48133,0.960304,-0.71742$, $0.568975,0,0,0,0,0,0,0,0\}$. 
Next, we substitute $(\phi, s)=\left( \pm \frac{\pi}{8}, 0.4\right),\left( \pm \frac{\pi}{8}, 0.5\right),\left( \pm \frac{\pi}{8}, 0.8\right)$ to $W_{2}-W_{1}$ and obtain the following sets of the eigenvalues:

$\{7.75667,4.37796,4.368,3.78497,2.34032,-0.144906,0.111943,0.102423,0.0339594$ ， $0.0180341,0,0,0,0,0,0,0,0\}$,

$\{8.83532,6.02283,5.97696,3.98793,2.77818,0.539496,0.389623,0.366682,0.0950817$, $0.032076,0,0,0,0,0,0,0,0\}$,

$\{13.5834,10.9767,10.9737,10.1408,8.8041,5.17527,4.61406,2.22136,-0.489929$, $-0.115717,0,0,0,0,0,0,0,0\}$.

Moreover, substituting $(\phi, s)=(0,0.4),(0,0.5),(0,0.8)$ to $W_{2}-W_{1}$, we have the following sets of the eigenvalues:

$\{7.77519,4.38737,4.37734,3.79174,2.34544,-0.145471,0.112207,0.102313,0.0342706$, $0.0177364,0,0,0,0,0,0,0,0\}$,

$\{8.96612,6.10194,6.05487,4.04054,2.79869,0.544335,0.394332,0.367729,0.0998308$, $0.0286902,0,0,0,0,0,0,0,0\}$,

$\{24.1893,21.2568,19.6545,19.3614,14.6312,8.22985,7.5127,0.924861,-0.71973$, $0.598225,0,0,0,0,0,0,0,0\}$.

Therefore, we conclude that (i) $(p, q)=(6,3)$ and index $x_{a}=3$ on the domain $A$, (ii) $(p, q)=(5,4)$ and index $_{a}=2$ on the domain $B$, (iii) $(p, q)=(4,5)$ and index $_{a}=1$ on the domain $C$ in Figure 9. Hence, we obtain the following result.

Theorem 9. The $t G$ family contains minimal surfaces with $(p, q)=(5,4)$, index $x_{a}=2$ and minimal surfaces with $(p, q)=(4,5)$, index $x_{a}=1$.
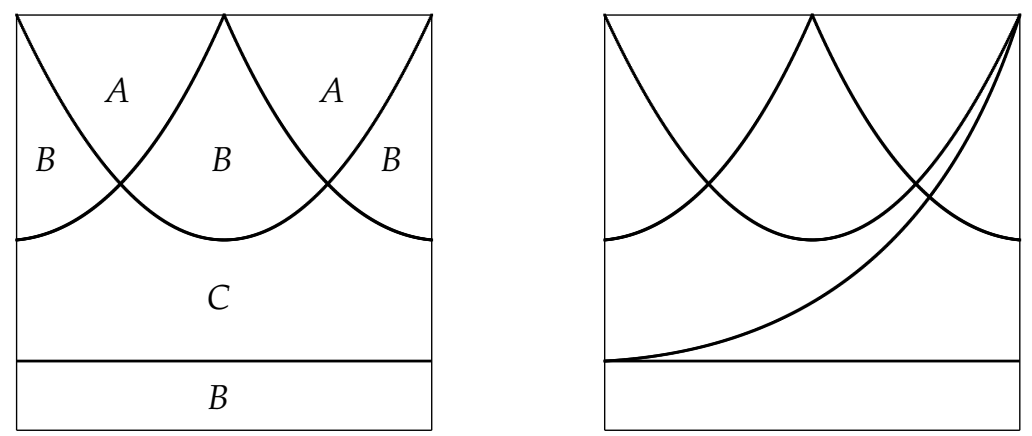

Figure 9. The locus $\operatorname{det}(W)=0$ and that of $(\phi, s)$ which satisfies (6) in $\left\{(\phi, s)|| \phi \mid<\frac{\pi}{4}, 0.4<s<1\right\}$.

\subsection{A One-Parameter Family Which Contains P Surface, D Surface, Gyroid, Lidinoid}

Schröder-Turk, Fogden, and Hyde [12] gave Figure 10 as a correlation diagram for the seven one-parameter families, namely, the $\mathrm{H}$ family, the rPD family, the tP family, the tD family, the tCLP family, the $\mathrm{rG}$ family, the $t G$ family. Every line implies a one-parameter family, for example, the line labeled $\mathrm{H}$ indicates the $\mathrm{H}$ family, and so on.

From Numerical Result 1, Numerical Result 2, Numerical Result 3 in [8], we can see the minimal surfaces with $i n d e x_{a}=1$ for the $\mathrm{H}$ family, the rPD family, the $\mathrm{tP}$ family, the $\mathrm{tD}$ family. Combining these results, Theorems 7 and 9 yield the red lines in Figure 10. Therefore we have 
Theorem 10. The P surface, the D surface, the gyroid, the Lidinoid are contained in a one-parameter family which consists of minimal surfaces with index $x_{a}=1$.

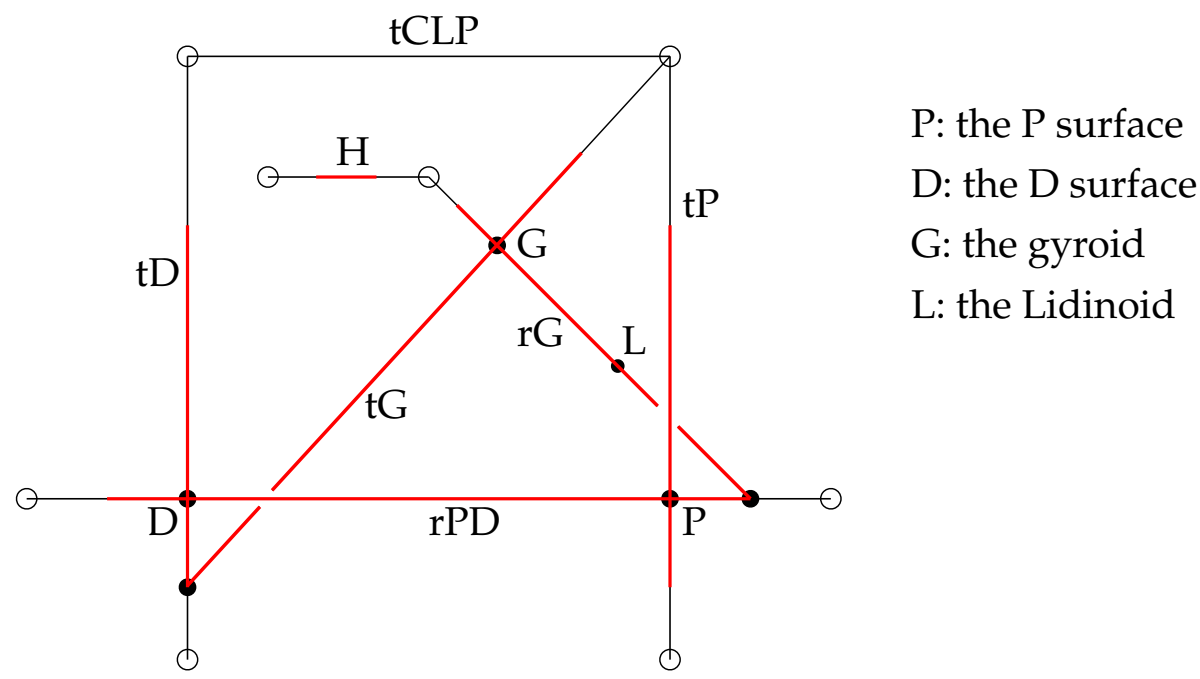

Figure 10. The diagram of the $\mathrm{H}$ family, the rPD family, the tP family, the tD family, the tCLP family, the $\mathrm{rG}$ family, the $\mathrm{tG}$ family (the red lines indicate minimal surfaces with index $x_{a}=1$ ).

Author Contributions: Conceptualization, N.E. and T.S.; methodology, N.E. and T.S.; software, N.E. and T.S.; validation, N.E. and T.S.; formal analysis, N.E. and T.S.; investigation, N.E. and T.S.; data curation, N.E. and T.S.; writing - original draft preparation, N.E. and T.S.; writing — review and editing, N.E. and T.S.; visualization, N.E. and T.S.; project administration, N.E. and T.S. All authors have read and agreed to the published version of the manuscript.

Funding: This research received no external funding.

Conflicts of Interest: The authors declare no conflict of interest.

\section{Appendix A. List of Period Calculations}

We shall determine canonical homology bases and present period calculations of the Abelian differentials of the second kind along the canonical homology bases for the $\mathrm{rG}$ family and the $\mathrm{t} G$ family as an appendix.

\section{Appendix A.1. rG Family}

For $a=s e^{i \phi}\left(0<s<1, \phi \in\left(-\frac{\pi}{6}, \frac{\pi}{3}\right]\right)$, let $M$ be a hyperelliptic Riemann surface of genus three defined by $w^{2}=z\left(z^{3}-a^{3}\right)\left(z^{3}+\frac{1}{a^{3}}\right)$.

Define $G_{1}:={ }^{t}\left(\frac{1-z^{2}}{w} d z, \frac{i\left(1+z^{2}\right)}{w} d z, \frac{2 z}{w} d z\right)$ as a column vector which consists of a basis of holomorphic differentials on $M$. Up to exact one-forms, the Abelian differentials of the second kind are given by

$$
\left\{\frac{1-z^{2}}{w} d z, \frac{i\left(1+z^{2}\right)}{w} d z, \frac{2 z}{w} d z, \frac{z^{4}-z^{6}}{w^{3}} d z, \frac{i\left(z^{4}+z^{6}\right)}{w^{3}} d z, \frac{z^{5}}{w^{3}} d z\right\}
$$

and we set $G_{2}={ }^{t}\left(\frac{z^{4}-z^{6}}{w^{3}} d z, \frac{i\left(z^{4}+z^{6}\right)}{w^{3}} d z, \frac{z^{5}}{w^{3}} d z\right)$. Let $\left\{A_{j}, B_{j}\right\}_{j=1}^{3}$ be a canonical homology basis on $M$. We shall determine a complex $6 \times 6$ matrix given by

$$
\left(\int_{A_{1}}\left(\begin{array}{l}
G_{1} \\
G_{2}
\end{array}\right) \quad \int_{A_{2}}\left(\begin{array}{l}
G_{1} \\
G_{2}
\end{array}\right) \quad \int_{A_{3}}\left(\begin{array}{l}
G_{1} \\
G_{2}
\end{array}\right) \quad \int_{B_{1}}\left(\begin{array}{l}
G_{1} \\
G_{2}
\end{array}\right) \quad \int_{B_{2}}\left(\begin{array}{l}
G_{1} \\
G_{2}
\end{array}\right) \quad \int_{B_{3}}\left(\begin{array}{l}
G_{1} \\
G_{2}
\end{array}\right)\right) .
$$


Note that the upper complex $3 \times 6$ matrix

$$
\left(\int_{A_{1}} G_{1} \int_{A_{2}} G_{1} \int_{A_{3}} G_{1} \int_{B_{1}} G_{1} \int_{B_{2}} G_{1} \int_{B_{3}} G_{1}\right)
$$

is the complex period matrix on $M$.

Consider the following isometries on $M$ :

$$
\varphi_{1}(z, w)=\left(e^{\frac{2}{3} \pi i} z, e^{\frac{\pi}{3} i} w\right), \varphi_{2}(z, w)=\left(-\frac{1}{z}, \frac{w}{z^{4}}\right), j(z, w)=(z,-w) .
$$

It is straightforward to show that

$$
\varphi_{1}^{*}\left(\begin{array}{l}
G_{1} \\
G_{2}
\end{array}\right)=\left(\begin{array}{ccccccc}
\frac{1}{2} & \frac{\sqrt{3}}{2} & 0 & 0 & 0 & 0 \\
-\frac{\sqrt{3}}{2} & \frac{1}{2} & 0 & 0 & 0 & 0 \\
0 & 0 & -1 & 0 & 0 & 0 \\
0 & 0 & 0 & \frac{1}{2} & \frac{\sqrt{3}}{2} & 0
\end{array}\right)\left(\begin{array}{l}
G_{1} \\
G_{2}
\end{array}\right),
$$

Remark A1. For $\phi=0$, there exists an isometry defined by $\varphi_{3}(z, w)=(\bar{z}, \bar{w})$ and we find

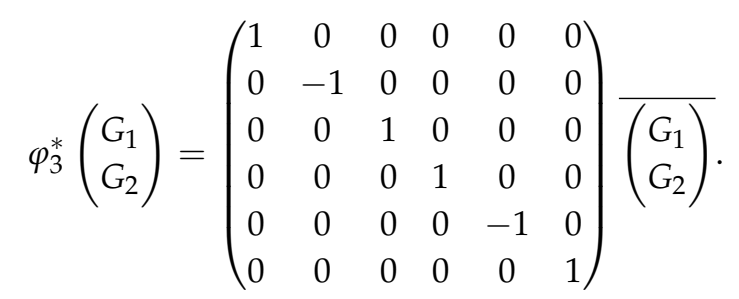

We now construct $M$ as a two-sheeted branched cover of $\overline{\mathbb{C}}$. Let $\pi: M \rightarrow \overline{\mathbb{C}}$ be the two-sheeted covering defined by $(z, w) \mapsto z$ which is branched at the following eight fixed points of $j$ :

$$
(0,0),(\infty, \infty),(a, 0),\left(a e^{\frac{2}{3} \pi i}, 0\right),\left(a e^{-\frac{2}{3} \pi i}, 0\right),\left(-\frac{1}{a}, 0\right),\left(\frac{e^{-\frac{\pi}{3} i}}{a}, 0\right),\left(\frac{e^{\frac{\pi}{3} i}}{a}, 0\right)
$$

We prepare two copies of $\overline{\mathbb{C}}$ and take two closed curves passing through the eight points, respectively. So we can divide $\overline{\mathbb{C}}$ into two domains and label " + " and " - " (see Figure A1). Slit them along the thick lines. Identifying each of the upper (resp. lower) edges of the thick lines in (i) with each of the lower (resp. upper) edges of the thick lines in (ii), we obtain the hyperelliptic Riemann surface $M$ of genus three. 

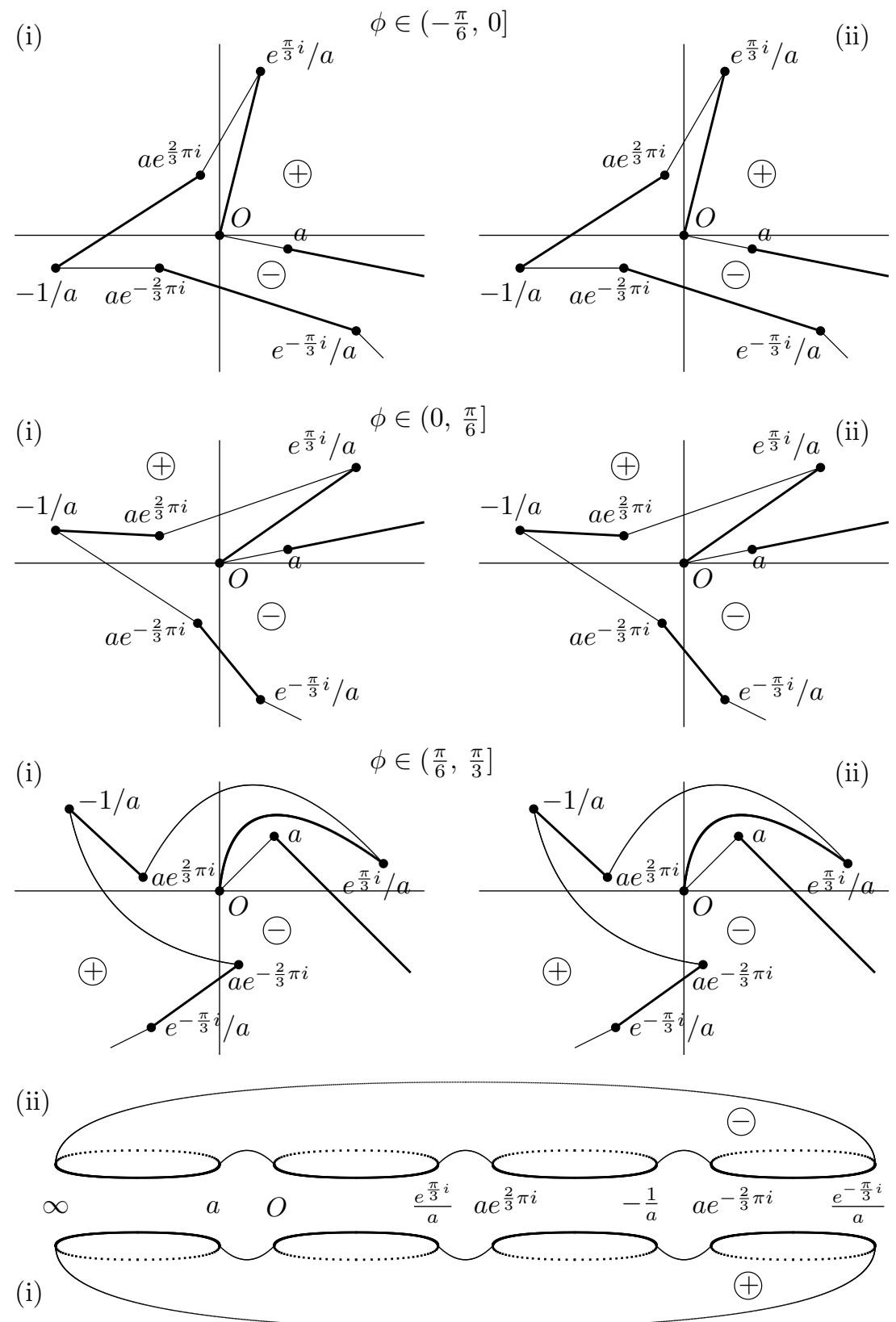

Figure A1. $M$ represented as a two-sheeted branched cover of $\overline{\mathbb{C}}$.

Remark that $j$ can be represented as the $180^{\circ}$ rotation around the middle axis between (i) and (ii) (see Figure A2).

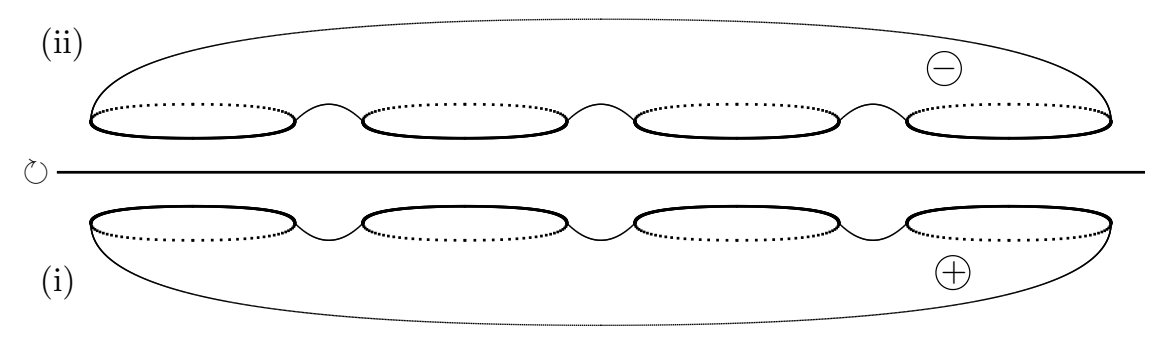

Figure A2. The action of $j$ on $M$. 


\section{Appendix A.1.1. A Canonical Homology Basis}

We first take the following three key paths.

$$
\begin{aligned}
& C_{1}=\left\{(z, w)=\left(e^{\frac{\pi}{3} i} t / a,-e^{\frac{\pi}{6} i} \sqrt{t\left(t^{3}-1\right)} \sqrt{a^{3}+t^{3} / a^{3}} / a^{2}\right) \mid 1 \leq t \leq \infty\right\}, \\
& C_{2}=\left\{(z, w)=\left(a t, i a^{2} \sqrt{t\left(1-t^{3}\right)} \sqrt{a^{3} t^{3}+1 / a^{3}}\right) \mid 0 \leq t \leq 1\right\}, \\
& C_{3}=\left\{(z, w)=\left(i t, \sqrt{t\left\{\left(1 / a^{3}-a^{3}\right) t^{3}-i\left(1+t^{6}\right)\right\}}\right) \mid 0 \leq t \leq \infty\right\},
\end{aligned}
$$

where we choose the branches as follows: $\sqrt{t\left(t^{3}-1\right)}>0$ and $\arg \sqrt{a^{3}+\frac{t^{3}}{a^{3}}} \in\left[-\frac{\pi}{2}, \frac{\pi}{4}\right]$ for $C_{1}$. $\sqrt{t\left(1-t^{3}\right)}>0$ and $\arg \sqrt{a^{3} t^{3}+\frac{1}{a^{3}}} \in\left[-\frac{\pi}{2}, \frac{\pi}{4}\right]$ for $C_{2}$. For $C_{3}$, arg $\sqrt{t\left\{\left(\frac{1}{a^{3}}-a^{3}\right) t^{3}-i\left(1+t^{6}\right)\right\}} \in$ $\left[-\frac{\pi}{2}, \frac{\pi}{4}\right]$.

Remark A2. Straightforward calculations yield

$$
\begin{aligned}
a^{3}+\frac{t^{3}}{a^{3}} & =\left(s^{3}+\frac{t^{3}}{s^{3}}\right) \cos (3 \phi)+i\left(s^{3}-\frac{t^{3}}{s^{3}}\right) \sin (3 \phi), \\
a^{3} t^{3}+\frac{1}{a^{3}} & =\left(s^{3} t^{3}+\frac{1}{s^{3}}\right) \cos (3 \phi)+i\left(s^{3} t^{3}-\frac{1}{s^{3}}\right) \sin (3 \phi), \\
\left(\frac{1}{a^{3}}-a^{3}\right) t^{3}-i\left(1+t^{6}\right) & =\left(\frac{1}{s^{3}}-s^{3}\right) t^{3} \cos (3 \phi)-i\left\{1+t^{6}+\left(\frac{1}{s^{3}}+s^{3}\right) t^{3} \sin (3 \phi)\right\} .
\end{aligned}
$$

For $C_{1}$, we have $\arg \left(a^{3}+\frac{t^{3}}{a^{3}}\right) \in\left[-\pi, \frac{\pi}{2}\right]$. For $C_{2}, \arg \left(a^{3} t^{3}+\frac{1}{a^{3}}\right) \in\left[-\pi, \frac{\pi}{2}\right]$ holds. For $C_{3}$, we find $\arg \left\{\left(\frac{1}{a^{3}}-a^{3}\right) t^{3}-i\left(1+t^{6}\right)\right\} \in\left[-\pi, \frac{\pi}{2}\right]$. Hence $\sqrt{a^{3}+\frac{t^{3}}{a^{3}}}, \sqrt{a^{3} t^{3}+\frac{1}{a^{3}}}, \sqrt{\left(\frac{1}{a^{3}}-a^{3}\right) t^{3}-i\left(1+t^{6}\right)}$ have single-valued branches for $\phi \in\left(-\frac{\pi}{6}, \frac{\pi}{3}\right]$.

We now consider two cases, namely, the case $\phi \in\left(-\frac{\pi}{6}, \frac{\pi}{6}\right)$ and the case $\phi \in\left[\frac{\pi}{6}, \frac{\pi}{3}\right]$. We assume $\phi \in\left(-\frac{\pi}{6}, \frac{\pi}{6}\right)$, and introduce the following two paths.

$$
\begin{aligned}
& C_{4}=\left\{(z, w)=\left(e^{\frac{\pi}{3} i} t / a, i e^{\frac{\pi}{6} i} \sqrt{t\left(1-t^{3}\right)} \sqrt{a^{3}+t^{3} / a^{3}} / a^{2}\right) \mid 0 \leq t \leq 1\right\} \\
& C_{5}=\left\{(z, w)=\left(a t, a^{2} \sqrt{t\left(t^{3}-1\right)} \sqrt{a^{3} t^{3}+1 / a^{3}}\right) \mid 1 \leq t \leq \infty\right\}
\end{aligned}
$$

where we choose the branches as follows: $\sqrt{t\left(1-t^{3}\right)}>0$ and arg $\sqrt{a^{3}+\frac{t^{3}}{a^{3}}} \in\left[-\frac{\pi}{4}, \frac{\pi}{4}\right]$ for $C_{4}$. $\sqrt{t\left(t^{3}-1\right)}>0$ and $\arg \sqrt{a^{3} t^{3}+\frac{1}{a^{3}}} \in\left[-\frac{\pi}{4}, \frac{\pi}{4}\right]$ for $C_{5}$.

Using $C_{4}$ and $C_{5}$, we shall describe a canonical homology basis on $M$ by $\left\{C_{j}\right\}_{j=1}^{3}$. To start with, let us choose $C_{4}$ as in Figure A3. 
(i)
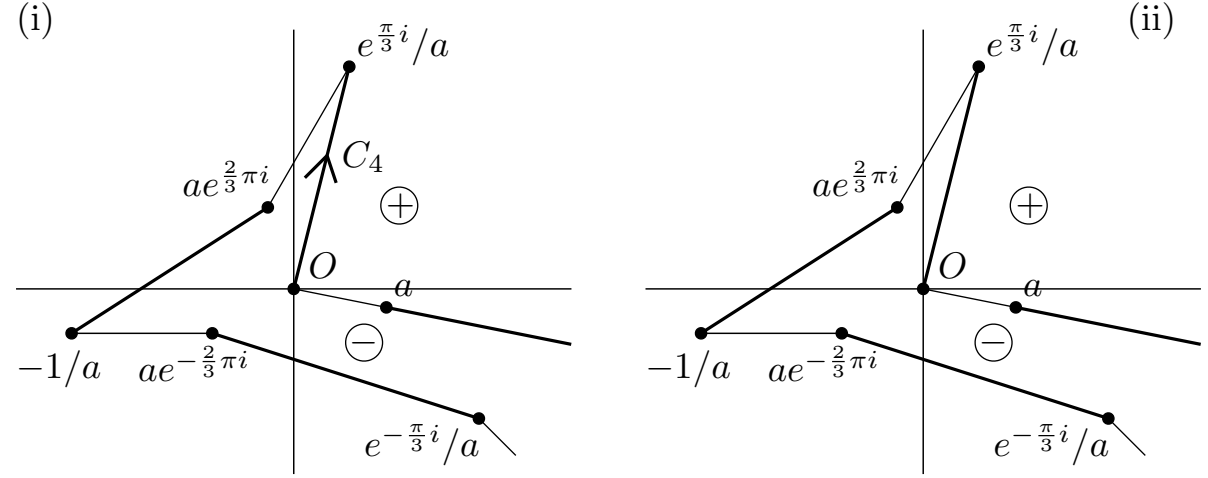

(i)

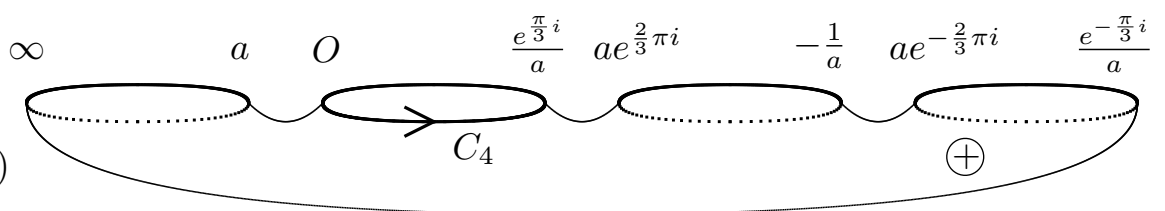

Figure A3. $C_{4}$ in (i).

There exist $l, m, n \in\{0,1\}$ such that $j^{l}\left(C_{1}\right)+C_{4}$ is homologous to $j^{m}\left(C_{2}\right)+j^{n}\left(C_{5}\right)$ (write $j^{l}\left(C_{1}\right)+$ $\left.C_{4} \sim j^{m}\left(C_{2}\right)+j^{n}\left(C_{5}\right)\right)$ for an arbitrary $\phi \in\left(-\frac{\pi}{6}, \frac{\pi}{6}\right)$. Setting $\phi=0$, we have $l=m=n=0$ (see $\S 5.2 .1$ in [8]). Thus, $l=m=n=0$ hold for $\phi \in\left(-\frac{\pi}{6}, \frac{\pi}{6}\right)$ (see Figure A4). In the process we have also shown that the equation

$$
\begin{aligned}
& \sqrt{3} \int_{0}^{1} \frac{a^{2}-t^{2}}{\sqrt{t\left(1-t^{3}\right)} \sqrt{a^{3}+\frac{t^{3}}{a^{3}}}} d t-i \int_{0}^{1} \frac{a^{2}+t^{2}}{\sqrt{t\left(1-t^{3}\right)} \sqrt{a^{3}+\frac{t^{3}}{a^{3}}}} d t \\
& \quad+\sqrt{3} i \int_{0}^{1} \frac{1-a^{2} t^{2}}{\sqrt{t\left(1-t^{3}\right)} \sqrt{a^{3} t^{3}+\frac{1}{a^{3}}}} d t-\int_{0}^{1} \frac{1+a^{2} t^{2}}{\sqrt{t\left(1-t^{3}\right)} \sqrt{a^{3} t^{3}+\frac{1}{a^{3}}}} d t=0
\end{aligned}
$$

is satisfied for $\phi \in\left(-\frac{\pi}{6}, \frac{\pi}{6}\right)$.

By choosing a suitable $l \in\{0,1\}$, we find $C_{1}+j\left(C_{4}\right) \sim j^{l}\left(C_{3}\right)$. From $\int_{C_{1}+j\left(C_{4}\right)} \frac{2 z}{w} d z=\int_{j^{l}\left(C_{3}\right)} \frac{2 z}{w} d z$, we have

$$
\int_{0}^{1} \frac{t}{\sqrt{t\left(1-t^{3}\right)} \sqrt{a^{3}+\frac{t^{3}}{a^{3}}}} d t+i \int_{0}^{1} \frac{t}{\sqrt{t\left(1-t^{3}\right)} \sqrt{a^{3} t^{3}+\frac{1}{a^{3}}}} d t=(-1)^{l} \int_{0}^{\infty} \frac{t}{\sqrt{t\left\{\left(\frac{1}{a^{3}}-a^{3}\right) t^{3}-i\left(1+t^{6}\right)\right\}}} d t .
$$

Setting $\phi=0$, we obtain $l=0$. It follows that $C_{1}+j\left(C_{4}\right) \sim C_{3}$ holds for $\phi \in\left(-\frac{\pi}{6}, \frac{\pi}{6}\right)$ (see Figure A5). 
(i)
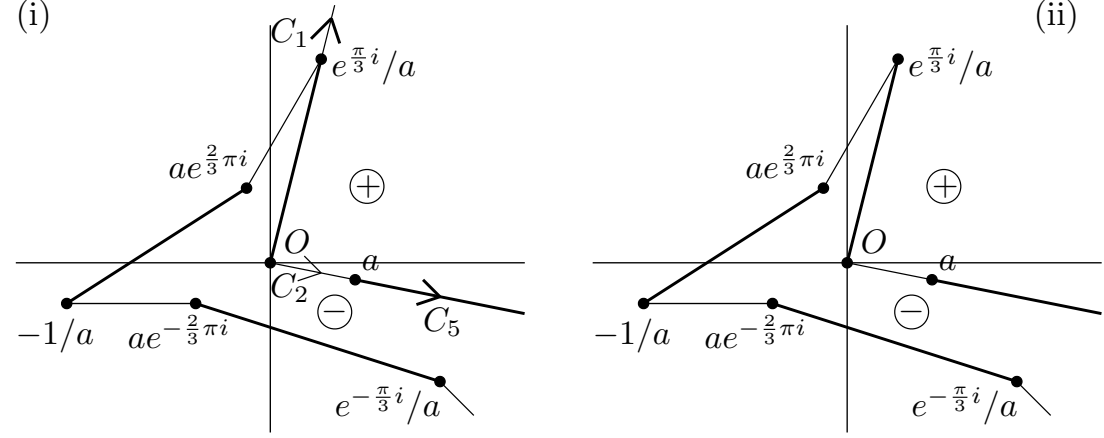

(i)

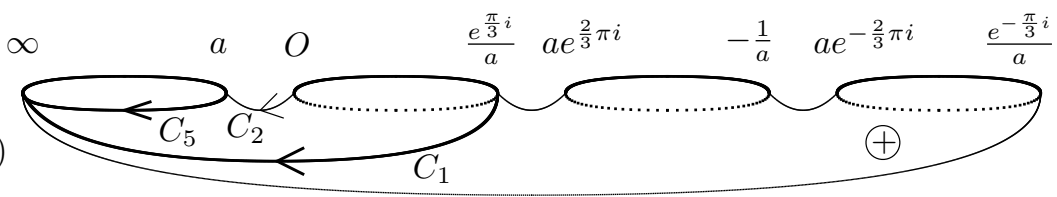

Figure A4. $C_{1}, C_{2}, C_{5}$ in (i).

(i)
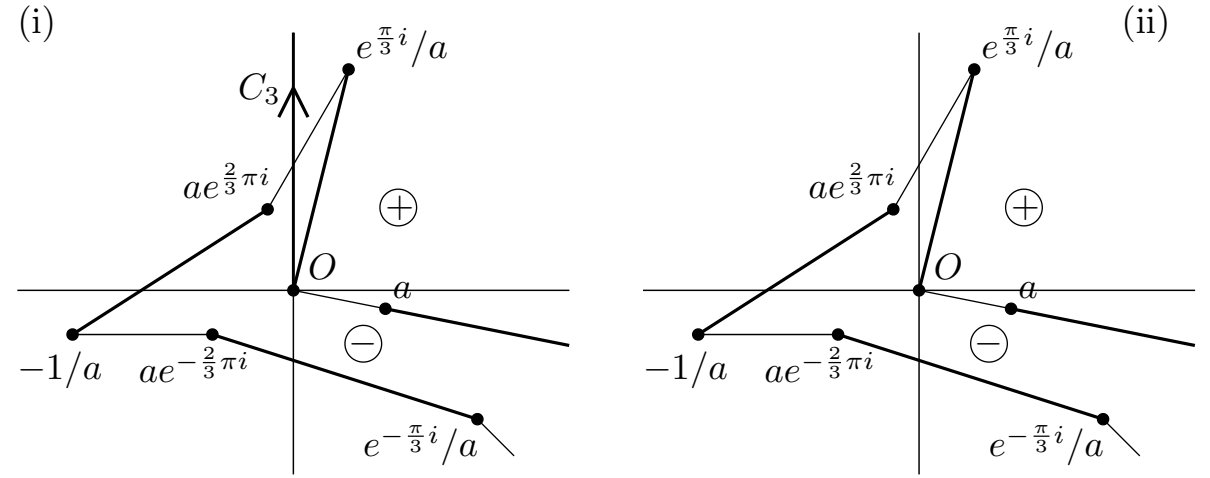

(i)

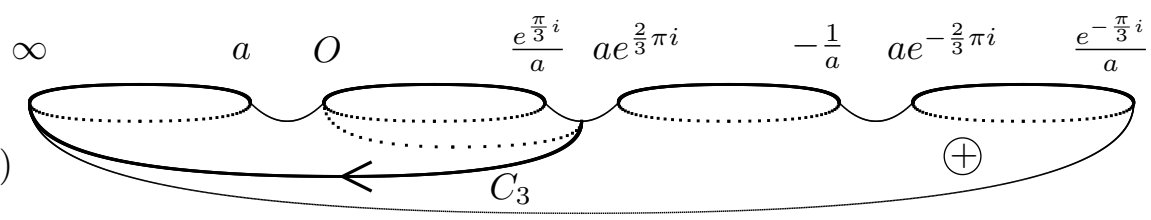

Figure A5. $C_{3}$ in (i).

By $\int_{C_{1}+j\left(C_{4}\right)} \frac{1-z^{2}}{w} d z=\int_{C_{3}} \frac{1-z^{2}}{w} d z, \int_{C_{1}+j\left(C_{4}\right)} \frac{i\left(1+z^{2}\right)}{w} d z=\int_{C_{3}} \frac{i\left(1+z^{2}\right)}{w} d z$, and (A2), we find

$$
\begin{aligned}
& 2 i \int_{0}^{1} \frac{1+t^{2}}{\sqrt{t\left\{\left(\frac{1}{a^{3}}-a^{3}\right) t^{3}-i\left(1+t^{6}\right)\right\}}} d t \\
&= \frac{1}{a}\left\{-\frac{1}{2} \int_{0}^{1} \frac{a^{2}-t^{2}}{\sqrt{t\left(1-t^{3}\right)} \sqrt{a^{3}+\frac{t^{3}}{a^{3}}}} d t+\frac{\sqrt{3}}{2} i \int_{0}^{1} \frac{a^{2}+t^{2}}{\sqrt{t\left(1-t^{3}\right)} \sqrt{a^{3}+\frac{t^{3}}{a^{3}}}} d t\right. \\
&\left.\quad+\frac{i}{2} \int_{0}^{1} \frac{1-a^{2} t^{2}}{\sqrt{t\left(1-t^{3}\right)} \sqrt{a^{3} t^{3}+\frac{1}{a^{3}}}} d t-\frac{\sqrt{3}}{2} \int_{0}^{1} \frac{1+a^{2} t^{2}}{\sqrt{t\left(1-t^{3}\right)} \sqrt{a^{3} t^{3}+\frac{1}{a^{3}}}} d t\right\}, \\
&-i \int_{0}^{1} \frac{a^{2}+t^{2}}{\sqrt{t\left(1-t^{3}\right)} \sqrt{a^{3}+\frac{t^{3}}{a^{3}}}} d t-\sqrt{3} \int_{0}^{1} \frac{a^{2}-t^{2}}{\sqrt{t\left(1-t^{3}\right)} \sqrt{a^{3}+\frac{t^{3}}{a^{3}}}} d t \\
&+\int_{0}^{1} \frac{1+a^{2} t^{2}}{\sqrt{t\left(1-t^{3}\right)} \sqrt{a^{3} t^{3}+\frac{1}{a^{3}}}} d t+\sqrt{3} i \int_{0}^{1} \frac{1-a^{2} t^{2}}{\sqrt{t\left(1-t^{3}\right)} \sqrt{a^{3} t^{3}+\frac{1}{a^{3}}}} d t=0,
\end{aligned}
$$




$$
\begin{aligned}
\int_{0}^{\infty} & \frac{t}{\sqrt{t\left\{\left(\frac{1}{a^{3}}-a^{3}\right) t^{3}-i\left(1+t^{6}\right)\right\}}} d t \\
& =\int_{0}^{1} \frac{t}{\sqrt{t\left(1-t^{3}\right)} \sqrt{a^{3}+\frac{t^{3}}{a^{3}}}} d t+i \int_{0}^{1} \frac{t}{\sqrt{t\left(1-t^{3}\right)} \sqrt{a^{3} t^{3}+\frac{1}{a^{3}}}} d t
\end{aligned}
$$

for $\phi \in\left(-\frac{\pi}{6}, \frac{\pi}{6}\right)$. Combining (A1) and (A4), we have

$$
\begin{aligned}
\sqrt{3} \int_{0}^{1} \frac{a^{2}-t^{2}}{\sqrt{t\left(1-t^{3}\right)} \sqrt{a^{3}+\frac{t^{3}}{a^{3}}}} d t & =\int_{0}^{1} \frac{1+a^{2} t^{2}}{\sqrt{t\left(1-t^{3}\right)} \sqrt{a^{3} t^{3}+\frac{1}{a^{3}}}} d t \\
\sqrt{3} \int_{0}^{1} \frac{1-a^{2} t^{2}}{\sqrt{t\left(1-t^{3}\right)} \sqrt{a^{3} t^{3}+\frac{1}{a^{3}}}} d t & =\int_{0}^{1} \frac{a^{2}+t^{2}}{\sqrt{t\left(1-t^{3}\right)} \sqrt{a^{3}+\frac{t^{3}}{a^{3}}}} d t
\end{aligned}
$$

for $\phi \in\left(-\frac{\pi}{6}, \frac{\pi}{6}\right)$.

Similarly, there exists $l \in\{0,1\}$ such that $C_{2}+j\left(C_{5}\right) \sim j^{l}\left(\varphi_{1}^{2}\left(C_{3}\right)\right)$. From $\int_{C_{2}+j\left(C_{5}\right)} \frac{2 z}{w} d z=\int_{j^{l}}\left(\varphi_{1}^{2}\left(C_{3}\right)\right) \frac{2 z}{w} d z$ and (A5), we find $l=0$ (see Figure A6).

(i)
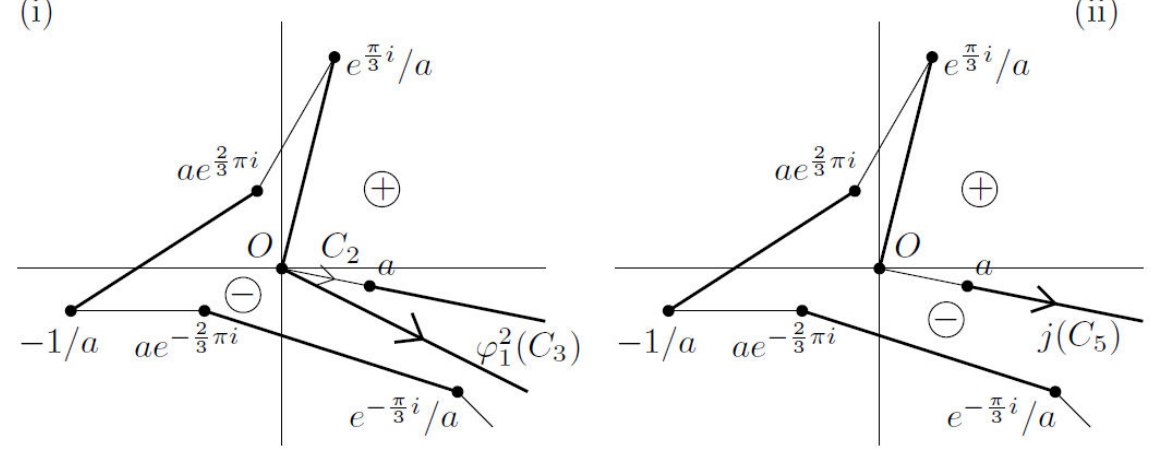

(i)

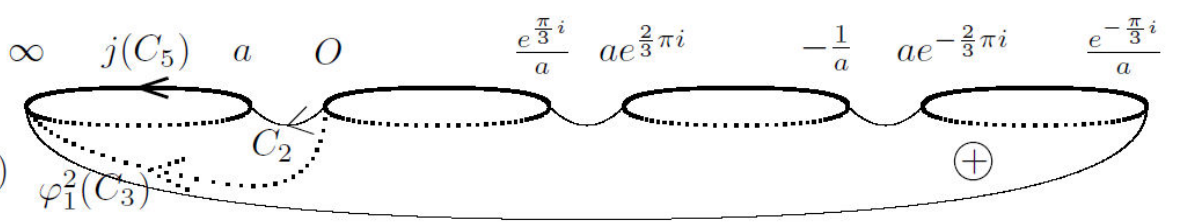

Figure A6. $C_{2}, \varphi_{1}^{2}\left(C_{3}\right), j\left(C_{5}\right)$ in (i). (i)

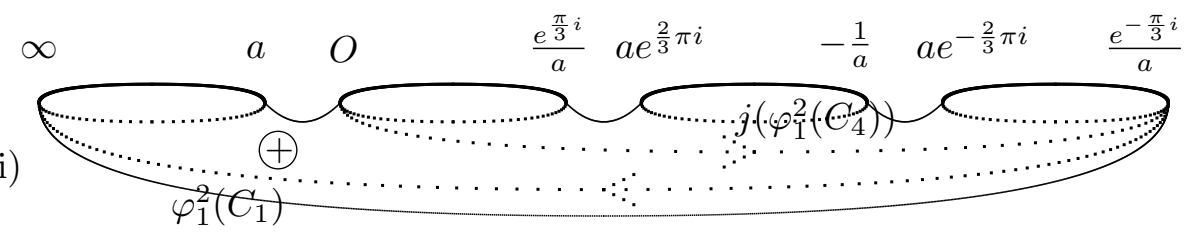

Figure A7. $\varphi_{1}^{2}\left(C_{1}\right), j\left(\varphi_{1}^{2}\left(C_{4}\right)\right)$ in (i).

Choosing suitable $l, m \in\{0,1\}$, we have $j^{l}\left(\varphi_{1}\left(C_{2}\right)\right)+j^{m}\left(\varphi_{1}\left(C_{5}\right)\right) \sim C_{3}$. From $\int_{j^{l}}\left(\varphi_{1}\left(C_{2}\right)\right)+j^{m}\left(\varphi_{1}\left(C_{5}\right)\right) \frac{2 z}{w} d z=\int_{C_{3}} \frac{2 z}{w} d z$ and (A5), we find $l=1, m=0$ (see Figure A8). 
(i)

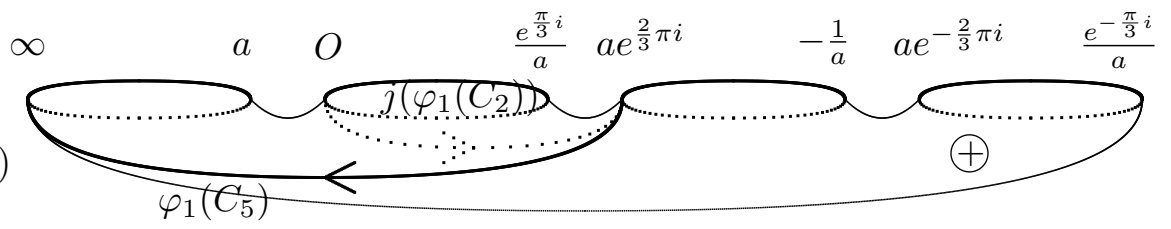

Figure A8. $j\left(\varphi_{1}\left(C_{2}\right)\right), \varphi_{1}\left(C_{5}\right)$ in (i).

To determine $\varphi_{1}\left(C_{3}\right)$ and $j\left(\varphi_{1}\left(C_{3}\right)\right)$, we introduce the following new path.

$$
C_{6}:=\left\{(z, w)=\left(e^{i t},-e^{2 i t} \sqrt{2 i \sin (3 t)+1 / a^{3}-a^{3}}\right) \mid \pi / 2 \leq t \leq 7 \pi / 6\right\},
$$

where we choose the branch such that $\arg \sqrt{2 i \sin (3 t)+1 / a^{3}-a^{3}} \in(-\pi / 4, \pi / 4)$.

Remark A3. For $C_{6}$, we obtain

$$
\frac{w^{2}}{z^{4}}=z^{3}-\frac{1}{z^{3}}+\frac{1}{a^{3}}-a^{3}=\left(\frac{1}{s^{3}}-s^{3}\right) \cos (3 \phi)+i\left\{2 \sin (3 t)-\left(\frac{1}{s^{3}}+s^{3}\right) \sin (3 \phi)\right\} .
$$

Thus, $\arg \frac{w^{2}}{z^{4}} \in\left(-\frac{\pi}{2}, \frac{\pi}{2}\right)$ holds for $\phi \in\left(-\frac{\pi}{6}, \frac{\pi}{6}\right)$ and we may choose the branch such that $\arg \frac{w}{z^{2}} \in\left(\frac{3}{4} \pi, \frac{5}{4} \pi\right)$. Substituting $t=\pi / 2$, we have $w=\sqrt{1 / a^{3}-a^{3}-2 i}$, where $\arg \sqrt{1 / a^{3}-a^{3}-2 i} \in(-\pi / 4, \pi / 4)$.

By Remark A3, $C_{3} \cap C_{6} \neq \varnothing$ holds. Substituting $t=\frac{7}{6} \pi$ to $(z, w)$ in $C_{6}$, we find $(z, w)=\left(i e^{\frac{2}{3} \pi i},-e^{\frac{\pi}{3} i} \sqrt{\frac{1}{a^{3}}-a^{3}-2 i}\right)=j\left(\varphi_{1}\left(\left(i, \sqrt{\frac{1}{a^{3}}-a^{3}-2 i}\right)\right)\right) \in j\left(\varphi_{1}\left(C_{3}\right)\right)$. Hence $j\left(\varphi_{1}\left(C_{3}\right)\right) \cap C_{6} \neq$ (see Figures A9 and A10).
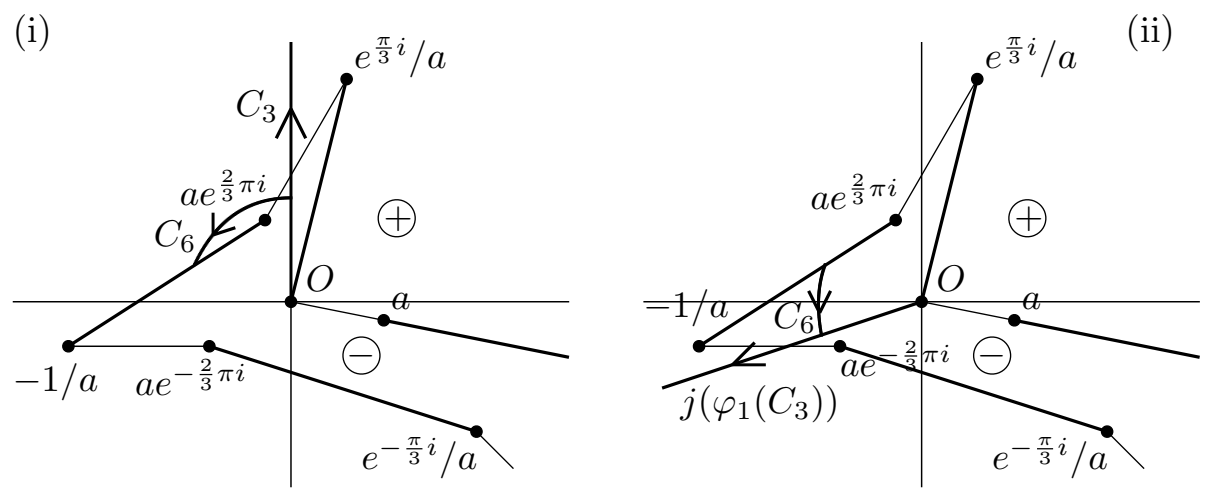

(ii)

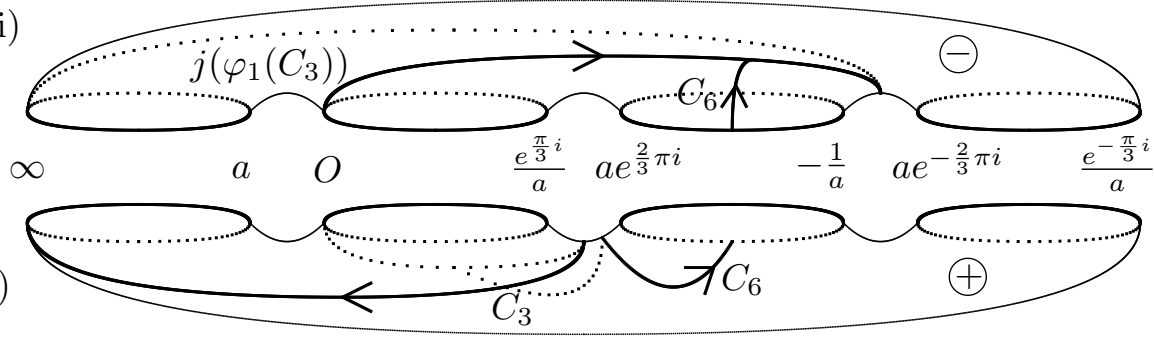

Figure A9. $C_{6}$ in $M$. 
(ii)

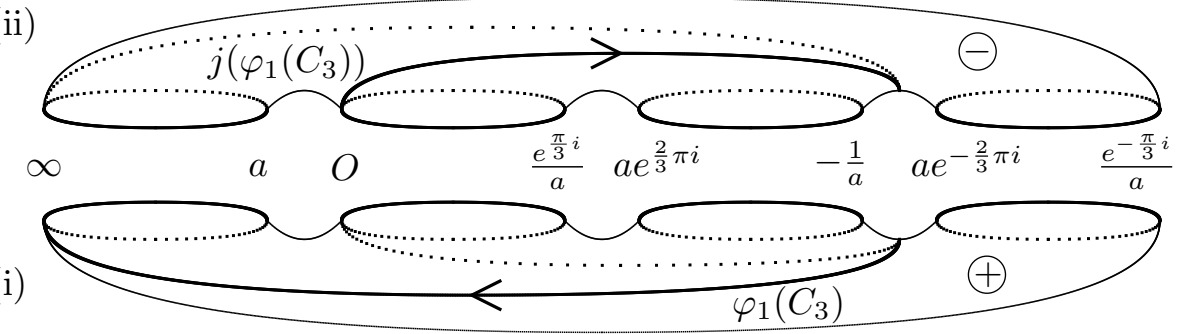

Figure A10. $\varphi_{1}\left(C_{3}\right), j\left(\varphi_{1}\left(C_{3}\right)\right)$ in $M$.

By $C_{1}+j\left(C_{4}\right) \sim C_{3}$, we obtain $\varphi_{1}\left(C_{1}\right)+j\left(\varphi_{1}\left(C_{4}\right)\right) \sim \varphi_{1}\left(C_{3}\right)$, and so Figure A11 follows.

(i)

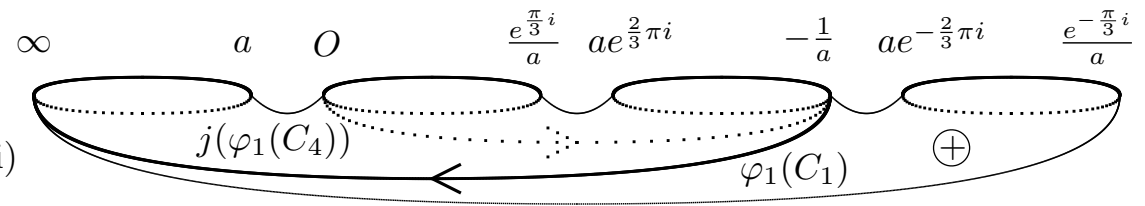

Figure A11. $\varphi_{1}\left(C_{1}\right), j\left(\varphi_{1}\left(C_{4}\right)\right)$ in (i).

From $j\left(\varphi_{1}\left(C_{2}\right)\right)+\varphi_{1}\left(C_{5}\right) \sim C_{3}$, we have $j\left(\varphi_{1}^{2}\left(C_{2}\right)\right)+\varphi_{1}^{2}\left(C_{5}\right) \sim \varphi_{1}\left(C_{3}\right)$ (see Figure A12).

(i)

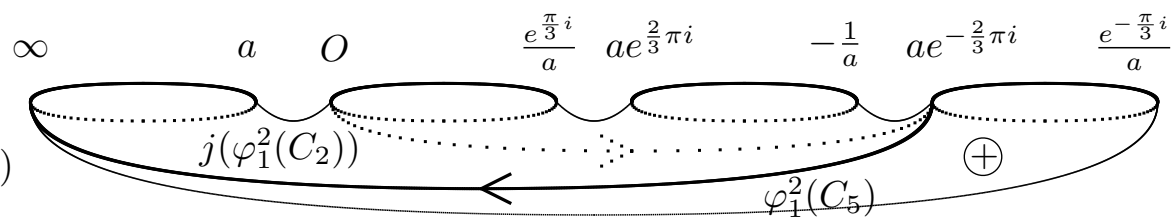

Figure A12. $j\left(\varphi_{1}^{2}\left(C_{2}\right)\right), \varphi_{1}^{2}\left(C_{5}\right)$ in (i).

Therefore, we can describe a canonical homology basis as follows (see Figure A13).

$$
\begin{aligned}
& A_{1}=-C_{2}+j\left(C_{2}\right), A_{2}=C_{1}-j\left(C_{1}\right)-\varphi_{1}\left(C_{2}\right)+j\left(\varphi_{1}\left(C_{2}\right)\right)-C_{3}+j\left(C_{3}\right), \\
& A_{3}=-\varphi_{1}^{2}\left(C_{1}\right)+j\left(\varphi_{1}^{2}\left(C_{1}\right)\right)-A_{1}-A_{2}, B_{1}=-C_{1}+j\left(C_{1}\right)+\varphi_{1}^{2}\left(C_{3}\right)-j\left(C_{3}\right), \\
& B_{2}=-C_{3}+\varphi_{1}^{2}\left(C_{3}\right), B_{3}=-\varphi_{1}\left(C_{3}\right)+\varphi_{1}^{2}\left(C_{3}\right) .
\end{aligned}
$$

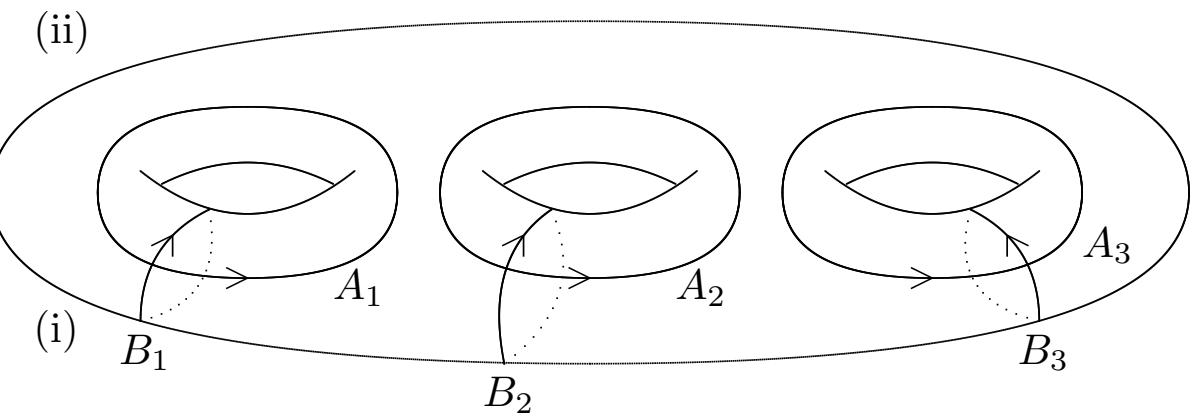

Figure A13. A canonical homology basis on $M$.

Next we assume $\phi \in\left[\frac{\pi}{6}, \frac{\pi}{3}\right]$, and introduce the following two paths.

$$
\begin{aligned}
& C_{7}=\left\{(z, w)=\left(e^{\frac{\pi}{3} i} t / a, i e^{\frac{\pi}{6} i} \sqrt{t\left(1-t^{3}\right)} \sqrt{a^{3}+t^{3} / a^{3}} / a^{2}\right) \mid 0 \leq t \leq 1\right\}, \\
& C_{8}=\left\{(z, w)=\left(a t, a^{2} \sqrt{t\left(t^{3}-1\right)} \sqrt{a^{3} t^{3}+1 / a^{3}}\right) \mid 1 \leq t \leq \infty\right\}
\end{aligned}
$$


where we choose the branches as follows: $\sqrt{t\left(1-t^{3}\right)}>0$ and $\arg \sqrt{a^{3}+\frac{t^{3}}{a^{3}}} \in\left[-\frac{3}{4} \pi,-\frac{\pi}{4}\right]$ for $C_{7}$. $\sqrt{t\left(t^{3}-1\right)}>0$ and $\arg \sqrt{a^{3} t^{3}+\frac{1}{a^{3}}} \in\left[-\frac{3}{4} \pi,-\frac{\pi}{4}\right]$ for $C_{8}$.

Using $C_{7}$ and $C_{8}$, we shall describe a canonical homology basis on $M$ by $\left\{C_{j}\right\}_{j=1}^{3}$. To start with, let us choose $C_{3}$ as in Figure A14.
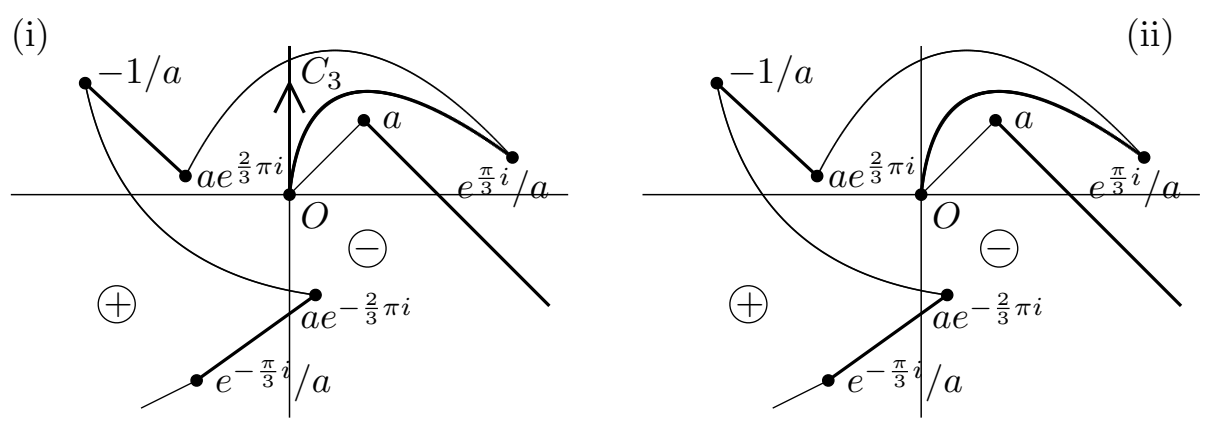

(i)

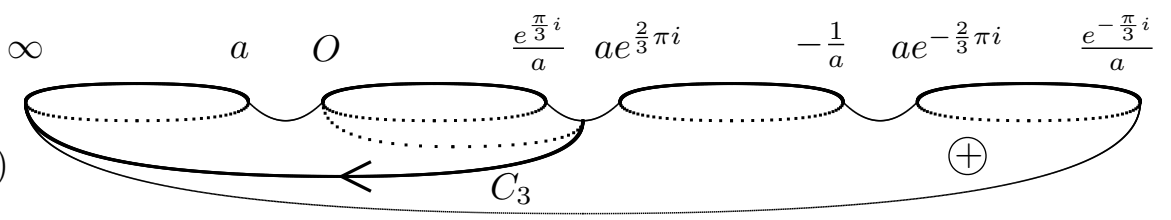

Figure A14. $C_{3}$ in (i).

There exist $l, m \in\{0,1\}$ such that $j^{l}\left(C_{2}\right)+j^{m}\left(C_{8}\right) \sim C_{3}$. So we find

$$
\int_{j^{l}\left(C_{2}\right)+j^{m}\left(C_{8}\right)} \frac{2 z}{w} d z=\int_{C_{3}} \frac{2 z}{w} d z
$$

that is,

$$
\begin{aligned}
\int_{0}^{\infty} & \frac{t}{\sqrt{t\left\{\left(\frac{1}{a^{3}}-a^{3}\right) t^{3}-i\left(1+t^{6}\right)\right\}}} d t \\
& =(-1)^{l} i \int_{0}^{1} \frac{t}{\sqrt{t\left(1-t^{3}\right)} \sqrt{a^{3} t^{3}+\frac{1}{a^{3}}}} d t+(-1)^{m+1} \int_{0}^{1} \frac{t}{\sqrt{t\left(1-t^{3}\right)} \sqrt{a^{3}+\frac{t^{3}}{a^{3}}}} d t
\end{aligned}
$$

Setting $\phi=\frac{\pi}{3}$, we have $l=m=1$ (see Figure A15).

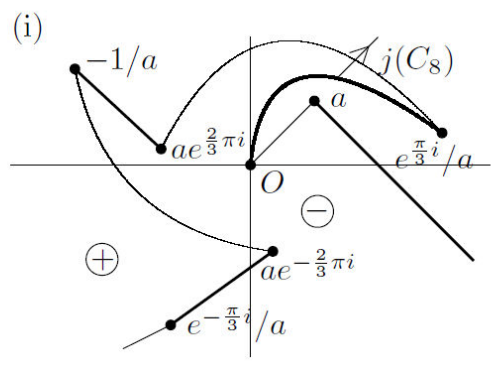

(ii)

Figure A15. Cont. 
(ii)

(i)

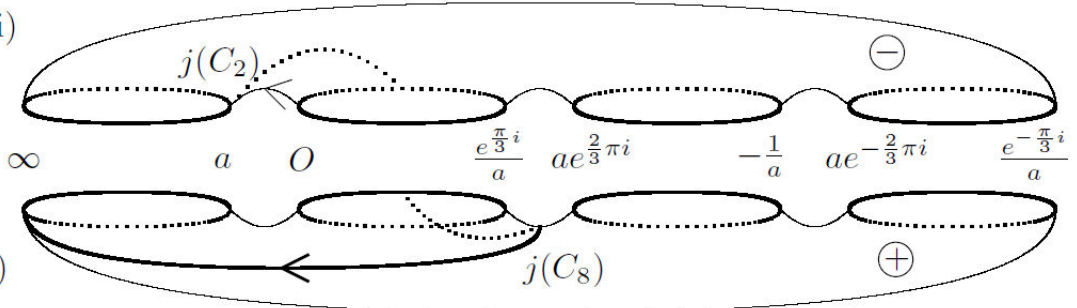

Figure A15. $j\left(C_{2}\right), j\left(C_{8}\right)$ in $M$.

Thus, we obtain

$\int_{0}^{\infty} \frac{t}{\sqrt{t\left\{\left(\frac{1}{a^{3}}-a^{3}\right) t^{3}-i\left(1+t^{6}\right)\right\}}} d t=\int_{0}^{1} \frac{t}{\sqrt{t\left(1-t^{3}\right)} \sqrt{a^{3}+\frac{t^{3}}{a^{3}}}} d t-i \int_{0}^{1} \frac{t}{\sqrt{t\left(1-t^{3}\right)} \sqrt{a^{3} t^{3}+\frac{1}{a^{3}}}} d t$.

By $\int_{j\left(C_{2}\right)+j\left(C_{8}\right)} \frac{1-z^{2}}{w} d z=\int_{C_{3}} \frac{1-z^{2}}{w} d z$, we find

$$
2 \int_{0}^{1} \frac{1+t^{2}}{\sqrt{t\left\{\left(\frac{1}{a^{3}}-a^{3}\right) t^{3}-i\left(1+t^{6}\right)\right\}}} d t=\frac{1}{a}\left\{\int_{0}^{1} \frac{1-a^{2} t^{2}}{\sqrt{t\left(1-t^{3}\right)} \sqrt{a^{3} t^{3}+\frac{1}{a^{3}}}} d t-i \int_{0}^{1} \frac{a^{2}-t^{2}}{\sqrt{t\left(1-t^{3}\right)} \sqrt{a^{3}+\frac{t^{3}}{a^{3}}}} d t .\right.
$$

From $\int_{j\left(C_{2}\right)+j\left(C_{8}\right)} \frac{i\left(1+z^{2}\right)}{w} d z=\int_{C_{3}} \frac{i\left(1+z^{2}\right)}{w} d z$, we have

$$
\int_{0}^{1} \frac{1+a^{2} t^{2}}{\sqrt{t\left(1-t^{3}\right)} \sqrt{a^{3} t^{3}+\frac{1}{a^{3}}}} d t=-i \int_{0}^{1} \frac{a^{2}+t^{2}}{\sqrt{t\left(1-t^{3}\right)} \sqrt{a^{3}+\frac{t^{3}}{a^{3}}}} d t
$$

To determine $j\left(\varphi_{1}\left(C_{3}\right)\right)$, we introduce the following path.

$$
C_{9}=\left\{(z, w)=\left(e^{i t},-e^{2 i t} \sqrt{2 i \sin (3 t)+1 / a^{3}-a^{3}}\right) \mid \pi / 2 \leq t \leq 7 \pi / 6\right\},
$$

where we choose the branch such that $\arg \sqrt{2 i \sin (3 t)+1 / a^{3}-a^{3}} \in[-3 \pi / 4,-\pi / 4]$.

Remark A4. For $C_{9}$, we obtain

$$
\frac{w^{2}}{z^{4}}=\left(\frac{1}{s^{3}}-s^{3}\right) \cos (3 \phi)+i\left\{2 \sin (3 t)-\left(\frac{1}{s^{3}}+s^{3}\right) \sin (3 \phi)\right\} .
$$

Thus, $\arg \frac{w^{2}}{z^{4}} \in\left[-\frac{3}{2} \pi,-\frac{\pi}{2}\right]$ holds for $\phi \in\left[\frac{\pi}{6}, \frac{\pi}{3}\right]$ and we may choose the branch such that $\arg \frac{w}{z^{2}} \in\left[\frac{\pi}{4}, \frac{3}{4} \pi\right]$. Substituting $t=\pi / 2$, we have $w=\sqrt{1 / a^{3}-a^{3}-2 i}$, where $\arg \sqrt{1 / a^{3}-a^{3}-2 i} \in$ $[-3 \pi / 4,-\pi / 4]$.

By Remark A4, $C_{3} \cap C_{9} \neq \varnothing$ holds. Substituting $t=\frac{7}{6} \pi$ to $(z, w)$ in $C_{9}$, we find $(z, w)=\left(i e^{\frac{2}{3} \pi i},-e^{\frac{\pi}{3} i} \sqrt{\frac{1}{a^{3}}-a^{3}-2 i}\right)=j\left(\varphi_{1}\left(\left(i, \sqrt{\frac{1}{a^{3}}-a^{3}-2 i}\right)\right)\right) \in j\left(\varphi_{1}\left(C_{3}\right)\right)$. Hence $j\left(\varphi_{1}\left(C_{3}\right)\right) \cap C_{9} \neq \varnothing$ (see Figure A16). 
(i)

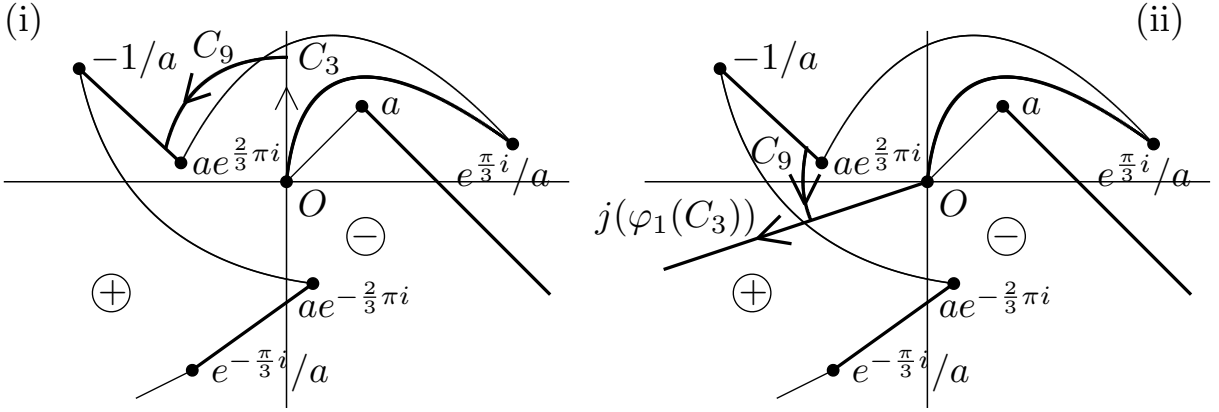

(ii)

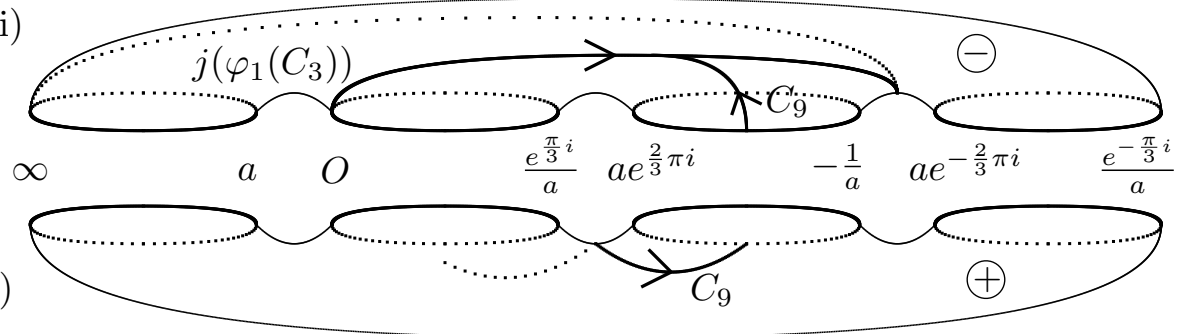

Figure A16. $j\left(\varphi_{1}\left(C_{3}\right)\right), C_{9}$ in $M$.

From $j\left(C_{2}\right)+j\left(C_{8}\right) \sim C_{3}$, we have $\varphi_{1}\left(C_{2}\right)+\varphi_{1}\left(C_{8}\right) \sim j\left(\varphi_{1}\left(C_{3}\right)\right)$ (see Figure A17).

(i)
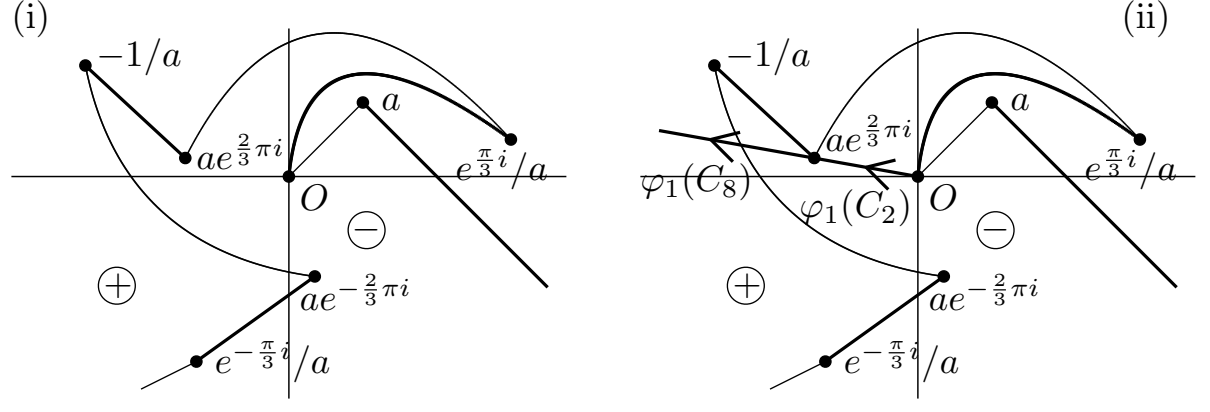

(ii)

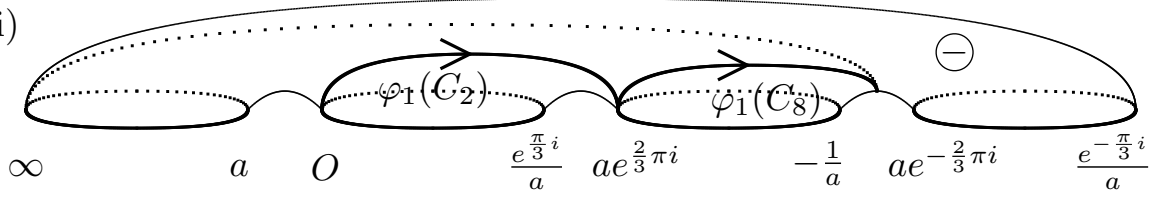

Figure A17. $\varphi_{1}\left(C_{2}\right), \varphi_{1}\left(C_{8}\right)$ in (ii).

Choosing suitable $l, m \in\{0,1\}$, we obtain $j^{l}\left(\varphi_{1}^{2}\left(C_{1}\right)\right)+j^{m}\left(\varphi_{1}^{2}\left(C_{7}\right)\right) \sim j\left(\varphi_{1}\left(C_{3}\right)\right)$. By $\int_{j^{l}}\left(\varphi_{1}^{2}\left(C_{1}\right)\right)+j^{m}\left(\varphi_{1}^{2}\left(C_{7}\right)\right) \frac{2 z}{w} d z=\int_{j\left(\varphi_{1}\left(C_{3}\right)\right)} \frac{2 z}{w} d z$ and (A8), we find $l=m=1$ (see Figure A20). 
(i)

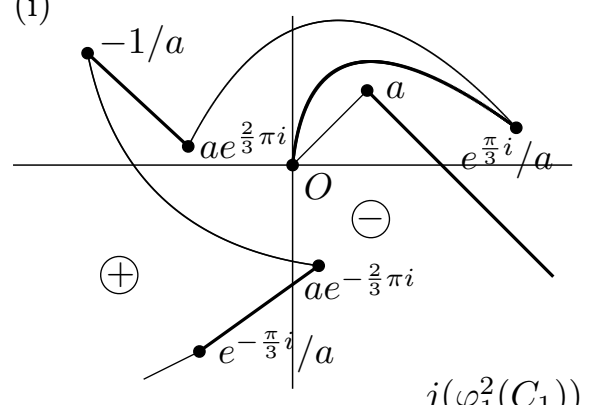

(ii)

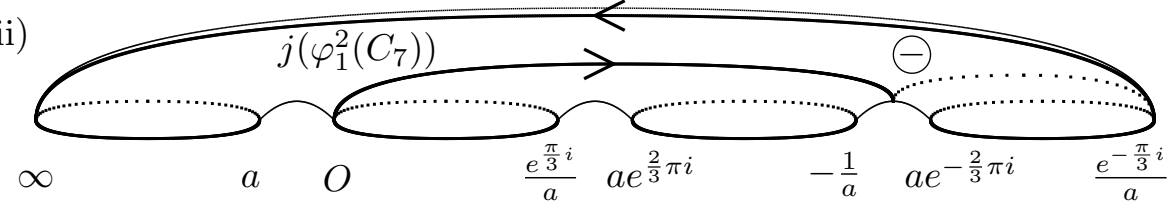

(ii)

Figure A18. $j\left(\varphi_{1}^{2}\left(C_{1}\right)\right), j\left(\varphi_{1}^{2}\left(C_{7}\right)\right)$ in (ii).

By similar arguments as $j\left(\varphi_{1}\left(C_{3}\right)\right) \cap C_{9} \neq \varnothing$, we obtain $j\left(\varphi_{1}\left(C_{3}\right)\right) \cap j\left(\varphi_{1}\left(C_{9}\right)\right) \neq \varnothing$ and $\varphi_{1}^{2}\left(C_{3}\right) \cap j\left(\varphi_{1}\left(C_{9}\right)\right) \neq \varnothing$ (see Figure A19).

(i)
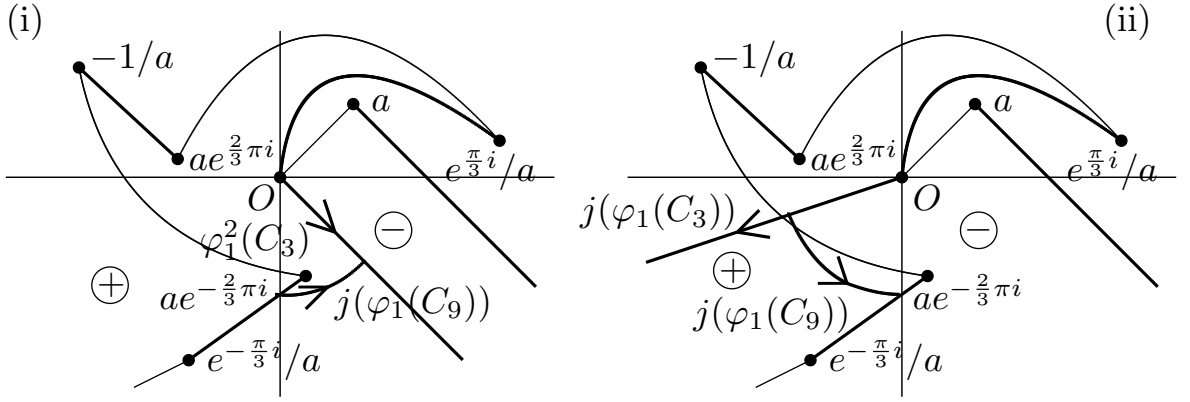

(ii)

(i)

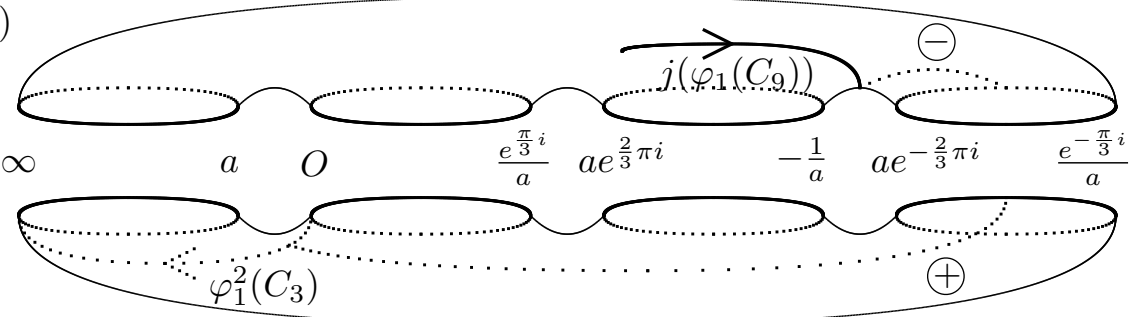

Figure A19. $\varphi_{1}^{2}\left(C_{3}\right), j\left(\varphi_{1}\left(C_{9}\right)\right)$ in $M$.

From $j\left(\varphi_{1}^{2}\left(C_{1}\right)\right)+j\left(\varphi_{1}^{2}\left(C_{7}\right)\right) \sim j\left(\varphi_{1}\left(C_{3}\right)\right)$, we have $C_{1}+C_{7} \sim j\left(\varphi_{1}^{2}\left(C_{3}\right)\right)$ (see Figure A20). 
(ii)

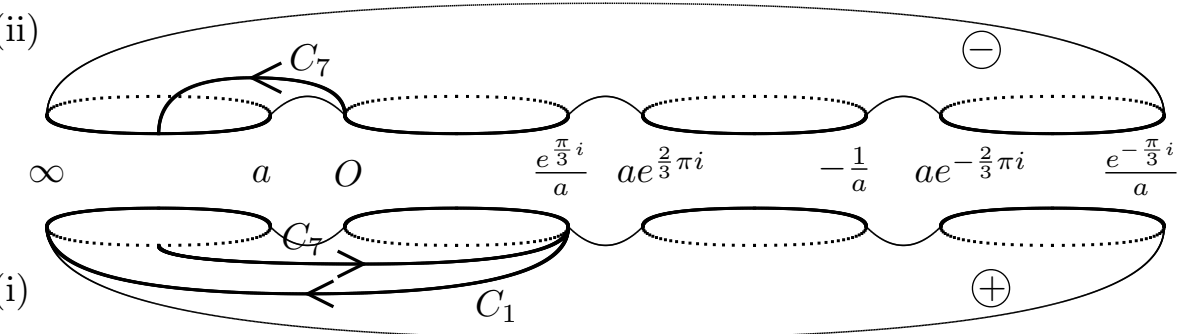

Figure A20. $C_{1}, C_{7}$ in $M$.

Therefore, we can obtain the same canonical homology basis as for $\phi \in\left(-\frac{\pi}{6}, \frac{\pi}{6}\right)$ (see Figure A13).

Finally, by Figures A15 and A20, we find $C_{1}-C_{2}+j\left(C_{7}\right)-j\left(C_{8}\right) \sim 0$. Combining (A10) and $\int_{C_{1}-C_{2}+j\left(C_{7}\right)-j\left(C_{8}\right)} \frac{1-z^{2}}{w} d z=0$ implies

$$
\int_{0}^{1} \frac{1+a^{2} t^{2}}{\sqrt{t\left(1-t^{3}\right)} \sqrt{a^{3} t^{3}+\frac{1}{a^{3}}}} d t=\frac{\sqrt{3}}{2}\left\{i \int_{0}^{1} \frac{1-a^{2} t^{2}}{\sqrt{t\left(1-t^{3}\right)} \sqrt{a^{3} t^{3}+\frac{1}{a^{3}}}} d t-\int_{0}^{1} \frac{a^{2}-t^{2}}{\sqrt{t\left(1-t^{3}\right)} \sqrt{a^{3}+\frac{t^{3}}{a^{3}}}} d t .\right.
$$

Appendix A.1.2. Periods of the Abelian Differentials of the Second Kind

Let $\left\{A_{j}, B_{j}\right\}_{j=1}^{3}$ be the canonical homology basis on $M$ which is given in the previous subsection. We first calculate the complex period matrix

$$
\left(\int_{A_{1}} G_{1} \int_{A_{2}} G_{1} \quad \int_{A_{3}} G_{1} \quad \int_{B_{1}} G_{1} \quad \int_{B_{2}} G_{1} \quad \int_{B_{3}} G_{1}\right) .
$$

Straightforward calculations yield

$$
\begin{aligned}
& \int_{C_{1}} \frac{1-z^{2}}{w} d z=-\frac{\sqrt{3}}{2 a} \int_{0}^{1} \frac{1+a^{2} t^{2}}{\sqrt{t\left(1-t^{3}\right)} \sqrt{a^{3} t^{3}+\frac{1}{a^{3}}}} d t+\frac{i}{2 a} \int_{0}^{1} \frac{1-a^{2} t^{2}}{\sqrt{t\left(1-t^{3}\right)} \sqrt{a^{3} t^{3}+\frac{1}{a^{3}}}} d t \\
& \int_{C_{1}} \frac{i\left(1+z^{2}\right)}{w} d z=\frac{1}{2 a} \int_{0}^{1} \frac{1+a^{2} t^{2}}{\sqrt{t\left(1-t^{3}\right)} \sqrt{a^{3} t^{3}+\frac{1}{a^{3}}}} d t+\frac{\sqrt{3} i}{2 a} \int_{0}^{1} \frac{1-a^{2} t^{2}}{\sqrt{t\left(1-t^{3}\right)} \sqrt{a^{3} t^{3}+\frac{1}{a^{3}}}} d t \\
& \int_{C_{1}} \frac{2 z}{w} d z=-2 i \int_{0}^{1} \frac{t}{\sqrt{t\left(1-t^{3}\right)} \sqrt{a^{3} t^{3}+\frac{1}{a^{3}}}} d t \\
& \int_{C_{2}} \frac{1-z^{2}}{w} d z=-\frac{i}{a} \int_{0}^{1} \frac{1-a^{2} t^{2}}{\sqrt{t\left(1-t^{3}\right)} \sqrt{a^{3} t^{3}+\frac{1}{a^{3}}}} d t \\
& \int_{C_{2}} \frac{i\left(1+z^{2}\right)}{w} d z=\frac{1}{a} \int_{0}^{1} \frac{1+a^{2} t^{2}}{\sqrt{t\left(1-t^{3}\right)} \sqrt{a^{3} t^{3}+\frac{1}{a^{3}}}} d t \\
& \int_{C_{2}} \frac{2 z}{w} d z=-2 i \int_{0}^{1} \frac{t}{\sqrt{t\left(1-t^{3}\right)} \sqrt{a^{3} t^{3}+\frac{1}{a^{3}}}} d t .
\end{aligned}
$$


From (A3), (A6), (A7), (A9) and (A11), we find

$$
\begin{aligned}
& \int_{C_{3}} \frac{1-z^{2}}{w} d z=2 i \int_{0}^{1} \frac{1+t^{2}}{\sqrt{t\left\{\left(\frac{1}{a^{3}}-a^{3}\right) t^{3}-i\left(1+t^{6}\right)\right\}}} d t \\
& =\frac{2}{a}\left\{-\frac{\sqrt{3}}{3} \int_{0}^{1} \frac{1+a^{2} t^{2}}{\sqrt{t\left(1-t^{3}\right)} \sqrt{a^{3} t^{3}+\frac{1}{a^{3}}}} d t+i \int_{0}^{1} \frac{1-a^{2} t^{2}}{\sqrt{t\left(1-t^{3}\right)} \sqrt{a^{3} t^{3}+\frac{1}{a^{3}}}} d t\right\}, \\
& \int_{C_{3}} \frac{i\left(1+z^{2}\right)}{w} d z=0, \quad \int_{C_{3}} \frac{2 z}{w} d z=-2 \int_{0}^{\infty} \frac{t}{\sqrt{t\left\{\left(\frac{1}{a^{3}}-a^{3}\right) t^{3}-i\left(1+t^{6}\right)\right\}}} d t .
\end{aligned}
$$

Therefore, setting

$$
\begin{aligned}
& A:=\frac{1}{a} \int_{0}^{1} \frac{1+a^{2} t^{2}}{\sqrt{t\left(1-t^{3}\right)} \sqrt{a^{3} t^{3}+\frac{1}{a^{3}}}} d t, B:=\frac{1}{a} \int_{0}^{1} \frac{1-a^{2} t^{2}}{\sqrt{t\left(1-t^{3}\right)} \sqrt{a^{3} t^{3}+\frac{1}{a^{3}}}} d t \\
& C:=4 \int_{0}^{1} \frac{t}{\sqrt{t\left(1-t^{3}\right)} \sqrt{a^{3} t^{3}+\frac{1}{a^{3}}}} d t, D:=4 \int_{0}^{\infty} \frac{t}{\sqrt{t\left\{\left(\frac{1}{a^{3}}-a^{3}\right) t^{3}-i\left(1+t^{6}\right)\right\}}} d t,
\end{aligned}
$$

we have

$$
\begin{aligned}
& \left(\int_{A_{1}} G_{1} \int_{A_{2}} G_{1} \int_{A_{3}} G_{1} \int_{B_{1}} G_{1} \int_{B_{2}} G_{1} \int_{B_{3}} G_{1}\right) \\
& =\left(\begin{array}{cccccc}
2 i B & -\frac{2}{\sqrt{3}} A-2 i B & -\frac{A}{\sqrt{3}}-i B & \frac{2}{\sqrt{3}} A & \sqrt{3} A-3 i B & \frac{2}{\sqrt{3}} A-2 i B \\
-2 A & 0 & A+\sqrt{3} i B & -2 \sqrt{3} i B & A-\sqrt{3} i B & 0 \\
i C & -2 i C+D & 2 i C-D & i C-D & 0 & -D
\end{array}\right)
\end{aligned}
$$

for $\phi \in\left(-\frac{\pi}{6}, \frac{\pi}{3}\right]$.

Next we shall calculate

$$
\left(\int_{A_{1}} G_{2} \int_{A_{2}} G_{2} \int_{A_{3}} G_{2} \quad \int_{B_{1}} G_{2} \int_{B_{2}} G_{2} \int_{B_{3}} G_{2}\right) .
$$

The following lemma appears in Section 3.2 of [8].

Lemma A1. For an arbitrary one-cycle $\gamma$, we have

$$
\begin{array}{r}
\int_{\gamma} \frac{z^{4}}{w^{3}} d z=\frac{a^{6}}{3\left(z^{6}+1\right)^{2}}\left\{2 \int_{\gamma} \frac{z^{3}}{w} d z+\frac{1-a^{6}}{a^{3}} \int_{\gamma} \frac{d z}{w}\right\}, \\
\int_{\gamma} \frac{z^{5}}{w^{3}} d z=\frac{a^{6}}{\left(z^{6}+1\right)^{2}}\left\{2 \int_{\gamma} \frac{z^{4}}{w} d z+\frac{1-a^{6}}{a^{3}} \int_{\gamma} \frac{z}{w} d z\right\}, \\
\int_{\gamma} \frac{z^{6}}{w^{3}} d z=\frac{5 a^{6}}{3\left(z^{6}+1\right)^{2}}\left\{2 \int_{\gamma} \frac{z^{5}}{w} d z+\frac{1-a^{6}}{a^{3}} \int_{\gamma} \frac{z^{2}}{w} d z\right\} .
\end{array}
$$


Note that $M$ can be constructed via a glueing the following two curves by the relation $x=-1 / z$, $y=w / z^{4}$ :

$$
w^{2}=z\left(z^{3}-a^{3}\right)\left(z^{3}+\frac{1}{a^{3}}\right) \underbrace{\longleftrightarrow}_{x=-\frac{1}{z}, y=\frac{w}{z^{4}}} y^{2}=x\left(x^{3}-a^{3}\right)\left(x^{3}+\frac{1}{a^{3}}\right) .
$$

We also have Lemma A1 in the coordinates $(x, y)$.

We now assume $\phi \in\left(-\frac{\pi}{6}, \frac{\pi}{6}\right)$. $C_{1}$ and $C_{5}$ can be rewritten as

$$
\begin{aligned}
& C_{1}=\left\{(x, y)=\left(a e^{-\frac{\pi}{3} i} t, a^{2} e^{-\frac{\pi}{6} i} \sqrt{-t\left(1+t^{3}\right)\left(-a^{3} t^{3}+1 / a^{3}\right)}\right) \mid-1 \leq t \leq 0\right\}, \\
& C_{5}=\left\{(x, y)=\left(t / a, \sqrt{-t\left(1+t^{3}\right)\left(a^{3}-t^{3} / a^{3}\right)} / a^{2}\right) \mid-1 \leq t \leq 0\right\} .
\end{aligned}
$$

Recall that $C_{1}+C_{4}-C_{2}-C_{5} \sim 0$ and $C_{1}-j\left(C_{1}\right)+C_{4}-j\left(C_{4}\right)-C_{2}+j\left(C_{2}\right)-C_{5}+j\left(C_{5}\right)$ is a closed curve on $M$. Hence we obtain

$$
\begin{aligned}
\int_{C_{1}-j\left(C_{1}\right)+C_{4}-j\left(C_{4}\right)-C_{2}+j\left(C_{2}\right)-C_{5}+j\left(C_{5}\right)} \frac{z^{4}}{w^{3}} d z \\
\quad=\int_{C_{1}-j\left(C_{1}\right)} \frac{z^{4}}{w^{3}} d z+\int_{C_{4}-j\left(C_{4}\right)} \frac{z^{4}}{w^{3}} d z-\int_{C_{2}-j\left(C_{2}\right)} \frac{z^{4}}{w^{3}} d z-\int_{C_{5}-j\left(C_{5}\right)} \frac{z^{4}}{w^{3}} d z \\
=\int_{C_{1}-j\left(C_{1}\right)} \frac{x^{6}}{y^{3}} d x+\int_{C_{4}-j\left(C_{4}\right)} \frac{z^{4}}{w^{3}} d z-\int_{C_{2}-j\left(C_{2}\right)} \frac{z^{4}}{w^{3}} d z-\int_{C_{5}-j\left(C_{5}\right)} \frac{x^{6}}{y^{3}} d x=0 .
\end{aligned}
$$

Combining this equation and Lemma A1 yields

$$
\begin{aligned}
& a^{2} e^{-\frac{\pi}{3} i} \int_{0}^{1} \frac{1-a^{6}-2 t^{3}}{\sqrt{t\left(1-t^{3}\right)} \sqrt{a^{3}+\frac{t^{3}}{a^{3}}}} d t+5 a^{2} e^{\frac{\pi}{6} i} \int_{0}^{1} \frac{-\left(1-a^{6}\right) t^{2}-2 a^{6} t^{5}}{\sqrt{t\left(1-t^{3}\right)} \sqrt{a^{3} t^{3}+\frac{1}{a^{3}}}} d t \\
& \quad+i \int_{0}^{1} \frac{1-a^{6}+2 a^{6} t^{3}}{\sqrt{t\left(1-t^{3}\right)} \sqrt{a^{3} t^{3}+\frac{1}{a^{3}}}} d t-5 \int_{0}^{1} \frac{\left(1-a^{6}\right) t^{2}-2 t^{5}}{\sqrt{t\left(1-t^{3}\right)} \sqrt{a^{3}+\frac{t^{3}}{a^{3}}}} d t=0 .
\end{aligned}
$$

Similarly, $C_{1}-C_{3}+j\left(C_{4}\right) \sim 0$ and $C_{1}-j\left(C_{1}\right)-C_{3}+j\left(C_{3}\right)-C_{4}+j\left(C_{4}\right)$ is a closed curve on $M$. By Lemma A1 and $\int_{C_{1}-j\left(C_{1}\right)-C_{3}+j\left(C_{3}\right)-C_{4}+j\left(C_{4}\right)} \frac{z^{4}}{w^{3}} d z=0$, we have

$$
\begin{aligned}
i \int_{0}^{\infty} & \frac{t^{4}}{\sqrt{t\left\{\left(\frac{1}{a^{3}}-a^{3}\right) t^{3}-i\left(1+t^{6}\right)\right\}}} d t \\
& =\frac{e^{\frac{\pi}{6}} a^{4}}{3\left(a^{6}+1\right)^{2}}\left\{i \int_{0}^{1} \frac{1-a^{6}-2 t^{3}}{\sqrt{t\left(1-t^{3}\right)} \sqrt{a^{3}+\frac{t^{3}}{a^{3}}}} d t-5 \int_{0}^{1} \frac{\left(1-a^{6}\right) t^{2}+2 a^{6} t^{5}}{\sqrt{t\left(1-t^{3}\right)} \sqrt{a^{3} t^{3}+\frac{1}{a^{3}}}} d t\right\} .
\end{aligned}
$$

From Lemma A1 and $\int_{C_{1}-j\left(C_{1}\right)-C_{3}+j\left(C_{3}\right)-C_{4}+j\left(C_{4}\right)} \frac{z^{6}}{w^{3}} d z=0$, we find

$$
\begin{aligned}
i \int_{0}^{\infty} & \frac{t^{6}}{\sqrt{t\left\{\left(\frac{1}{a^{3}}-a^{3}\right) t^{3}-i\left(1+t^{6}\right)\right\}}} d t \\
& =\frac{e^{-\frac{\pi}{6} i} a^{2}}{3\left(a^{6}+1\right)^{2}}\left\{5 i \int_{0}^{1} \frac{\left(1-a^{6}\right) t^{2}-2 t^{5}}{\sqrt{t\left(1-t^{3}\right)} \sqrt{a^{3}+\frac{t^{3}}{a^{3}}}} d t-\int_{0}^{1} \frac{1-a^{6}+2 a^{6} t^{3}}{\sqrt{t\left(1-t^{3}\right)} \sqrt{a^{3} t^{3}+\frac{1}{a^{3}}}} d t\right\} .
\end{aligned}
$$


By Lemma A1 and $\int_{C_{1}-j\left(C_{1}\right)-C_{3}+j\left(C_{3}\right)-C_{4}+j\left(C_{4}\right)} \frac{z^{5}}{w^{3}} d z=0$, we obtain

$$
\begin{aligned}
& \int_{0}^{\infty} \frac{t^{5}}{\sqrt{t\left\{\left(\frac{1}{a^{3}}-a^{3}\right) t^{3}-i\left(1+t^{6}\right)\right\}^{3}}} d t \\
& \quad=\frac{a^{3}}{\left(a^{6}+1\right)^{2}}\left\{\int_{0}^{1} \frac{\left(1-a^{6}\right) t-2 t^{4}}{\sqrt{t\left(1-t^{3}\right)} \sqrt{a^{3}+\frac{t^{3}}{a^{3}}}} d t+i \int_{0}^{1} \frac{\left(1-a^{6}\right) t+2 a^{6} t^{4}}{\sqrt{t\left(1-t^{3}\right)} \sqrt{a^{3} t^{3}+\frac{1}{a^{3}}}} d t\right\} .
\end{aligned}
$$

From $(\mathrm{A} 17)-(\mathrm{A} 18)=0$, we have

$$
\begin{aligned}
& a^{2} e^{\frac{\pi}{6}} i\left\{i \int_{0}^{1} \frac{1-a^{6}-2 t^{3}}{\sqrt{t\left(1-t^{3}\right)} \sqrt{a^{3}+\frac{t^{3}}{a^{3}}}} d t-5 \int_{0}^{1} \frac{\left(1-a^{6}\right) t^{2}+2 a^{6} t^{5}}{\sqrt{t\left(1-t^{3}\right)} \sqrt{a^{3} t^{3}+\frac{1}{a^{3}}}} d t\right\} \\
& -e^{-\frac{\pi}{6} i}\left\{-\int_{0}^{1} \frac{1-a^{6}+2 a^{6} t^{3}}{\sqrt{t\left(1-t^{3}\right)} \sqrt{a^{3} t^{3}+\frac{1}{a^{3}}}} d t+5 i \int_{0}^{1} \frac{\left(1-a^{6}\right) t^{2}-2 t^{5}}{\sqrt{t\left(1-t^{3}\right)} \sqrt{a^{3}+\frac{t^{3}}{a^{3}}}} d t\right\}=0 .
\end{aligned}
$$

Combining (A16) and (A20) yields

$$
\begin{aligned}
& \frac{a^{2}}{2} \int_{0}^{1} \frac{1-a^{6}-2 t^{3}}{\sqrt{t\left(1-t^{3}\right)} \sqrt{a^{3}+\frac{t^{3}}{a^{3}}} d t} \\
& \quad=-\frac{5}{2 \sqrt{3}} a^{2} \int_{0}^{1} \frac{\left(1-a^{6}\right) t^{2}+2 a^{6} t^{5}}{\sqrt{t\left(1-t^{3}\right)} \sqrt{a^{3} t^{3}+\frac{1}{a^{3}}}} d t+\frac{1}{\sqrt{3}} \int_{0}^{1} \frac{1-a^{6}+2 a^{6} t^{3}}{\sqrt{t\left(1-t^{3}\right)} \sqrt{a^{3} t^{3}+\frac{1}{a^{3}}}} d t \\
& 5 \int_{0}^{1} \frac{\left(1-a^{6}\right) t^{2}-2 t^{5}}{\sqrt{t\left(1-t^{3}\right)} \sqrt{a^{3}+\frac{t^{3}}{a^{3}}} d t} \\
& \quad=-\frac{10}{\sqrt{3}} a^{2} \int_{0}^{1} \frac{\left(1-a^{6}\right) t^{2}+2 a^{6} t^{5}}{\sqrt{t\left(1-t^{3}\right)} \sqrt{a^{3} t^{3}+\frac{1}{a^{3}}}} d t+\frac{1}{\sqrt{3}} \int_{0}^{1} \frac{1-a^{6}+2 a^{6} t^{3}}{\sqrt{t\left(1-t^{3}\right)} \sqrt{a^{3} t^{3}+\frac{1}{a^{3}}}} d t
\end{aligned}
$$

Next we assume $\phi \in\left[\frac{\pi}{6}, \frac{\pi}{3}\right] . C_{1}$ and $C_{8}$ can be rewritten as

$$
\begin{aligned}
& C_{1}=\left\{(x, y)=\left(a e^{-\frac{\pi}{3} i} t, a^{2} e^{-\frac{\pi}{6} i} \sqrt{-t\left(1+t^{3}\right)\left(-a^{3} t^{3}+1 / a^{3}\right)}\right) \mid-1 \leq t \leq 0\right\} \\
& C_{8}=\left\{(x, y)=\left(t / a, \sqrt{-t\left(1+t^{3}\right)\left(a^{3}-t^{3} / a^{3}\right)} / a^{2}\right) \mid-1 \leq t \leq 0\right\} .
\end{aligned}
$$

$C_{1}-C_{2}+j\left(C_{7}\right)-j\left(C_{8}\right) \sim 0$ and $C_{1}-j\left(C_{1}\right)-C_{2}+j\left(C_{2}\right)-C_{7}+j\left(C_{7}\right)+C_{8}-j\left(C_{8}\right)$ is a closed curve on $M$. From Lemma A1 and $\int_{C_{1}-j\left(C_{1}\right)-C_{2}+j\left(C_{2}\right)-C_{7}+j\left(C_{7}\right)+C_{8}-j\left(C_{8}\right)} \frac{z^{4}}{w^{3}} d z=0$, we find

$$
\begin{aligned}
& -a^{2} e^{-\frac{\pi}{3} i} \int_{0}^{1} \frac{1-a^{6}-2 t^{3}}{\sqrt{t\left(1-t^{3}\right)} \sqrt{a^{3}+\frac{t^{3}}{a^{3}}}} d t+5 a^{2} e^{\frac{\pi}{6} i} \int_{0}^{1} \frac{-\left(1-a^{6}\right) t^{2}-2 a^{6} t^{5}}{\sqrt{t\left(1-t^{3}\right)} \sqrt{a^{3} t^{3}+\frac{1}{a^{3}}}} d t \\
& +i \int_{0}^{1} \frac{1-a^{6}+2 a^{6} t^{3}}{\sqrt{t\left(1-t^{3}\right)} \sqrt{a^{3} t^{3}+\frac{1}{a^{3}}}} d t+5 \int_{0}^{1} \frac{\left(1-a^{6}\right) t^{2}-2 t^{5}}{\sqrt{t\left(1-t^{3}\right)} \sqrt{a^{3}+\frac{t^{3}}{a^{3}}}} d t=0 .
\end{aligned}
$$


$j\left(C_{2}\right)-C_{3}+j\left(C_{8}\right) \sim 0$ and $-C_{2}+j\left(C_{2}\right)-C_{3}+j\left(C_{3}\right)-C_{8}+j\left(C_{8}\right)$ is a closed curve on $M$. By Lemma A1 and $\int_{-C_{2}+j\left(C_{2}\right)-C_{3}+j\left(C_{3}\right)-C_{8}+j\left(C_{8}\right)} \frac{z^{4}}{w^{3}} d z=0$, we obtain

$$
\begin{aligned}
i \int_{0}^{\infty} & \frac{t^{4}}{\sqrt{t\left\{\left(\frac{1}{a^{3}}-a^{3}\right) t^{3}-i\left(1+t^{6}\right)\right\}^{3}}} d t \\
\quad & =\frac{a^{2}}{3\left(a^{6}+1\right)^{2}}\left\{-5 \int_{0}^{1} \frac{\left(1-a^{6}\right) t^{2}-2 t^{5}}{\sqrt{t\left(1-t^{3}\right)} \sqrt{a^{3}+\frac{t^{3}}{a^{3}}}} d t+i \int_{0}^{1} \frac{1-a^{6}+2 a^{6} t^{3}}{\sqrt{t\left(1-t^{3}\right)} \sqrt{a^{3} t^{3}+\frac{1}{a^{3}}}} d t\right\} .
\end{aligned}
$$

From Lemma A1 and $\int_{-C_{2}+j\left(C_{2}\right)-C_{3}+j\left(C_{3}\right)-C_{8}+j\left(C_{8}\right)} \frac{z^{6}}{w^{3}} d z=0$, we have

$$
\begin{aligned}
i \int_{0}^{\infty} & \frac{t^{6}}{\sqrt{t\left\{\left(\frac{1}{a^{3}}-a^{3}\right) t^{3}-i\left(1+t^{6}\right)\right\}}} d t \\
& =\frac{a^{4}}{3\left(a^{6}+1\right)^{2}}\left\{\int_{0}^{1} \frac{1-a^{6}-2 t^{3}}{\sqrt{t\left(1-t^{3}\right)} \sqrt{a^{3}+\frac{t^{3}}{a^{3}}}} d t-5 i \int_{0}^{1} \frac{\left(1-a^{6}\right) t^{2}+2 a^{6} t^{5}}{\sqrt{t\left(1-t^{3}\right)} \sqrt{a^{3} t^{3}+\frac{1}{a^{3}}}} d t\right\}
\end{aligned}
$$

By Lemma A1 and $\int_{-C_{2}+j\left(C_{2}\right)-C_{3}+j\left(C_{3}\right)-C_{8}+j\left(C_{8}\right)} \frac{z^{5}}{w^{3}} d z=0$, we find

$$
\begin{aligned}
\int_{0}^{\infty} & \frac{t^{5}}{\sqrt{t\left\{\left(\frac{1}{a^{3}}-a^{3}\right) t^{3}-i\left(1+t^{6}\right)\right\}}} d t \\
& =\frac{a^{3}}{\left(a^{6}+1\right)^{2}}\left\{\int_{0}^{1} \frac{\left(1-a^{6}\right) t-2 t^{4}}{\sqrt{t\left(1-t^{3}\right)} \sqrt{a^{3}+\frac{t^{3}}{a^{3}}}} d t-i \int_{0}^{1} \frac{\left(1-a^{6}\right) t+2 a^{6} t^{4}}{\sqrt{t\left(1-t^{3}\right)} \sqrt{a^{3} t^{3}+\frac{1}{a^{3}}}} d t\right\} .
\end{aligned}
$$

From $(\mathrm{A} 24)-(\mathrm{A} 24)=0$, we have

$$
\begin{aligned}
& -a^{2} \int_{0}^{1} \frac{1-a^{6}-2 t^{3}}{\sqrt{t\left(1-t^{3}\right)} \sqrt{a^{3}+\frac{t^{3}}{a^{3}}}} d t+5 i a^{2} \int_{0}^{1} \frac{\left(1-a^{6}\right) t^{2}+2 a^{6} t^{5}}{\sqrt{t\left(1-t^{3}\right)} \sqrt{a^{3} t^{3}+\frac{1}{a^{3}}}} d t \\
& \quad+i \int_{0}^{1} \frac{1-a^{6}+2 a^{6} t^{3}}{\sqrt{t\left(1-t^{3}\right)} \sqrt{a^{3} t^{3}+\frac{1}{a^{3}}}} d t-5 \int_{0}^{1} \frac{\left(1-a^{6}\right) t^{2}-2 t^{5}}{\sqrt{t\left(1-t^{3}\right)} \sqrt{a^{3}+\frac{t^{3}}{a^{3}}}} d t=0 .
\end{aligned}
$$

Combining (A23) and (A27) yields

$$
\begin{aligned}
& \frac{a^{2}}{2} \int_{0}^{1} \frac{1-a^{6}-2 t^{3}}{\sqrt{t\left(1-t^{3}\right)} \sqrt{a^{3}+\frac{t^{3}}{a^{3}}}} d t \\
& \quad=-\frac{5}{2 \sqrt{3}} a^{2} \int_{0}^{1} \frac{\left(1-a^{6}\right) t^{2}+2 a^{6} t^{5}}{\sqrt{t\left(1-t^{3}\right)} \sqrt{a^{3} t^{3}+\frac{1}{a^{3}}}} d t+\frac{1}{\sqrt{3}} e^{\frac{2}{3} \pi i} \int_{0}^{1} \frac{1-a^{6}+2 a^{6} t^{3}}{\sqrt{t\left(1-t^{3}\right)} \sqrt{a^{3} t^{3}+\frac{1}{a^{3}}}} d t \\
& 5 \int_{0}^{1} \frac{\left(1-a^{6}\right) t^{2}-2 t^{5}}{\sqrt{t\left(1-t^{3}\right)} \sqrt{a^{3}+\frac{t^{3}}{a^{3}}}} d t \\
& \quad=\frac{10}{\sqrt{3}} e^{\frac{\pi}{3} i} a^{2} \int_{0}^{1} \frac{\left(1-a^{6}\right) t^{2}+2 a^{6} t^{5}}{\sqrt{t\left(1-t^{3}\right)} \sqrt{a^{3} t^{3}+\frac{1}{a^{3}}}} d t+\frac{1}{\sqrt{3}} \int_{0}^{1} \frac{1-a^{6}+2 a^{6} t^{3}}{\sqrt{t\left(1-t^{3}\right)} \sqrt{a^{3} t^{3}+\frac{1}{a^{3}}}} d t
\end{aligned}
$$


For $\phi \in\left(-\frac{\pi}{6}, \frac{\pi}{3}\right]$, straightforward calculations yield

$$
\begin{aligned}
& \int_{C_{1}-j\left(C_{1}\right)} \frac{z^{4}}{w^{3}} d z=-\frac{10 e^{\frac{\pi}{6} i} a^{4}}{3\left(a^{6}+1\right)^{2}} \int_{0}^{1} \frac{\left(1-a^{6}\right) t^{2}+2 a^{6} t^{5}}{\sqrt{t\left(1-t^{3}\right)} \sqrt{a^{3} t^{3}+\frac{1}{a^{3}}}} d t \\
& \int_{C_{1}-j\left(C_{1}\right)} \frac{z^{5}}{w^{3}} d z=-\frac{2 i a^{3}}{\left(a^{6}+1\right)^{2}} \int_{0}^{1} \frac{\left(1-a^{6}\right) t+2 a^{6} t^{4}}{\sqrt{t\left(1-t^{3}\right)} \sqrt{a^{3} t^{3}+\frac{1}{a^{3}}}} d t \\
& \int_{C_{1}-j\left(C_{1}\right)} \frac{z^{6}}{w^{3}} d z=\frac{2 e^{-\frac{\pi}{6} i} a^{2}}{3\left(a^{6}+1\right)^{2}} \int_{0}^{1} \frac{1-a^{6}+2 a^{6} t^{3}}{\sqrt{t\left(1-t^{3}\right)} \sqrt{a^{3} t^{3}+\frac{1}{a^{3}}}} d t \\
& \int_{C_{2}-j\left(C_{2}\right)} \frac{z^{4}}{w^{3}} d z=-\frac{2 i a^{2}}{3\left(a^{6}+1\right)^{2}} \int_{0}^{1} \frac{1-a^{6}+2 a^{6} t^{3}}{\sqrt{t\left(1-t^{3}\right)} \sqrt{a^{3} t^{3}+\frac{1}{a^{3}}}} d t \\
& \int_{C_{2}-j\left(C_{2}\right)} \frac{z^{5}}{w^{3}} d z=-\frac{2 i a^{3}}{\left(a^{6}+1\right)^{2}} \int_{0}^{1} \frac{\left(1-a^{6}\right) t+2 a^{6} t^{4}}{\sqrt{t\left(1-t^{3}\right)} \sqrt{a^{3} t^{3}+\frac{1}{a^{3}}}} d t \\
& \int_{C_{2}-j\left(C_{2}\right)} \frac{z^{6}}{w^{3}} d z=-\frac{10 i a^{4}}{3\left(a^{6}+1\right)^{2}} \int_{0}^{1} \frac{\left(1-a^{6}\right) t^{2}+2 a^{6} t^{5}}{\sqrt{t\left(1-t^{3}\right)} \sqrt{a^{3} t^{3}+\frac{1}{a^{3}}}} d t .
\end{aligned}
$$

Moreover, by (A17)-(A19), (A21), (A22), (A24)-(A26), (A28) and (A29), we find

$$
\begin{aligned}
& \int_{C_{3}} \frac{z^{4}}{w^{3}} d z=-\int_{C_{3}} \frac{z^{6}}{w^{3}} d z \\
& \quad=\frac{2 e^{\frac{\pi}{3} i} a^{2}}{3 \sqrt{3}\left(a^{6}+1\right)^{2}}\left\{-5 a^{2} \int_{0}^{1} \frac{\left(1-a^{6}\right) t^{2}+2 a^{6} t^{5}}{\sqrt{t\left(1-t^{3}\right)} \sqrt{a^{3} t^{3}+\frac{1}{a^{3}}}} d t+e^{\frac{\pi}{3} i} \int_{0}^{1} \frac{1-a^{6}+2 a^{6} t^{3}}{\sqrt{t\left(1-t^{3}\right)} \sqrt{a^{3} t^{3}+\frac{1}{a^{3}}}} d t\right\} \\
& \int_{C_{3}} \frac{z^{5}}{w^{3}} d z=-\int_{0}^{\infty} \frac{t^{5}}{\sqrt{t\left\{\left(\frac{1}{a^{3}}-a^{3}\right) t^{3}-i\left(1+t^{6}\right)\right.}{ }^{3}} d t
\end{aligned}
$$

for $\phi \in\left(-\frac{\pi}{6}, \frac{\pi}{3}\right]$.

Therefore, setting

$$
\begin{aligned}
& E:=\frac{10 a^{4}}{3\left(a^{6}+1\right)^{2}} \int_{0}^{1} \frac{\left(1-a^{6}\right) t^{2}+2 a^{6} t^{5}}{\sqrt{t\left(1-t^{3}\right)} \sqrt{a^{3} t^{3}+\frac{1}{a^{3}}}} d t, F:=\frac{2 a^{2}}{3\left(a^{6}+1\right)^{2}} \int_{0}^{1} \frac{1-a^{6}+2 a^{6} t^{3}}{\sqrt{t\left(1-t^{3}\right)} \sqrt{a^{3} t^{3}+\frac{1}{a^{3}}}} d t, \\
& H:=\frac{2 a^{3}}{\left(a^{6}+1\right)^{2}} \int_{0}^{1} \frac{\left(1-a^{6}\right) t+2 a^{6} t^{4}}{\sqrt{t\left(1-t^{3}\right)} \sqrt{a^{3} t^{3}+\frac{1}{a^{3}}}} d t, I:=2 \int_{0}^{\infty} \frac{t^{5}}{\sqrt{t\left\{\left(\frac{1}{a^{3}}-a^{3}\right) t^{3}-i\left(1+t^{6}\right)\right\}}} d t
\end{aligned}
$$

we have

$$
\begin{aligned}
& \left(\begin{array}{lll}
\int_{A_{1}} G_{2} & \int_{A_{2}} G_{2} & \int_{A_{3}} G_{2}
\end{array}\right)=\left(\begin{array}{ccc}
i(-E+F) & \frac{2}{\sqrt{3}}\left(e^{\frac{2}{3} \pi i} E-e^{\frac{\pi}{3} i} F\right) & \frac{e^{\frac{2}{3} \pi i} E-e^{\frac{\pi}{3} i} F}{\sqrt{3}} \\
-E-F & 0 & e^{-\frac{\pi}{3} i} E+e^{\frac{\pi}{3} i} F \\
i H & -2 i H+I & 2 i H-I
\end{array}\right), \\
& \left(\begin{array}{cccc}
\int_{B_{1}} G_{2} & \int_{B_{2}} G_{2} & \int_{B_{3}} G_{2}
\end{array}\right)=\left(\begin{array}{ccc}
\frac{E+F}{\sqrt{3}} & \sqrt{3}\left(e^{\frac{\pi}{3} i} E+e^{-\frac{\pi}{3} i} F\right) & \frac{2}{\sqrt{3}}\left(e^{\frac{\pi}{3} i} E+e^{-\frac{\pi}{3} i} F\right) \\
\sqrt{3} i(E-F) & e^{\frac{\pi}{3} i} E+e^{-\frac{\pi}{3} i} F & 0 \\
i H-I & 0 & -I
\end{array}\right)
\end{aligned}
$$

for $\phi \in\left(-\frac{\pi}{6}, \frac{\pi}{3}\right]$. 


\section{Appendix A.2. $t$ G Family}

Set $a=b^{4}+1 / b^{4}$, where $b=s e^{i \phi}\left(0<s<1,|\phi|<\frac{\pi}{4}\right)$. Let $M$ be a hyperelliptic Riemann surface of genus three defined by $w^{2}=z^{8}+a z^{4}+1=\left(z^{4}+b^{4}\right)\left(z^{4}+\frac{1}{b^{4}}\right)$.

Define $G_{1}:={ }^{t}\left(\frac{1-z^{2}}{w} d z, \frac{i\left(1+z^{2}\right)}{w} d z, \frac{2 z}{w} d z\right)$ as a column vector which consists of a basis of holomorphic differentials on $M$. Up to exact one-forms, the Abelian differentials of the second kind are given by

$$
\left\{\frac{1-z^{2}}{w} d z, \frac{i\left(1+z^{2}\right)}{w} d z, \frac{2 z}{w} d z, \frac{z^{4}-z^{6}}{w^{3}} d z, \frac{i\left(z^{4}+z^{6}\right)}{w^{3}} d z, \frac{z^{5}}{w^{3}} d z\right\}
$$

and we set $G_{2}={ }^{t}\left(\frac{z^{4}-z^{6}}{w^{3}} d z, \frac{i\left(z^{4}+z^{6}\right)}{w^{3}} d z, \frac{z^{5}}{w^{3}} d z\right)$. Let $\left\{A_{j}, B_{j}\right\}_{j=1}^{3}$ be a canonical homology basis on $M$. We shall determine a complex $6 \times 6$ matrix given by

$$
\left(\int_{A_{1}}\left(\begin{array}{l}
G_{1} \\
G_{2}
\end{array}\right) \int_{A_{2}}\left(\begin{array}{l}
G_{1} \\
G_{2}
\end{array}\right) \int_{A_{3}}\left(\begin{array}{l}
G_{1} \\
G_{2}
\end{array}\right) \int_{B_{1}}\left(\begin{array}{l}
G_{1} \\
G_{2}
\end{array}\right) \int_{B_{2}}\left(\begin{array}{l}
G_{1} \\
G_{2}
\end{array}\right) \int_{B_{3}}\left(\begin{array}{l}
G_{1} \\
G_{2}
\end{array}\right)\right) .
$$

Consider the following isometries on $M$ :

$$
j(z, w)=(z,-w), \varphi(z, w)=(i z, w) .
$$

It is straightforward to show that

$$
j^{*}\left(\begin{array}{l}
G_{1} \\
G_{2}
\end{array}\right)=-\left(\begin{array}{l}
G_{1} \\
G_{2}
\end{array}\right), \varphi^{*}\left(\begin{array}{l}
G_{1} \\
G_{2}
\end{array}\right)=\left(\begin{array}{cccccc}
0 & 1 & 0 & 0 & 0 & 0 \\
-1 & 0 & 0 & 0 & 0 & 0 \\
0 & 0 & -1 & 0 & 0 & 0 \\
0 & 0 & 0 & 0 & 1 & 0 \\
0 & 0 & 0 & -1 & 0 & 0 \\
0 & 0 & 0 & 0 & 0 & -1
\end{array}\right)\left(\begin{array}{l}
G_{1} \\
G_{2}
\end{array}\right) .
$$

Remark A5. For $\phi=0$, there exists an isometry defined by $\psi(z, w)=(\bar{z}, \bar{w})$ and we find

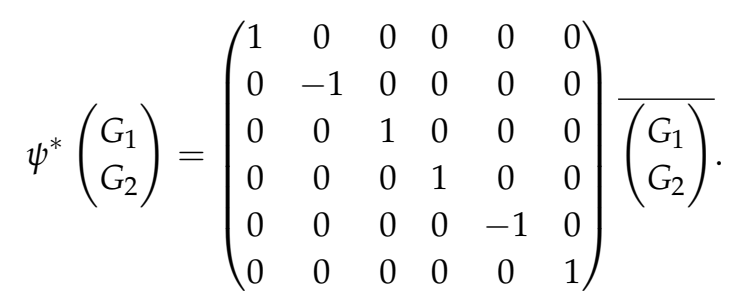

We now construct $M$ as a two-sheeted branched cover of $\overline{\mathbb{C}}$. Let $\pi: M \rightarrow \overline{\mathbb{C}}$ be the two-sheeted covering defined by $(z, w) \mapsto z$ which is branched at the following eight fixed points of $j$ :

$$
\left\{\left(b e^{ \pm \frac{\pi}{4} i}, 0\right),\left(b e^{ \pm \frac{3}{4} \pi i}, 0\right),\left(\frac{e^{ \pm \frac{\pi}{4} i}}{b}, 0\right),\left(\frac{e^{ \pm \frac{3}{4} \pi i}}{b}, 0\right)\right\} .
$$

We prepare two copies of $\overline{\mathbb{C}}$ and take two closed curves passing through the eight points, respectively. So we can divide $\overline{\mathbb{C}}$ into two domains and label " + " and " - " (see Figure A21). Slit them along the thick lines. Identifying each of the upper (resp. lower) edges of the thick lines in (i) with each of the lower (resp. upper) edges of the thick lines in (ii), we obtain the hyperelliptic Riemann surface $M$ of genus three. 
(i)

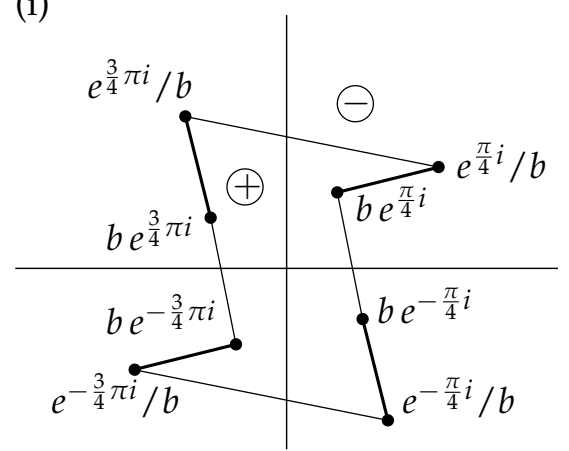

(ii)

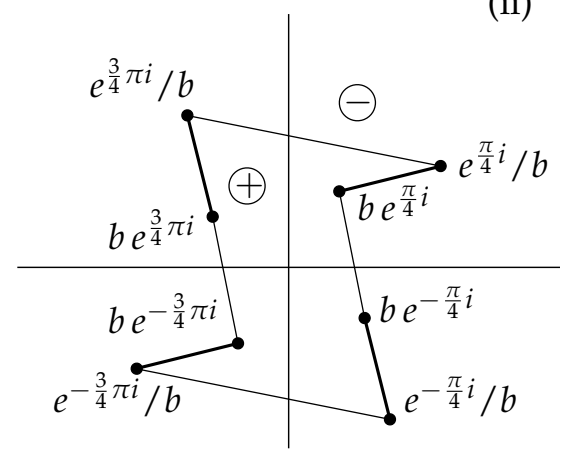

(ii)

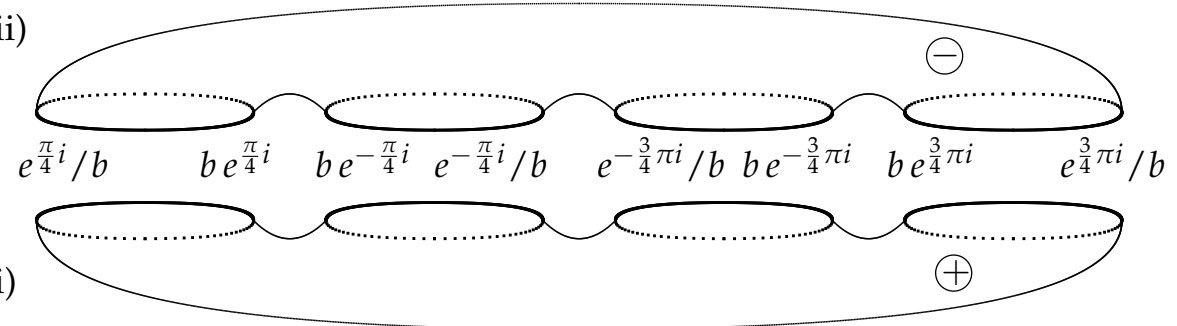

Figure A21. $M$ represented as a two-sheeted branched cover of $\overline{\mathbb{C}}$.

We can apply the same arguments as in [8] (see $§ 5.3$ in [8]). We first introduce the following key one-cycles.

$$
\begin{aligned}
C_{1}=\{(z, w) & \left.=\left(t, \sqrt{t^{8}+a t^{4}+1}\right) \mid 0 \leq t \leq \infty\right\} \\
\cup & \left\{(z, w)=\left(-i t, \sqrt{t^{8}+a t^{4}+1}\right) \mid-\infty \leq t \leq 0\right\} \\
C_{2}=\{(z, w) & \left.=\left(-i t, \sqrt{t^{8}+a t^{4}+1}\right) \mid-1 \leq t \leq 1\right\} \\
\cup & \left\{(z, w)=\left(e^{i t},-e^{2 i t} \sqrt{a+2 \cos (4 t)}\right) \mid-\pi / 2 \leq t \leq \pi / 2\right\},
\end{aligned}
$$

where we choose the branches as follows: $\arg \sqrt{t^{8}+a t^{4}+1} \in\left(-\frac{\pi}{2}, \frac{\pi}{2}\right)$ for $C_{1}, \arg \sqrt{t^{8}+a t^{4}+1} \in\left(-\frac{\pi}{2}, \frac{\pi}{2}\right)$, and $\arg \sqrt{a+2 \cos (4 t)} \in\left(-\frac{\pi}{2}, \frac{\pi}{2}\right)$ for $C_{2}$.

Remark A6. Straightforward calculations yield

$$
\begin{aligned}
t^{8}+a t^{4}+1 & =t^{8}+\left(b^{4}+\frac{1}{b^{4}}\right) t^{4}+1 \\
& =t^{8}+\left(s^{4}+\frac{1}{s^{4}}\right) t^{4} \cos (4 \phi)+1+i\left(-\frac{1}{s^{4}}+s^{4}\right) t^{4} \sin (4 \phi), \\
a+2 \cos (4 t) & =\left(s^{4}+\frac{1}{s^{4}}\right) \cos (4 \phi)+2 \cos (4 t)+i\left(-\frac{1}{s^{4}}+s^{4}\right) \sin (4 \phi) .
\end{aligned}
$$

$|\phi|<\frac{\pi}{4}$ implies $\arg \left(t^{8}+a t^{4}+1\right), \arg (a+2 \cos (4 t)) \in(-\pi, \pi)$. So we may choose the branches such that $\arg \sqrt{t^{8}+a t^{4}+1}, \arg \sqrt{a+2 \cos (4 t)} \in\left(-\frac{\pi}{2}, \frac{\pi}{2}\right)$.

A canonical homology basis on $M$ is given as follows.

$$
\begin{array}{ll}
A_{1}=C_{2}, & A_{2}=-\varphi^{2}\left(C_{1}\right)-\varphi^{3}\left(C_{1}\right)+\varphi^{3}\left(C_{2}\right), \quad A_{3}=\varphi^{2}\left(C_{2}\right), \\
B_{1}=C_{1}, & B_{2}=B_{1}+\varphi^{3}\left(C_{1}\right), \quad B_{3}=B_{2}+\varphi^{2}\left(C_{1}\right) .
\end{array}
$$


Set

$$
\begin{aligned}
& A=2 \int_{0}^{1} \frac{1-t^{2}}{\sqrt{t^{8}+a t^{4}+1}} d t+4 \int_{0}^{1} \frac{d t}{\sqrt{16 t^{4}-16 t^{2}+2+a}} \\
& B=2 \int_{0}^{1} \frac{1+t^{2}}{{\sqrt{t^{8}+a t^{4}+1}}^{1}} d t, \quad C=8 \int_{0}^{1} \frac{t}{\sqrt{t^{8}+a t^{4}+1}} d t \\
& D=8 \int_{0}^{1} \frac{d t}{\sqrt{(2+a) t^{4}+(2 a-12) t^{2}+2+a}} \\
& E=2 \int_{0}^{1} \frac{t^{4}-t^{6}}{{\sqrt{t^{8}+a t^{4}+1^{3}}}^{3}} d t+4 \int_{0}^{1} \frac{d t}{{\sqrt{16 t^{4}-16 t^{2}+2+a}}^{3}} \\
& F=2 \int_{0}^{1} \frac{t^{4}+t^{6}}{{\sqrt{t^{8}+a t^{4}+1^{3}}}^{3}} d t, \quad H=4 \int_{0}^{1} \frac{t^{5}}{{\sqrt{t^{8}+a t^{4}+1}}^{3}} d t \\
& I=4 \int_{0}^{1} \frac{\left(1+t^{2}\right)^{2}}{{\sqrt{(2+a) t^{4}+(2 a-12) t^{2}+2+a}}^{3}} d t
\end{aligned}
$$

where we choose the branches as follows:

$$
\arg \sqrt{16 t^{4}-16 t^{2}+2+a} \in\left(-\frac{\pi}{2}, \frac{\pi}{2}\right), \quad \arg \sqrt{(2+a) t^{4}+(2 a-12) t^{2}+2+a} \in\left(-\frac{\pi}{2}, \frac{\pi}{2}\right) .
$$

Remark A7. Straightforward calculations yield

$$
\begin{aligned}
& 16 t^{4}-16 t^{2}+2+a=16 t^{4}-16 t^{2}+2+b^{4}+\frac{1}{b^{4}} \\
& \quad=16 t^{4}-16 t^{2}+2+\left(s^{4}+\frac{1}{s^{4}}\right) \cos (4 \phi)+i\left(-\frac{1}{s^{4}}+s^{4}\right) \sin (4 \phi), \\
& (2+a) t^{4}+(2 a-12) t^{2}+2+a=2 t^{4}-12 t^{2}+2+a\left(t^{4}+2 t^{2}+1\right) \\
& =2 t^{4}-12 t^{2}+2+\left(s^{4}+\frac{1}{s^{4}}\right) \cos (4 \phi)\left(t^{2}+1\right)^{2}+i\left(-\frac{1}{s^{4}}+s^{4}\right)\left(t^{2}+1\right)^{2} \sin (4 \phi) .
\end{aligned}
$$

$|\phi|<\frac{\pi}{4}$ implies $\arg \left(16 t^{4}-16 t^{2}+2+a\right), \arg \left((2+a) t^{4}+(2 a-12) t^{2}+2+a\right) \in(-\pi, \pi)$. So we may choose the branches such that $\arg \sqrt{16 t^{4}-16 t^{2}+2+a}, \arg \sqrt{(2+a) t^{4}+(2 a-12) t^{2}+2+a} \in\left(-\frac{\pi}{2}, \frac{\pi}{2}\right)$.

Then

$$
\left(\int_{A_{1}}\left(\begin{array}{l}
G_{1} \\
G_{2}
\end{array}\right) \quad \int_{A_{2}}\left(\begin{array}{l}
G_{1} \\
G_{2}
\end{array}\right) \quad \int_{A_{3}}\left(\begin{array}{l}
G_{1} \\
G_{2}
\end{array}\right) \int_{B_{1}}\left(\begin{array}{l}
G_{1} \\
G_{2}
\end{array}\right) \quad \int_{B_{2}}\left(\begin{array}{l}
G_{1} \\
G_{2}
\end{array}\right) \int_{B_{3}}\left(\begin{array}{l}
G_{1} \\
G_{2}
\end{array}\right)\right)
$$

is obtained by

$$
\left(\begin{array}{cccccc}
-i B & -A & i B & -i B & -2 i B & -i B \\
A & i B & -A & i B & 0 & -i B \\
-i D & i D & -i D & C & 0 & C \\
-i F & -E & i F & -i F & -2 i F & -i F \\
E & i F & -E & i F & 0 & -i F \\
-i I & i I & -i I & H & 0 & H
\end{array}\right)
$$




\section{References}

1. Schoen, A. Infinite Periodic Minimal Surfaces without Self-Intersections; National Aeronautics and Space Administration: Washington, DC, USA, 1970.

2. Fogden, A.; Haeberlein, M.; Lidin, S. Generalizations of the gyroid surface. J. Phys. 1993, 3, 2371-2385. [CrossRef]

3. Fogden, A.; Hyde, S.T.; Continuous transformations of cubic minimal surfaces. Eur. Phys. J. Condens. Matter Complex Syst. 1999, 7, 91-104. [CrossRef]

4. Ros, A. One-sided complete stable minimal surfaces. J. Differ. Geom. 2006, 74, 69-92. [CrossRef]

5. Ejiri, N. A differential-geometric Schottky problem, and minimal surfaces in tori. Contemp. Math. 2002, 308, 101-144. [CrossRef]

6. Ejiri, N. A Generating Function of a Complex Lagrangian Cone in $\mathbb{H}^{n}$, to Appear in Comm. Anal. Geom. Available online: https://kaken.nii.ac.jp/grant/KAKENHI-PROJECT-22540103/ (accessed on 23 September 2020).

7. Ejiri, N.; Shoda, T. On a moduli theory of minimal surfaces. In Prospects of Differential Geometry and Its Related Fields; World Sci. Publ.: River Edge, NJ, USA, 2014; pp. 155-172.

8. Ejiri, N.; Shoda, T. The Morse index of a triply periodic minimal surface. Differ. Geom. Appl. 2018, 58, $177-201$. [CrossRef]

9. Mathematica; Version 11.3; Wolfram Research, Inc.: Champaign, IL, USA, 2018.

10. Meeks, W.H., III. The theory of triply periodic minimal surfaces. Indiana Univ. Math. J. 1990, 39, 877-936. [CrossRef]

11. Große-Brauckmann, K.; Wohlgemuth, M. The gyroid is embedded and has constant mean curvature companions. Calc. Var. Partial. Differ. Equ. 1996, 4, 499-523. [CrossRef]

12. Schröder-Turk, G.E.; Fogden, A.; Hyde, S.T. Bicontinuous geometries and molecular self-assembly: Comparison of local curvature and global packing variations in genus-three cubic, tetragonal and rhombohedral surfaces. Eur. Phys. J. Condens. Matter Complex Syst. 2006, 54, 509-524. [CrossRef]

(C) 2020 by the authors. Licensee MDPI, Basel, Switzerland. This article is an open access article distributed under the terms and conditions of the Creative Commons Attribution (CC BY) license (http:/ / creativecommons.org/licenses/by/4.0/). 